\title{
Review on sorption materials and technologies for heat pumps and thermal energy storage
}



\section{Abstract}

Sorption is used for absorption/adsorption heat pumps (sorption refrigeration) and sorption for thermal energy storage (TES). This paper is the first review where the research on both applications is shown together. Sorption has advanced very much due to the immense amount of research carried out around heat pumping and solar refrigeration. Moreover, sorption and thermochemical heat storage attracted considerable attention recently since this technology offers various opportunities in the design of renewable and sustainable energy systems. The paper presents the operation principle of the technology and the materials used or in research are listed and compared. Absorption heat pumping and refrigeration research is today more focussed in the decrease of unit costs and increase of energy efficiency, adsorption is focussed in finding more efficient working pairs, and storage is testing the first prototypes and designing new ones with different or enhanced storage materials and new reactor concepts to optimize energy output.

Keywords: sorption; absorption; adsorption; materials; technologies; heat pumps; thermal energy storage

\section{Nomenclature}

COP: Coefficient of Performance

SCP: specific cooling power

TES: Thermal energy storage

Qchar: heat of charging

Qsens: sensible heat

Qcond: heat of condensation

Qbind: heat of binding

HX: heat exchanger

SHX: solution heat exchanger 
PTX: Pressure-Temperature concentrationPCPs: porous coordination polymers

MOFS: metal organic frameworks

TCM: Thermochemical material

DHW: domestic hot water

HTDU: high-temperature discharging unit

LTDU: low-temperature discharging unit

T: temperature $\left[{ }^{\circ} \mathrm{C}\right]$

AlPOs: aluminophosphates

SAPOs: silico-aluminophosphates

Subscripts

$\mathrm{h}$ : heat

Greek letters

\section{Introduction}

Sorption is a technology used for absorption/adsorption heat pumps (sorption refrigeration) and sorption for thermal energy storage (TES). Although both technologies for heat pumps and sorption for TES use the same or very similar materials and technologies, they have been rarely considered together to learn from one to the other. This review aims on contributing on this collaborative learning by presenting for the first time the materials and technologies used in sorption systems for cooling, heat pumping and storage.

The existing systems for producing cold, especially when using solar thermal energy, are based mainly on the phenomena of sorption: the process by absorption liquid-gas and the process by adsorption solid-gas [1-3]. The adsorption process concerns separation of a substance from one phase, accompanied by its accumulation or concentration on the surface of another. On the other hand, absorption is the process in which material transferred from one phase to another, (e.g. liquid) interpenetrates the second phase to form a solution. In general, the main differences between absorption and adsorption are located in the nature of the sorbent and the duration of the sorption cycle, which is significantly longer for adsorption [4]; therefore the main difference is that in absorption the sorbent is a pumplable fluid, therefore the heat is recovered easier but movable parts can be a disadvantage. This kind of cold storage system, which can be driven by electricity, industry waste heat, or solar energy, contributes significantly to the concept of sustainable system development. 
73 The key figure describing the efficiency of a sorption refrigeration system is the thermal coefficient of performance $\left(\mathrm{COP}_{\text {thermal }}\right)$, which is defined as follows $[4,5]$ :

$$
C O P_{\text {thermal }}=\frac{\text { cooling power }}{\text { energy received by system }} \quad \text { Eq. } 1
$$

77

For sorption thermal storage systems, the energy for charging includes sensible heat, a prerequisite energy to heat up the reactor to a required desorption temperature, and the heat of desorption, which includes the heat of condensation and the heat of binding [6]:

$$
\begin{array}{ll}
Q_{\text {char }}=Q_{\text {sens }}+Q_{\text {des }} & \text { Eq. } 2 \\
Q_{\text {des }}=Q_{\text {cond }}+Q_{\text {bind }} & \text { Eq. } 3
\end{array}
$$

For cold storage, the value of Qcond/Qchar almost equals the cooling coefficient of performance (COPc) [6].

\section{Sorption heat pump and cooling}

\subsection{Technologies}

Adsorption cycles for heat pumping or cooling/refrigeration might seem like a relatively new, but the history of solid sorption systems is long dating from Faraday (1823) [7-9]. Patents on refrigeration machines started in early 1900s, being the first one that from Dunsford (1915) for ammonium nitrate as adsorbent for marine applications (Figure 1) [10]. The technology restarted to rise interest again in the early 1970s, with pioneer work by Alefeld (1975) [11] and Tchernev (1977) [12]. In the late 1980s and 1990s there was an explosion of activity worldwide, with conferences, published papers and the first products appearing in the market. Very recently the environmental and energy cost concerns have led to new research and development of small scale products for air conditioning, solar air conditioning, and heat pumping. Interest in storage is the most recent studied application. 


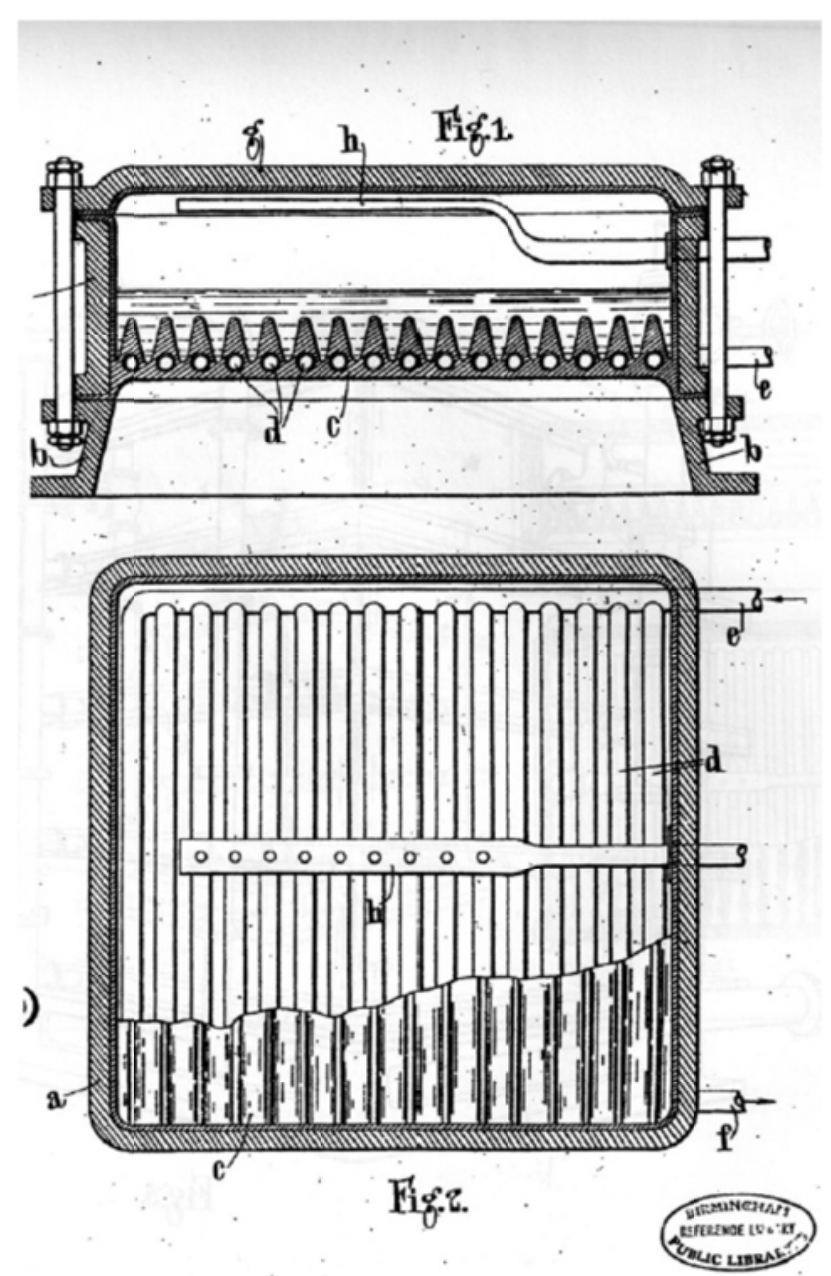

Figure 1. Dunsford's generator of $1915[7,10]$

Sorption systems are either open or closed cycles [2-5,13-16]. Open cycles are mainly desiccant systems, while closed cycles are adsorption or absorption systems. The main differences between adsorption and absorption are located in the nature of the sorbent and the duration of the sorption cycle. The general operating principal of solar closed cycle sorption refrigerator is presented in Figure 2. The process of adsorption concerns separation of a substance from one phase and its concentration on the surface of another. Adsorption systems are based on a physical or chemical reaction process in which the molecules of one substance are adsorbed on the internal surface of another substance [4,17]. 


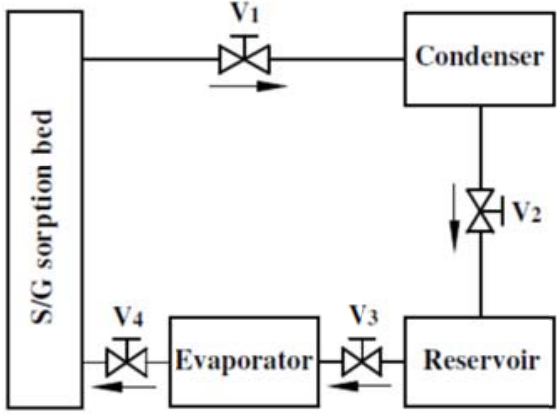

(a) Basic sorption system

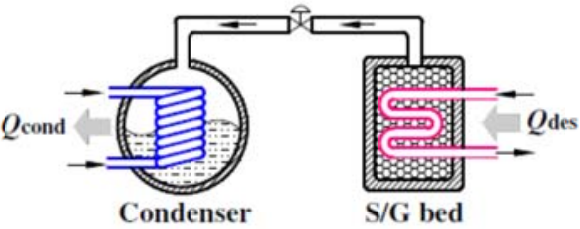

Heating desorption phase

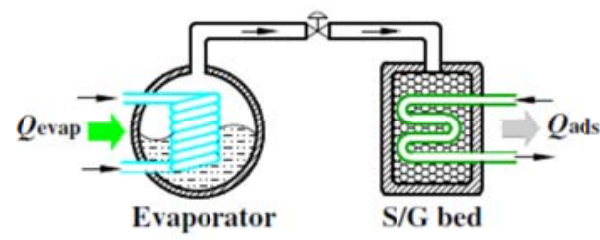

Cooling adsorption phase

(b) Working processes

Figure 2. Schematic diagram of a basic solid-gas sorption refrigeration cycle [18].

124 Absorption systems are the oldest and most common heat driven refrigeration systems $[4,16,19,20]$. On the low-pressure side, an evaporative refrigerant is absorbed by the absorbent formulating a weak absorbent solution. The weak solution is directed to the generator where the pressurisation takes place by desorption of the refrigerant. The refrigerant then undergoes a common cooling cycle, while the weak solution is directed to the absorber and the cycle is repeated.

Ziegler [13] carried out a comparison between liquid (absorption) and solid (adsorption) sorption (Figure 3), claiming that sorption chillers can be operated with low driving temperature, but the specific costs will be high and the COP will be low. Such comparison is done based on the characteristic temperature difference, $\Delta \Delta \mathrm{T}$, defined as:

134 where $t_{G}$ is the temperature of the generator (desorber), $t_{A}$ is the temperature of the absorber, $t_{C}$ is the temperature of the condenser, $\mathrm{t}_{\mathrm{E}}$ is the temperature of the evaporator, and $\mathrm{B}$ is the Düring coefficient.

Demir at al. [21] and Sarbu and Sebarcievici [5] compared the different characteristics of both systems (Table 1). 

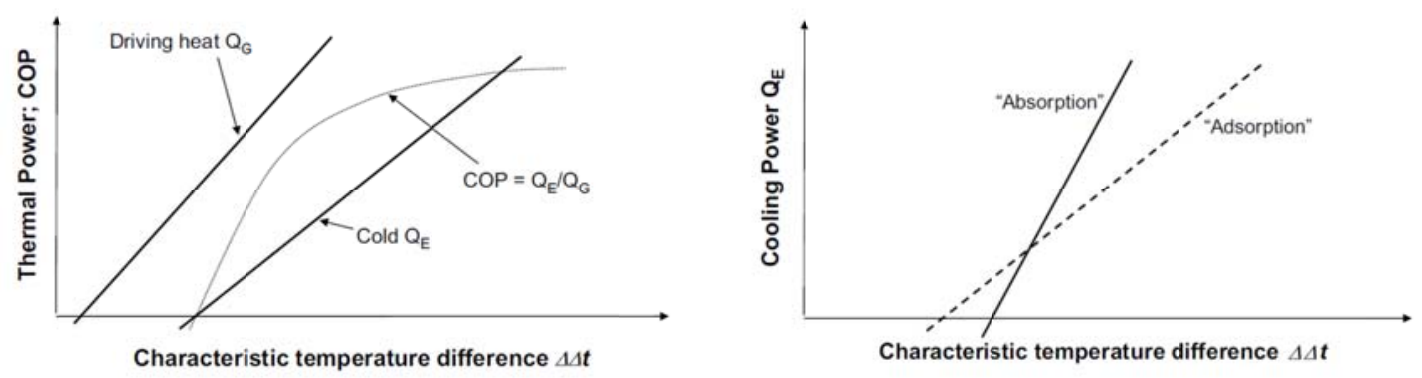

Figure 3. Thermal power (COP) and cooling power comparison between absorption and adsorption cycles

[13].

Table 1. Comparison between absorption and adsorption systems [5,21].

\begin{tabular}{|l|l|c|}
\hline \multicolumn{1}{|c|}{ Types of heat pumps } & \multicolumn{1}{|c|}{ Working pairs } & Coefficient of performance \\
\hline Adsorption & Carbon/methanol & $0.12-1.06$ \\
\cline { 2 - 3 } & Zeolite/water & $0.28-1.4$ \\
\cline { 2 - 3 } & Silica gel/water & $0.25-0.65$ \\
\hline Absorption & Methano1/water & $0.7-1.1$ \\
\cline { 2 - 3 } & Lithium bromide/water & \multicolumn{2}{|c|}{$3-4$} \\
\hline Vapour compression & & \\
\hline
\end{tabular}

143

149

150

The sorption refrigerator includes one or several reactors, i.e. regenerator(s), absorber(s), adsorber(s), generator(s) etc., depending on the specific cycle and the sorption pair, and of course the condenser and the evaporator, which exchange refrigerant vapour with the thermal compressor. The working principle of solid physisorption is based on the Van der Waals force between a sorbent and a refrigerant gas. The basic adsorption cycle for cooling consists of four processes represented in Figure 4 [20,22-26].

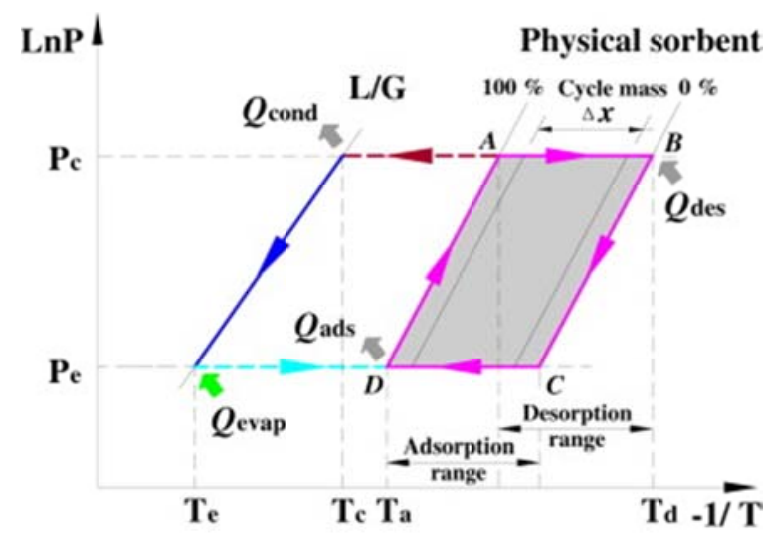

(a)

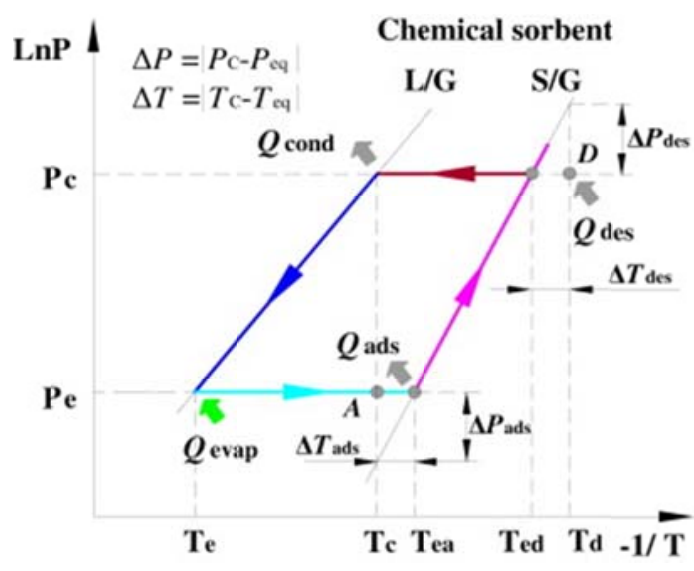

(b)

Figure 4.(a) Working principle of the physiosorption (adsorption) refrigeration cycle; (b) Working principle of 
The working principle of solid thermochemical sorption refrigeration cycle is shown in Figure $4 \mathrm{~b}$ [22]. The working pressure and temperature are the functional equation of one variable, and the working region between the sorbent and the refrigerant is the solid-gasi equilibrium line. Thus, once the operating temperature/pressure is away from the solid-gas equilibrium temperature/pressure, chemical reaction would occur between the sorbent and the refrigerant. The driving equilibrium drop is the only contributor to the chemical reaction in solid-gas thermochemical sorption refrigeration system.

In principle, a sorption cycle is a four temperature cycle, but the two heat quantities $\mathrm{Q}_{\text {so }}$ and $\mathrm{Q}_{\mathrm{CO}}$, commonly rejected to the intermediate temperatures $\mathrm{T}_{\mathrm{SO}}$ and $\mathrm{T}_{\mathrm{CO}}$ respectively, are transferred to the same heat sink. In technical terms, the heat is removed from a cold water stream in a closed loop, passing serially from the generator, the condenser and the cooling tower. Thus, it is assumed that $\mathrm{T}_{\mathrm{SO}}=\mathrm{T}_{\mathrm{CO}}$ and the cycle is considered to be a three temperature cycle. The heat $\mathrm{Q}_{\mathrm{GE}}$ is supplied by the heat source (solar collectors) at temperature $\mathrm{T}_{\mathrm{GE}}$ to the thermal compressor (sorption system), where it induces desorption of the refrigerant vapour at the condenser pressure. The vapour is liquefied in the condenser at the intermediate temperature $\mathrm{T}_{\mathrm{CO}}$. When the sorption system is cooled down back to the intermediate temperature $\mathrm{T}_{\mathrm{SO}}$, it reabsorbs vapour, which is produced by the vaporisation of the liquefied refrigerant in the evaporator. The vaporisation heat $\mathrm{Q}_{\mathrm{EV}}$ derives from the cooling space, through the chilled water circulation. The coefficient of performance of a sorption refrigerator is expressed as the amount of cooling delivered (cold produced) divided by the amount of the required heat input:

$$
\mathrm{COP}=\frac{Q_{\mathrm{EV}}}{Q_{\mathrm{GE}}}
$$

where, $\mathrm{Q}_{\mathrm{EV}}$ denotes the heat removed from the cooled space; and $\mathrm{Q}_{\mathrm{GE}}$ denotes the heat derived from the heat source to the generator (desorber).

Based on the thermodynamic cycle of operation principle, absorption cycles are considered single-effect, half-effect, double-effect, and triple-effect [16,19]. Table 2 shows a comparison on the performance of different absorption cooling systems $[5,14,26]$. The single-effect system is the simplest type and the most used in commercial devices $[27,28]$. The half-effect cycle, also called two-stage or double-lift cycle, can provide cold with a relatively low driving temperature. The COP of this cycle is roughly half of the single-effect cycle and so often called half-effect. Single-effect absorption cooling system is based on the basic absorption cycle that contains a single absorber and generator as shown in Figure 5. In the generator G, the refrigerant is 
separated from the absorbent by the heat provided by the solar collector. The vapour-refrigerant are condensed in condenser $\mathrm{C}$, then laminated in expansion valve EV1 and evaporated at low pressure and temperature in the evaporator $\mathrm{V}$. The cooled refrigerant is absorbed in the absorber $\mathrm{Ab}$ by weak-solution that returns from generator after the lamination in the expansion valve EV2. The rich-mixture created in absorber is pumped by pump $\mathrm{P}$ and returned in $\mathrm{G}$. The usual a solution heat exchanger (SHX) can be used to improve cycle efficiency [16]. A 60\% higher COP can be achieved by using the SHX [29]. The absorption being exothermic, the absorber is chilled with cooling water. The half-effect absorption refrigeration cycle has discussed by Arivazhagan et al. [30] and Gebreslassie et al. [31].

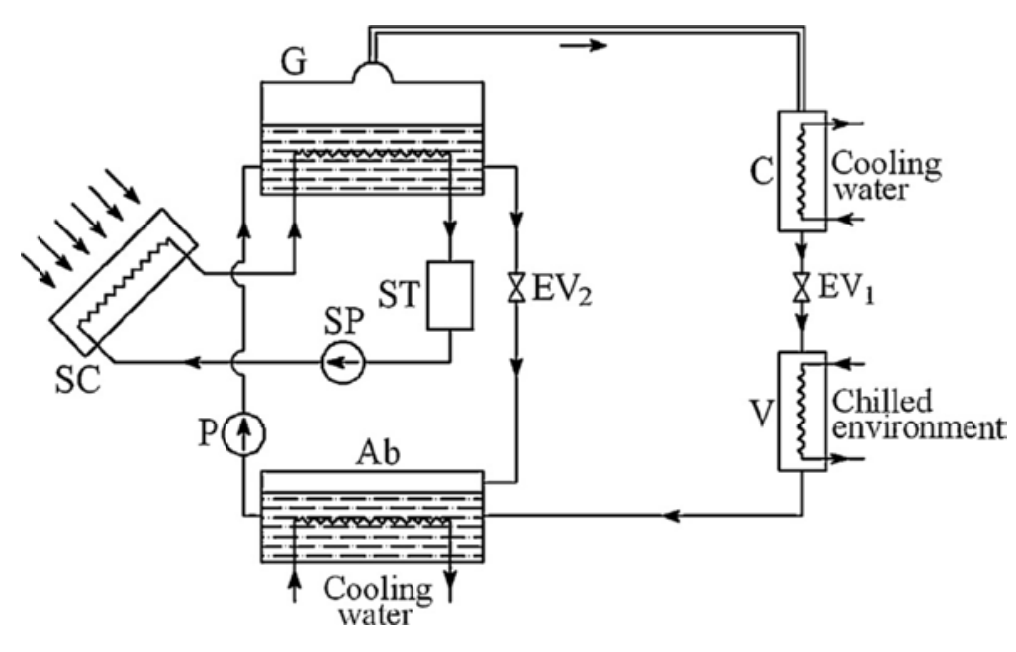

Figure 5. Schematic of solar absorption cooling systems [5,14].

Double-effect systems can be achieved by adding an extra stage as a topping cycle on the single-effect cycle. They have a double COP value compared with that of single-effect systems, nearly 1.2. However, their working liquids must achieve higher temperature, more than $130{ }^{\circ} \mathrm{C}$. Double-effect absorption cooling technology was launched in 1956 for developing the system performance within a heat source at higher temperature [32]. Figure 6 illustrates a double-effect absorption system with a $\mathrm{H}_{2} \mathrm{O} / \mathrm{LiBr}$ pair. The cycle begins with generator G-I providing heat to generator G-II. The condenser $\mathrm{C}$ rejects the heat and passes the working fluid towards the evaporator $\mathrm{V}$; within this step, the required refrigeration occurs. Then, the fluids pass through the heat exchangers HX-I and HX-II from the absorber Ab to G-I by means of a pump P. Through this process, HX-II can pass the fluids to G-II and then G-II passes to HX-I. The complete cycle follows three different pressure levels: high, medium and low. Many researchers have studied and analysed the double-effect absorption cooling machines [33,37]. 
225 Triple-effect absorption cooling can be classified as single-loop or dual-loop cycles [5,14].

226 Single-loop triple-effect cycles are basically double-effect cycles with an additional generator

227 and condenser. The resulting system with three generators and three condensers operates 228 similarly to the double-effect system. Primary heat concentrates absorbent solution in a first-

Figure 6. Schematic of solar assisted double-effect $\mathrm{H}_{2} \mathrm{O} / \mathrm{LiBr}$ absorption system [5,14]. stage generator at about $200-23{ }^{\circ} \mathrm{C}$. A fluid pair other than $\mathrm{H}_{2} \mathrm{O} / \mathrm{LiBr}$ must be used for the high temperature cycle. The refrigerant vapour produced is then used to concentrate additional absorbent solution in a second-stage generator at about $150{ }^{\circ} \mathrm{C}$. Finally, the refrigerant vapour produced in the second-stage generator concentrates additional absorbent solution in a thirdstage generator at about $93{ }^{\circ} \mathrm{C}$. The usual solution heat exchangers can be used to improve cycle efficiency. Theoretically, these triple-effect cycles can obtain COPs of about 1.7 [38].

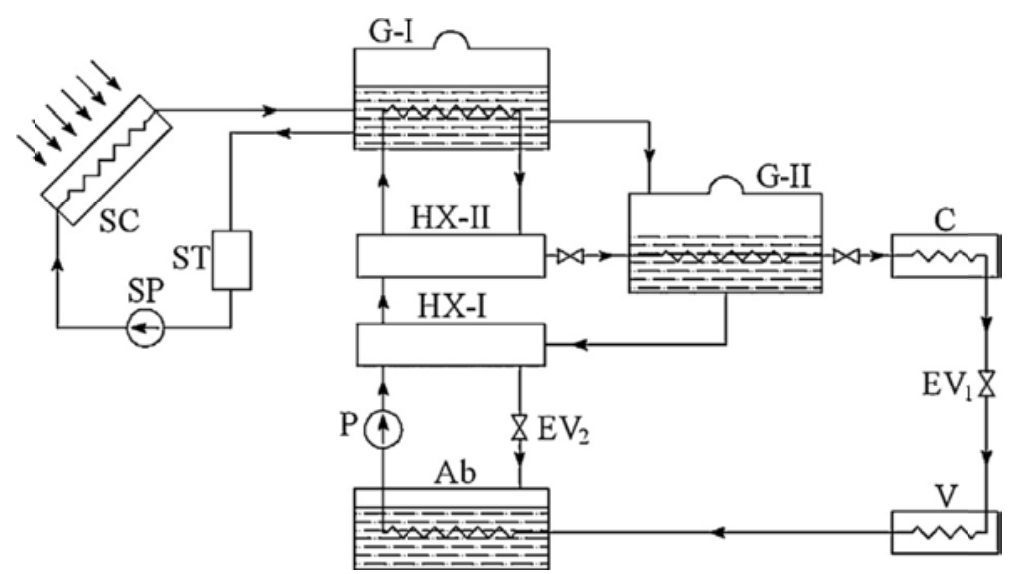


Table 2. Characteristics of absorption cycles.

\begin{tabular}{|c|c|c|c|c|c|c|c|c|c|c|c|}
\hline $\begin{array}{l}\text { Absorption } \\
\text { cooling }\end{array}$ & $\begin{array}{l}\mathrm{T}_{\text {evap }} \\
\left({ }^{\circ} \mathrm{C}\right)\end{array}$ & $\mathrm{T}_{\text {ref }}\left({ }^{\circ} \mathrm{C}\right)$ & $\begin{array}{l}\mathrm{T}_{\text {gen }} \\
\left({ }^{\circ} \mathrm{C}\right)\end{array}$ & $\begin{array}{l}\mathrm{T}_{\text {heating }} \\
\left({ }^{\circ} \mathrm{C}\right)\end{array}$ & $\begin{array}{c}X_{\text {weak }} \\
(\mathrm{kg} / \mathrm{kg})\end{array}$ & $\begin{array}{c}\mathrm{X}_{\text {strong }} \\
(\mathrm{kg} / \mathrm{kg})\end{array}$ & $\mathrm{COP}_{\text {Carnot }}$ & $\alpha$ & $\mathrm{COP}$ & $\begin{array}{c}\text { EER } \\
(\mathrm{Bry} / \mathrm{Wh})\end{array}$ & General remarks of the technology \\
\hline \multirow[t]{3}{*}{$\begin{array}{l}\mathrm{Half-effect} \\
\mathrm{H}_{2} \mathrm{O}-\mathrm{LiBr}\end{array}$} & \multirow{3}{*}{$2[26]$} & $30[26]$ & $57[26]$ & $67[26]$ & $\begin{array}{c}0.560 \\
{[26]}\end{array}$ & $0.472[26]$ & $0.80[26]$ & $0.44[26]$ & $0.35[26]$ & --- & $\begin{array}{c}\text { Within the optimum temperature range of } 65- \\
70^{\circ} \mathrm{C} \text {, the COP is } 0.36 \text { and the evaporation } \\
\text { temperature is }-7^{\circ} \mathrm{C}[5,30]\end{array}$ \\
\hline & & $40[26]$ & $78[26]$ & $88[26]$ & $\begin{array}{c}0.515 \\
{[26]}\end{array}$ & $0.418[26]$ & $0.78[26]$ & $0.44[26]$ & $0.34[26]$ & --- & $\begin{array}{l}\text { The pair is able to provide the same COP as a } \\
\text { conventional cooling system }[5,39]\end{array}$ \\
\hline & & $50[26]$ & $98[26]$ & $108[26]$ & $\begin{array}{c}0.470 \\
{[26]}\end{array}$ & $0.364[26]$ & $0.74[26]$ & $0.44[26]$ & $0.33[26]$ & --- & $\begin{array}{l}\text { The system has } 22 \% \text { lower exergetic efficiency } \\
\text { compared to the single-effect system }[5,40]\end{array}$ \\
\hline \multirow[t]{6}{*}{$\begin{array}{l}\text { Single-effect } \\
\mathrm{H}_{2} \mathrm{O}-\mathrm{LiBr}\end{array}$} & \multirow{6}{*}{$2[26]$} & $30[26]$ & $82[26]$ & $92[26]$ & $\begin{array}{c}0.440 \\
{[26]}\end{array}$ & $0.328[26]$ & $1.44[26]$ & $0.50[26]$ & $0.72[26]$ & --- & --- \\
\hline & & --- & --- & $\begin{array}{c}86.5 \\
{[5,14,42]}\end{array}$ & --- & --- & --- & --- & $\begin{array}{c}0.7 \\
{[5,14,42]}\end{array}$ & $\begin{array}{r}2.39 \\
{[5,14} \\
26,42]\end{array}$ & $\begin{array}{c}\text { A COP approximately } 60 \% \text { higher can be } \\
\text { achieved by using a vacuum tubular collector } \\
{[5,43]}\end{array}$ \\
\hline & & $40[26]$ & $94[26]$ & $104[26]$ & $\begin{array}{c}0.360 \\
{[26]}\end{array}$ & $0.328[26]$ & $1.06[26]$ & $0.50[26]$ & $0.53[26]$ & --- & $\begin{array}{l}\text { A system capacity of } 70 \mathrm{~kW} \text { can be achieved } \\
\text { by using a vacuum tubular collector }\left(108 \mathrm{~m}^{2}\right) \\
\text { with flat plate collectors } 9124 \mathrm{~m}^{2}[5,28]\end{array}$ \\
\hline & & $50[26]$ & --- & --- & --- & --- & --- & --- & --- & --- & $\begin{array}{l}\text { COP is increased by } 15 \% \text { using a portioned hot } \\
\text { water tank with a flat plate collector }[5,27]\end{array}$ \\
\hline & & --- & --- & $\begin{array}{c}80-100 \\
{[19]}\end{array}$ & --- & --- & --- & $\begin{array}{c}0.5-0.7 \\
{[19]}\end{array}$ & --- & --- & Simplest and widely used [19] \\
\hline & & --- & --- & --- & --- & --- & --- & --- & --- & --- & $\begin{array}{l}\text { Water cooled absorber is required to prevent } \\
\text { crystallization at higher concentration [19] }\end{array}$ \\
\hline \multirow[t]{4}{*}{$\begin{array}{l}\text { Single-effect } \\
\mathrm{NH}_{3}-\mathrm{H}_{2} \mathrm{O}\end{array}$} & \multirow{4}{*}{$2[26]$} & $30[26]$ & $76[26]$ & $86[26]$ & $\begin{array}{c}0.550 \\
{[26]}\end{array}$ & $0.460[26]$ & $1.29[26]$ & $0.46[26]$ & $0.60[26]$ & $-\overline{---}$ & Rectification of refrigerant is required [19] \\
\hline & & $40[26]$ & $108[26]$ & $118[26]$ & $\begin{array}{c}0.475 \\
{[26]}\end{array}$ & $0.370[26]$ & $1.29[26]$ & $0.46[26]$ & $0.59[26]$ & --- & $\begin{array}{c}\text { Working solution is environmental friendly } \\
{[19]}\end{array}$ \\
\hline & & $50[26]$ & $130[26]$ & $140[26]$ & $\begin{array}{c}0.425 \\
{[26]}\end{array}$ & $0.310[26]$ & $1.14[26]$ & $0.46[26]$ & $0.52[26]$ & --- & No crystallization problem [19] \\
\hline & & --- & --- & $\begin{array}{c}120-150 \\
{[19]}\end{array}$ & --- & --- & --- & --- & $0.5[19]$ & --- & $\begin{array}{l}\text { Suitable for using as heat pump due to with } \\
\text { operation range [19] }\end{array}$ \\
\hline \multirow[t]{3}{*}{$\begin{array}{l}\text { Double-effect } \\
\mathrm{H}_{2} \mathrm{O}-\mathrm{LiBr}\end{array}$} & \multirow{3}{*}{2} & $30[26]$ & $132[26]$ & 142 [26] & $\begin{array}{c}0.440 \\
{[26]}\end{array}$ & $0.328[26]$ & $2.47[26]$ & $0.52[26]$ & 1.29 [26] & --- & $\begin{array}{l}\text { The double-effect chillers with through } \\
\text { collectors show the maximum potential } \\
\text { savings }[5,44]\end{array}$ \\
\hline & & --- & --- & $\begin{array}{c}130 \\
{[5,14,42]}\end{array}$ & --- & --- & --- & --- & $\begin{array}{c}1.2 \\
{[5,14,42]}\end{array}$ & $\begin{array}{c}4.10 \\
{[5,14,42]}\end{array}$ & $\begin{array}{l}\text { The system has almost double }(0.96) \text { the COP } \\
\text { compared to the single-effect system }[5,19]\end{array}$ \\
\hline & & $40[26]$ & $166[26]$ & $176[26]$ & $\begin{array}{c}0.385 \\
{[26]}\end{array}$ & $0.300[26]$ & $2.08[26]$ & $0.52[26]$ & $1.08[26]$ & --- & $\begin{array}{l}\text { High performance cycle available } \\
\text { commercially [19] }\end{array}$ \\
\hline
\end{tabular}




\begin{tabular}{|c|c|c|c|c|c|c|c|c|c|c|c|}
\hline & & $50[26]$ & $\begin{array}{ll}-- \\
-1\end{array}$ & --- & +-- & --- & --- & --- & --- & --- & $\begin{array}{l}\text { Heat of condensation from the first effect is } \\
\text { used as heat input for the second stage [19] }\end{array}$ \\
\hline & & --- & --- & $\begin{array}{c}120-150 \\
{[19]}\end{array}$ & --- & --- & --- & --- & $\begin{array}{c}0.8-1.2 \\
{[19]}\end{array}$ & -- & --- \\
\hline \multirow[t]{3}{*}{ Triple-effect } & \multirow{3}{*}{---} & --- & --- & $\begin{array}{c}220 \\
{[5,14,42]}\end{array}$ & --- & --- & --- & --- & $\begin{array}{c}1.7 \\
{[5,14,42]}\end{array}$ & $\begin{array}{c}5.80 \\
{[5,14,42]}\end{array}$ & High complexity of the control system [19] \\
\hline & & --- & $\begin{array}{ll}-- \\
-1\end{array}$ & $\begin{array}{c}200-230 \\
{[19]}\end{array}$ & +-- & --- & --- & --- & $\begin{array}{c}1.4-1.5 \\
{[19]}\end{array}$ & --- & $\begin{array}{l}\text { Likely to be direct fired as the input } \\
\text { temperature is quite high [19] }\end{array}$ \\
\hline & & --- & --- & --- & --- & --- & --- & --- & --- & --- & $\begin{array}{l}\text { Requires more maintenance as a result of high } \\
\text { corrosion due to the operating temperature }[19\end{array}$ \\
\hline Hybrid & --- & --- & --- & --- & --- & --- & --- & --- & --- & --- & $\begin{array}{l}\text { The types of systems are widely implemented } \\
\text { for the cooling in larger places }[5,45]\end{array}$ \\
\hline
\end{tabular}


Infante Ferreira and Kim [26] carried out a techno-economic comparison of the different solar cooling technologies, including absorption and adsorption systems. Table 3 gives an estimation of the investment costs, including installation costs, of the several cooling cycle technologies. For comparison purposes, it also shows values proposed in recent publications. Some systems require an engine (Stirling or Rankine). A value of $1700 € / \mathrm{kW}$ shaft power was considered.

Table 3. Specific prices of different cooling cycles (thousands $€ / \mathbf{k W}$ ).

\begin{tabular}{|c|c|c|c|c|c|c|}
\hline $\begin{array}{c}\text { Vapour } \\
\text { compression }\end{array}$ & $\begin{array}{c}\text { Half-effect } \\
\text { absorption }\end{array}$ & $\begin{array}{c}\text { Single- } \\
\text { effect } \\
\text { absorption }\end{array}$ & $\begin{array}{c}\text { Double- } \\
\text { effect } \\
\text { absorption }\end{array}$ & Adsorption & Desiccant & Reference \\
\hline 300 & --- & 400 & --- & 850 & --- & {$[26,46]$} \\
\hline 200 & --- & 400 & 300 & 500 & --- & {$[25,26]$} \\
\hline 210 & --- & 250 & 550 & --- & --- & {$[26,47]$} \\
\hline--- & --- & --- & --- & --- & 370 & {$[26,48]$} \\
\hline--- & --- & 210 & 855 & 855 & 1065 & {$[26,49]$} \\
\hline 300 & --- & 400 & 700 & 600 & --- & {$[26,50]$} \\
\hline 250 & 700 & 400 & 700 & 700 & 700 & {$[26]$} \\
\hline
\end{tabular}

As example, the main features of the possible solar refrigeration technologies, with respect to their COP, application and input temperature needed are presented in Table 4 [4].

Table 4. Main features of solar sorption refrigeration technologies [4].

\begin{tabular}{|c|c|c|c|c|c|c|c|}
\hline & \multicolumn{4}{|c|}{ Absorption } & \multicolumn{3}{|c|}{ Adsorption } \\
\hline & \multicolumn{2}{|l|}{ LiBr/water } & \multicolumn{2}{|l|}{ Water/ $\mathbf{N H}_{3}$} & \multirow{2}{*}{$\begin{array}{l}\text { One-stage } \\
\text { (silica } \\
\text { gel/water) }\end{array}$} & \multirow{2}{*}{$\begin{array}{l}\text { Two-stage } \\
\text { (silica } \\
\text { gel/water) }\end{array}$} & \multirow{2}{*}{$\begin{array}{l}\text { Adsorber } \\
\text { /collector }\end{array}$} \\
\hline & $\begin{array}{l}\text { Single- } \\
\text { effect }\end{array}$ & $\begin{array}{l}\text { Double- } \\
\text { effect }\end{array}$ & $\begin{array}{l}\text { Single- } \\
\text { stage }\end{array}$ & Two-stage & & & \\
\hline COP & $0.5-0.75$ & $0.8-1.2$ & 0.5 & $1.2-1.3$ & $0.3-0.7$ & 0.35 & $0.1-0.5$ \\
\hline $\begin{array}{l}\text { Applicatio } \\
\mathrm{n}\end{array}$ & $\begin{array}{l}\text { Air- } \\
\text { conditionin } \\
\mathrm{g}\end{array}$ & $\begin{array}{l}\text { Air- } \\
\text { conditionin } \\
\mathrm{g}\end{array}$ & $\begin{array}{l}\text { Air- } \\
\text { conditioning } \\
\text { Refrigeratio } \\
\mathrm{n}\end{array}$ & $\begin{array}{l}\text { Air- } \\
\text { conditioning } \\
\text { Refrigeratio } \\
\mathrm{n}\end{array}$ & $\begin{array}{l}\text { Air- } \\
\text { conditionin } \\
\mathrm{g}\end{array}$ & $\begin{array}{l}\text { Air- } \\
\text { conditionin } \\
\mathrm{g}\end{array}$ & $\begin{array}{l}\text { Refrigeratio } \\
\mathrm{n}\end{array}$ \\
\hline $\begin{array}{l}\text { Input } \\
\text { temperatur } \\
\text { e }\left({ }^{\circ} \mathrm{C}\right)\end{array}$ & $80-100$ & $100-160$ & $120-150$ & & $60-90$ & $50-75$ & \\
\hline
\end{tabular}




\subsection{Materials}

\subsubsection{Absorption systems}

There are two main absorption systems: lithium bromide/water and water-ammonia [14,14,16,52 -54]. A comparison between both systems is presented in Table 5. Broadly speaking, $\mathrm{NH}_{3}-\mathrm{H}_{2} \mathrm{O}$ systems are often used for refrigeration and in industrial applications while $\mathrm{H}_{2} \mathrm{O}-$ $\mathrm{LiBr}$ systems are more suitable for air-conditioning purposes.

Table 5.Comparison between the absorption systems with $\mathrm{NH}_{3}-\mathrm{H}_{2} \mathrm{O}$ and $\mathrm{H}_{2} \mathrm{O}-\mathrm{LiBr}[1,5]$

\begin{tabular}{|l|l|l|}
\hline & Water/NH & Li/water \\
\hline Advantages & $\begin{array}{l}\text { Evaporative at the temperature } \\
\text { below } 0^{\circ} \mathrm{C}\end{array}$ & $\begin{array}{l}\text { High COP } \\
\text { Low operation pressures } \\
\text { Environmental friendly and innoxious } \\
\text { Large latent heat of vaporization }\end{array}$ \\
\hline Disadvantages & $\begin{array}{l}\text { Toxic and dangerous for health } \\
\text { In need of a column of rectifier } \\
\text { Operation at high pressure }\end{array}$ & $\begin{array}{l}\text { The risk of congelation } \\
\text { Relatively expensive }(\mathrm{LiBr})\end{array}$ \\
\hline
\end{tabular}

The basic lithium bromide-water ( $\mathrm{LiBr}$ ) absorption cycle has been in use in refrigeration applications since the 1940s. Its key characteristics are determined by the pressure-temperature concentration (PTX) equilibrium chart, the solution cycles, the individual components and the chemical additives. Its functional performance is mainly characterised by the partial load operation and the particularities of maintenance of the system. In this cycle, $\mathrm{LiBr}$ is the absorbent and water is the refrigerant. The output of a $\mathrm{LiBr}$ system can be as low as $4{ }^{\circ} \mathrm{C}$. These absorption systems are categorised by the number of times the solution is heated to produce refrigerant vapours, referred to as the number of effects. Figure 7 shows a typical single-effect solar driven $\mathrm{LiBr}-$ water absorption refrigeration system.

The second widely used refrigeration system is based on the water-ammonia absorption cycle. This system is mainly encountered in industrial applications, in chemical processes, in the food industry and in drying processes. It is also suitable for solar energy applications. In the case of a water-ammonia absorption cycle, water is the absorbent and ammonia is the refrigerant (i.e. the opposite of the $\mathrm{LiBr}$ cycle). The cooling output in most cases is liquid ammonia, separated from the ammonia in the water-ammonia absorption cycle machine, as opposed to the chilled water used in $\mathrm{LiBr}$ absorption systems. Due to the fact that ammonia is the refrigerant, the output of a water-ammonia absorption cycle system can be as low as $-60^{\circ} \mathrm{C}$. If the system is installed in a closed space, ventilation is required by the standards for ammonia refrigeration. Waterammonia absorption cycle systems are categorised according to the number of evaporator 
stages. A single-stage system has one stage of evaporation/absorption, and a two-stage system has two stages of evaporation/absorption. The number of stages of evaporation can be increased in order to reach the cooling and the refrigeration temperature required by the design parameters. Water-ammonia absorption cycle systems are tailor-made systems for each application. The operational principles of a water-ammonia absorption cycle system are similar to those of $\mathrm{LiBr}$ absorption machines.

On the other hand, Du et al. [51] proposed an air-cooled two-stage $\mathrm{NH}_{3}-\mathrm{H}_{2} \mathrm{O}$ absorption refrigeration system is proposed for potential application of residential small scale cooling system driven by solar heated hot water. The study reveals the technical feasibility of the aircooled two-stage NH3-H2O absorption system and provides an alternative low-cost small bulk solar absorption air-conditioning.

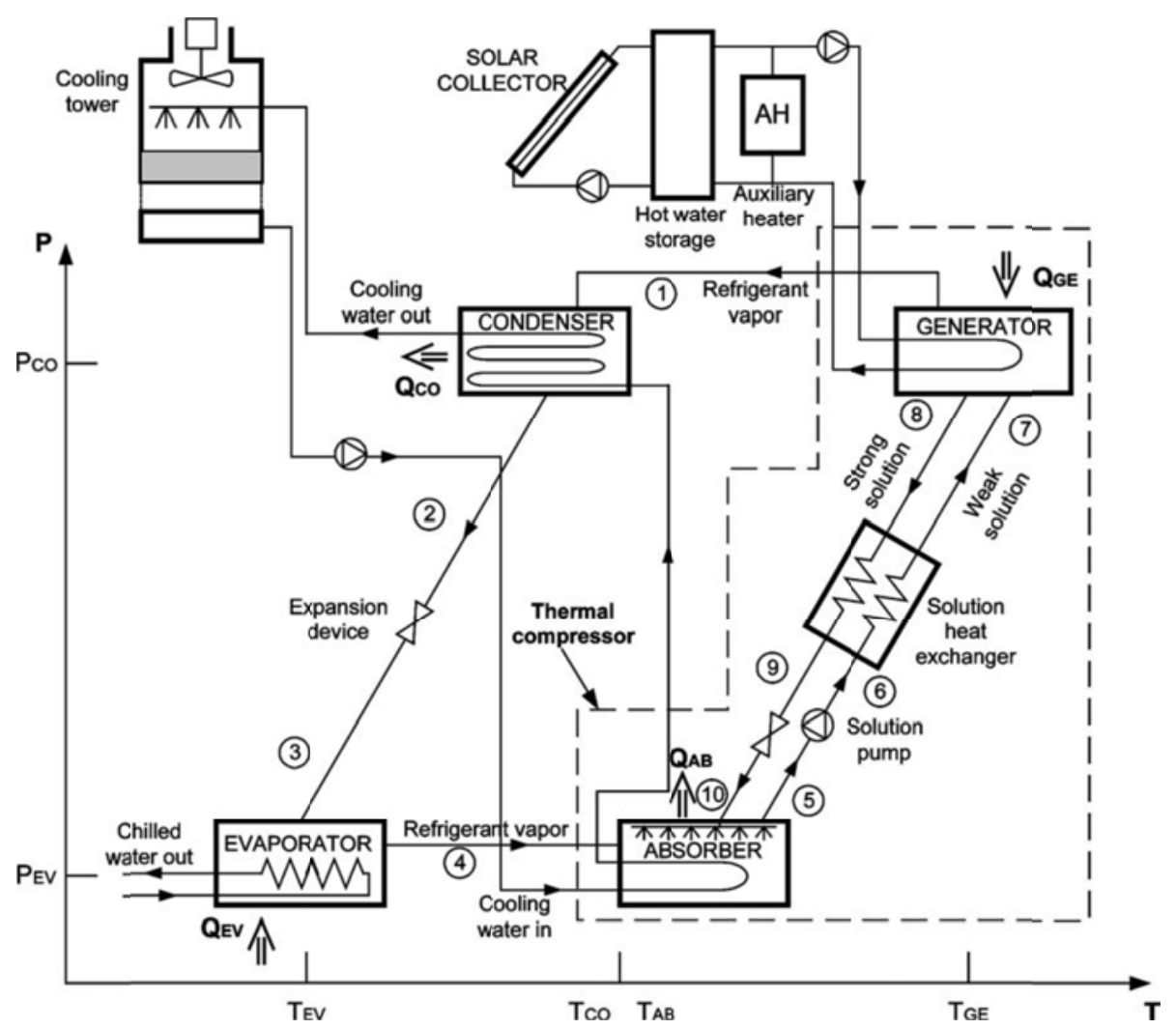

Figure 7. Schematic of a solar driven LiBr-water single-effect absorption refrigeration System [4]. 
Table 6. Overview of solar absorption refrigeration studies with $\mathrm{LiBr} / \mathrm{H}_{2} \mathrm{O}$ and $\mathrm{NH}_{3} / \mathrm{H}_{2} \mathrm{O}$

\begin{tabular}{|c|c|c|c|c|c|c|}
\hline Technology & Application & $Q_{e}[k W]$ & $\mathbf{A}_{\mathrm{s}}\left[\mathrm{m}^{2}\right]$ & $\begin{array}{l}\text { Efficiency - } \\
\eta_{\text {heat-cool }}\end{array}$ & COP & Reference \\
\hline \multirow{11}{*}{$\begin{array}{l}\text { Single-effect } \\
\mathrm{LiBr} / \mathrm{H} 2 \mathrm{O}\end{array}$} & Space cooling/ heating & --- & --- & --- & --- & {$[16,25,58-64]$} \\
\hline & \multirow{8}{*}{ Space cooling } & 4 & 36 & 0.11 & --- & {$[25,65]$} \\
\hline & & 210 & 1577 & 0.31 & --- & {$[25,66]$} \\
\hline & & 90 & 316 & $0.26-0.36$ & --- & {$[25,67]$} \\
\hline & & 35 & 49.9 & 0.34 & --- & {$[25,68]$} \\
\hline & & --- & --- & --- & 0.490 & {$[5,69]$} \\
\hline & & --- & --- & --- & 0.620 & {$[5,70]$} \\
\hline & & --- & --- & --- & 0.660 & {$[5,71]$} \\
\hline & & --- & --- & --- & 0.740 & {$[5,72]$} \\
\hline & \multirow[t]{2}{*}{ Prototype chiller } & 10 & --- & 0.37 & --- & {$[25,73,74]$} \\
\hline & & 16 & --- & 0.40 & --- & {$[25,75]$} \\
\hline \multirow[t]{2}{*}{$\begin{array}{l}\text { Double-effect } \\
\mathrm{LiBr} / \mathrm{H} 2 \mathrm{O}\end{array}$} & $\begin{array}{l}\text { Fuel-fired-solar-assisted } \\
\text { prototype }\end{array}$ & --- & --- & --- & --- & {$[25,76,77]$} \\
\hline & $\begin{array}{l}\text { Cooling/steam } \\
\text { generation }\end{array}$ & 140 & 180 & $0.5-0.6$ & --- & {$[25,78]$} \\
\hline \multirow[t]{6}{*}{$\mathrm{NH} 3 / \mathrm{H} 2 \mathrm{O}$} & $\begin{array}{l}\text { Diffusion/absorption } \\
\text { prototype }\end{array}$ & $<2.5$ & --- & $0.1-0.25$ & --- & {$[25,79-81]$} \\
\hline & Refrigeration/ heat pump & --- & --- & --- & --- & {$[16,25,79,82-88]$} \\
\hline & Wine cooling & 10 & 100 & $\begin{array}{ll}-- \\
\end{array}$ & --- & {$[5,89]$} \\
\hline & \multirow[t]{3}{*}{ Space cooling } & 15 & --- & 0.27 & --- & {$[5,90]$} \\
\hline & & --- & --- & --- & 0.427 & {$[5,91]$} \\
\hline & & --- & --- & --- & 0.550 & {$[5,92]$} \\
\hline
\end{tabular}

Table 7. Other working pairs for absorption

\begin{tabular}{|c|c|c|c|}
\hline Working pair & COP & Comments & Reference \\
\hline $\mathrm{NH} 3 / \mathrm{CaCl}_{2}$ & 0.10 & $\begin{array}{l}\text { Ice production } 6 \mathrm{~kg} / \mathrm{m} 2 \\
\text { of collector area }\end{array}$ & {$[1,16,93]$} \\
\hline \multirow[t]{2}{*}{$\mathrm{NH} 3 / \mathrm{SrCl}_{2}$} & --- & --- & {$[1,93]$} \\
\hline & $\begin{array}{l}0.049(1994) \\
0.045-0.082(1995)\end{array}$ & --- & {$[1,16,94]$} \\
\hline NH3/IMPEX $\left(80 \% \mathrm{SrCl}_{2}+20 \%\right.$ graphite & 0.143 & $1.45 \mathrm{kWh} /$ day & {$[1,16,95]$} \\
\hline $\begin{array}{l}\text { Organic fluid mixtures } \\
\text { trifluoroethanol (TFE)-tetraethylenglycol } \\
\text { dimethylether (TEGDME or E181) and } \\
\text { methanol-TEGDME }\end{array}$ & $\begin{array}{l}15 \% \text { higher than NH3- } \\
\mathrm{H}_{2} \mathrm{O}\end{array}$ & Simulations & {$[1,16,96,97]$} \\
\hline $\begin{array}{l}\text { Aqueous ternary hydroxide 40:36:24 } \\
(\mathrm{NaOH}: \mathrm{KOH}: \mathrm{CsOH})\end{array}$ & Higher than $\mathrm{NH} 3-\mathrm{H} 2 \mathrm{O}$ & $\begin{array}{l}\text { Operates at broader } \\
\text { temperatures than NH3- } \\
\mathrm{H}_{2} \mathrm{O}\end{array}$ & {$[1,16,98]$} \\
\hline NH3/LiNO3 mixture & $0.15-0.4$ & Produce $11.8 \mathrm{~kg}$ of ice & {$[1,16,99]$} \\
\hline NH3/Water-NaOH mixture & --- & --- & {$[16,100]$} \\
\hline $\begin{array}{l}\text { H2O/Ionic liquid } \quad \begin{array}{l}\text { (1-ethyl-3- } \\
\text { methilimidazolium } \\
{[\text { EMIM][DMP] }}\end{array} \\
\end{array}$ & --- & --- & {$[16,101,102]$} \\
\hline $\begin{array}{l}\mathrm{H} 2 \mathrm{O} / \text { Ionic liquid } \\
\text { methylimidazolium ethylsulfate [EMISE]) }\end{array}$ & --- & --- & {$[16,103]$} \\
\hline H2O/Ethyleneglycol & --- & --- & {$[16,104]$} \\
\hline $\mathrm{H} 2 \mathrm{O} /$ Monomethylamine & --- & --- & {$[16,105]$} \\
\hline
\end{tabular}




\subsubsection{Adsorption systems}

In principle there are two broad categories of adsorption systems: continuous and intermittent, with intermittent systems being more suitable for the utilisation of solar energy since they are daily cycle systems $[2,4,56]$. Another structural categorisation of adsorption systems concerns the nature of the adsorption effect and particularly whether or not it is a physical phenomenon or whether or not it includes a chemical reaction [4,22,106-111]. When fixed adsorbent beds are employed, which is the common practice, these cycles can be operational without moving parts other than magnetic valves. This results in low vibration, mechanical simplicity, high reliability and a very long lifetime. The use of fixed beds also results in intermittent cycle operation, with adsorbent beds changing between adsorption and desorption stages. Hence, when constant flow of vapour from the evaporator is required in order to provide continuous cooling, two or more adsorbent beds must be operated out of phase [112]. Compared to absorption, adsorption chillers are more expensive and their commercial availability is still limited. Their COP is lower than that of the absorption chillers, but they can utilise heat at lower temperature, they can thus be driven by flat plate solar collectors more efficiently. Figure 8 shows a single-stage silica gel/water adsorption chiller driven by solar energy.

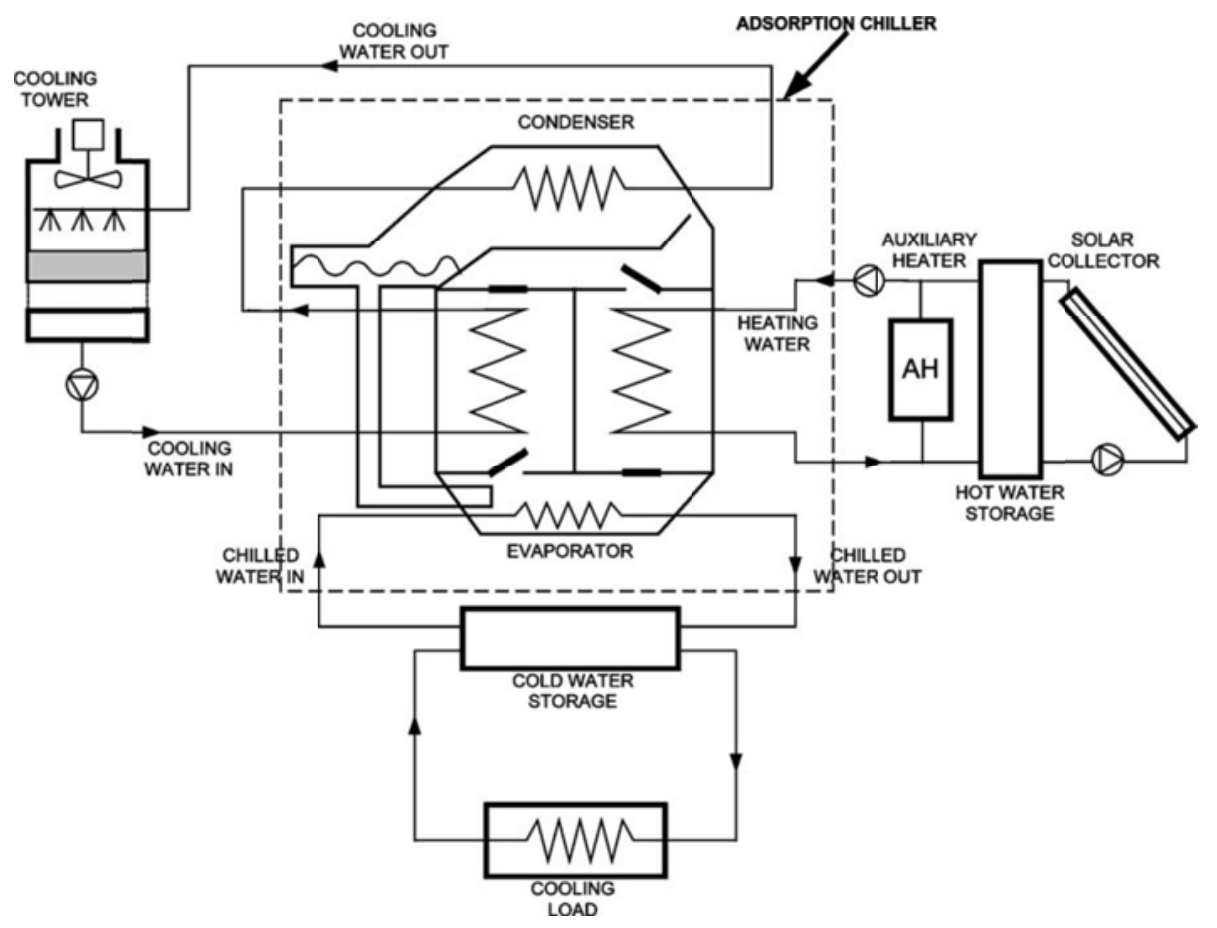

Figure 8. Schematic of a single-stage silica gel/water adsorption chiller [4].

In addition, Pan et al. [113] developed a silica gel-water adsorption chiller driven by low-grade heat. This chiller was tested under different conditions and it features the periodic variations of 
temperatures and cooling power and the obtained cooling power, COP and SCP are $42.8 \mathrm{~kW}$, 0.51 and $125.0 \mathrm{~W} / \mathrm{kg}$, respectively which were in range with those described in the literature.

Physical adsorption is caused by van de Walls force between the molecules of the adsorbent and the adsorbate [22,114]. Physical adsorbents with mesopores can adsorb consecutives layers of adsorbate, while those with micropores have the volume of the pores filled with the adsorbate. Physical adsorbents develop the selectivity to the adsorbate after the former undergo specific treatments, like react under a gas stream or with certain agents. The kind of treatment will depend on the type of sorbents [115]. Chemical adsorption is caused by the reaction between adsorbates and the surface molecules of adsorbents. Electron transfer, atom rearrangement and fracture or formation of chemical bond always occurs in the process of chemical adsorption [116]. Only one layer of adsorbate reacts with the surface molecules of chemical adsorbent. The adsorbate and adsorbent molecules after adsorption never keep their original state, e.g., complexation occurs between chlorides and ammonia. Moreover, there are the phenomena of salt swelling and agglomeration, which are critical to heat and mass transfer performance.

Composite adsorbents [117-119] started to be studied about in the late $80 \mathrm{~s}$ [120], and they aimed to improve the heat and mass transfer performance of the original chemical adsorbents $[118,121,122]$. This kind of adsorbent is usually obtained by the combination of a chemical adsorbent and a porous medium, that can be or not a physical adsorbent, such as activated carbon, graphite, carbon fibre, etc. [118,123,124].

Physical adsorbents used are activated carbon, activated carbon fibre, silica gel, and zeolite [22]. The activated carbon is made of materials such as wood, peat, coal, fossil oil, chark, bone, coconut shell and nut stone. The structure of activated carbon is shown in Figure 9a. The microcrystal for the activated carbon produced from bone is a six element carboatomic ring [115], and the adsorption performance is influenced by the functional groups that are connected to the carboatomic ring. The silica gel is a type of amorphous synthetic silica. It is a rigid, continuous net of colloidal silica (Figure 9b), connected to very small grains of hydrated $\mathrm{SiO}_{4}$ [125]. The hydroxyl in the structure is the adsorption centre because it is polar and can form hydrogen bonds with polar oxides, such as water and alcohol. The adsorption ability of silica gel increases when the polarity increases. One hydroxyl can adsorb one molecule of water. Zeolite is a type of aluminasilicate crystal composed of alkali or alkali soil. The chemical formula of zeolite is:

$$
\mathrm{M}_{y / n}\left[\left(\mathrm{AlO}_{2}\right)_{y}\left(\mathrm{SiO}_{2}\right)_{m}\right] z \mathrm{H}_{2} \mathrm{O}
$$

Eq. 6 
where $\mathrm{y}$ and $\mathrm{m}$ are all integers and $\mathrm{m} / \mathrm{y}$ is equal or larger than $1 ; \mathrm{n}$ is the chemical valence of positive ion of $\mathrm{M}$; and $\mathrm{z}$ is the number of water molecule inside a crystal cell unit. A crystal cell unit of zeolite is shown in Figure 9c. The positive ion must have its electric charge balanced with the electric charge of aluminium atom. The net electric charge of each aluminium atom is 1. Water can be removed by heating. There are about 40 types of natural zeollites, and the main types for adsorption refrigeration are chabazite, sodium chabazite, cowlesite and faujasite. About 150 types of zeolitescan be artificially synthesized, and they are named by one letter or a group of letters, such as type A, type X, type Y, type ZSM, etc. [115].

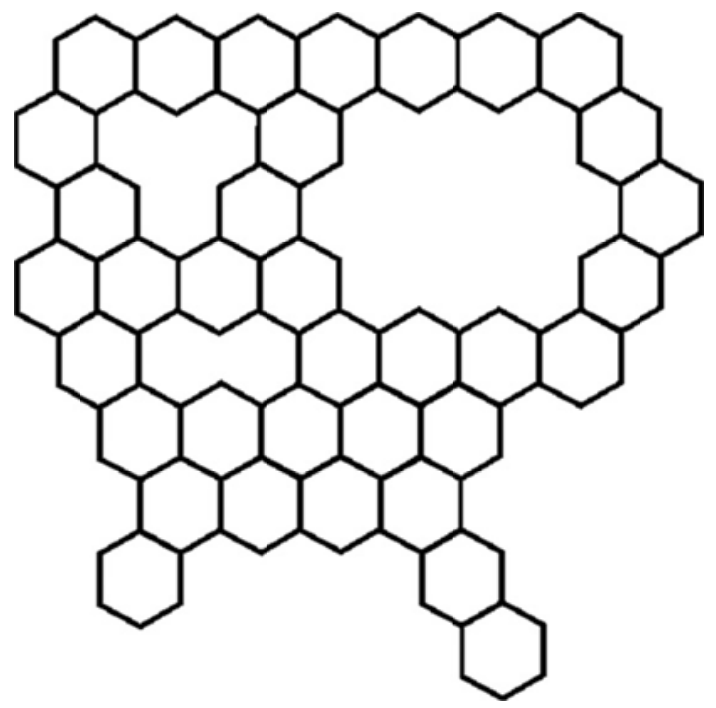

(a)



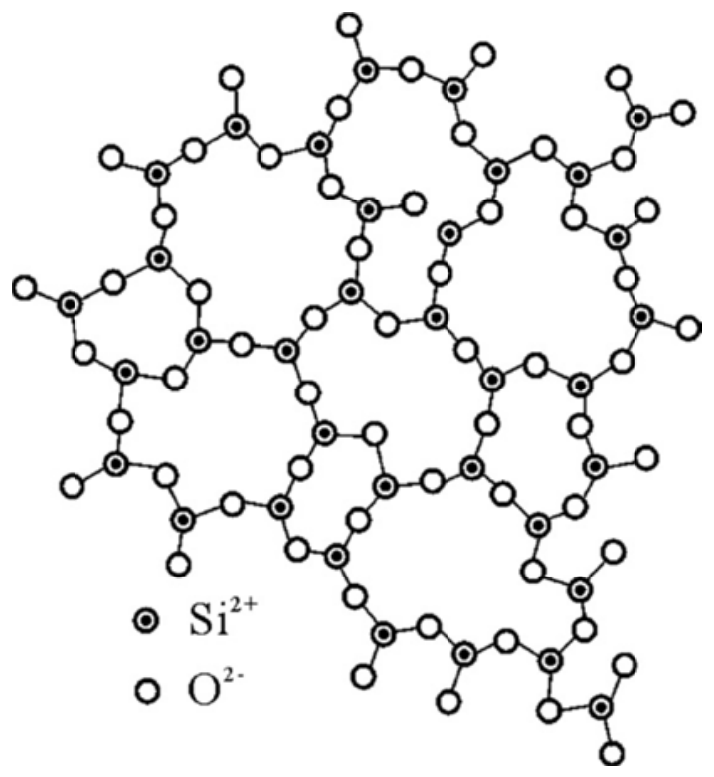

(b)

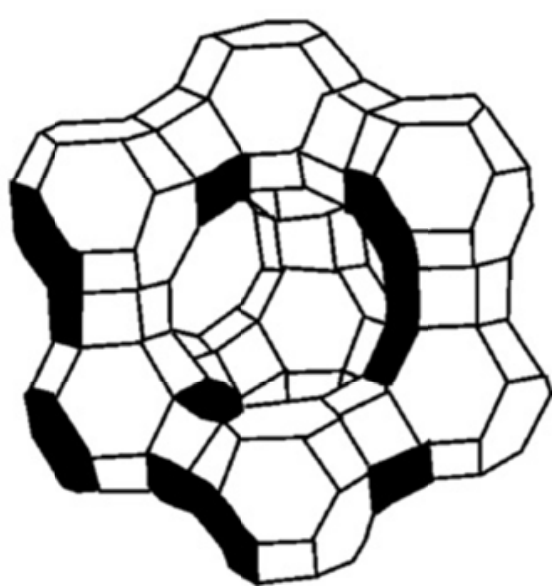

(c)

Figure 9. (a) Structure of activated carbon; (b) Array of SiO4 in silica gel; (c) Crystal cell unit of zeolite: left: crystal cell unit of type A zeolite; right: crystal cell unit of type $X$, Y seolity or faujasite $[22,125]$. 
Chemical adsorbents mainly include metal chlorides, metal hydrides, and metal oxides [22]. The metal chlorides for adsorption refrigeration are mainly calcium chloride, strontium chloride, magnesium chloride and barium chloride [126]. The adsorption reaction between metal chlorides and refrigerants is a complexation reaction, and the complex compound is also called coordinated compound [127]. Salt hydrides and metal hydrides can be utilized for adsorption refrigeration; in the adsorption process, $\mathrm{H}$ atoms become protons when they enter the space between hexagonal crystal lattices. When the metal oxides are used as adsorbents in adsorption heat pump $[111,128,129]$, oxygen is the refrigerant. On the surface of metal oxides, the elements which influence the adsorption performance are the coordination number of the metal ion, the unsaturated degree of coordination, the direction of the chemical bond on the surface of the chemical material, the symmetrical characteristic of the transition metal ligand field, the number of $\mathrm{d}$ electrons of the transition metal ligand field, and the arrangement of the active centres [130].

Composite adsorbents are developed and studied with mainly two goals [22]: to improve heat and mass transfer performance of chemical adsorbents [131], especially due to the swelling and agglomeration phenomena, and to increase the adsorption quantity of physical adsorbents [132]. The composite adsorbents made from porous media and chemical sorbents are commonly a combination of metal chlorides and activated carbon, or activated carbon fibre, or expanded graphite, or silica gel or zeolite.

On the other hand, the common refrigerants used in adsorption systems are ammonia, water, and methanol [22]. Hydrogen and oxygen are examples of other refrigerants that can be adopted in adsorption refrigeration systems. Other refrigerants, as R134a, R22, R407c and ethanol can be utilized if activated carbon or activated carbon fibre is the sorbent.

Table 8 presents a summary of conventional working pairs for solid-gas sorption $[1,5,9,19,22]$. 
Table 8. Summary of conventional working pairs for solid-gas sorption $[1,5,9,19,22]$

\begin{tabular}{|c|c|c|c|c|c|}
\hline & Working pair & $\begin{array}{l}\text { Heat source } \\
\text { temperature }\end{array}$ & Application & Advantages & Disadvantages \\
\hline \multirow[t]{5}{*}{$\begin{array}{l}\text { Physical } \\
\text { sorption } \\
\text { working pairs }\end{array}$} & $\begin{array}{l}\text { Silica } \\
\text { gel/water }\end{array}$ & $60-85^{\circ} \mathrm{C}$ & air-conditioning & $\begin{array}{l}\text { high latent heat of vaporization of } \\
\text { water, suitable for solar-powered } \\
\text { cooling systems and desiccant } \\
\text { cooling systems, the reutilization of } \\
\text { low-temperature waste heat }\end{array}$ & $\begin{array}{l}\text { unsuitable for the refrigeration application below } \\
0{ }^{\circ} \mathrm{C} \text {, vacuum system and it is very sensitive to } \\
\text { the leakage risk }\end{array}$ \\
\hline & Zeolite/water & $>150-200{ }^{\circ} \mathrm{C}$ & air-conditioning & $\begin{array}{l}\text { high latent heat of vaporization of } \\
\text { water, suitable for the reutilization } \\
\text { of high-temperature exhaust gas } \\
\text { and desiccant cooling systems }\end{array}$ & $\begin{array}{l}\text { only used for the refrigeration higher than } 0^{\circ} \mathrm{C} \text {, } \\
\text { high desorption temperature }\end{array}$ \\
\hline & $\begin{array}{l}\mathrm{AC} / \mathrm{AC} \\
\text { fibre/methan } \\
\text { ol }\end{array}$ & $80-110^{\circ} \mathrm{C}$ & $\begin{array}{l}\text { air-conditioning, } \\
\text { refrigeration, ice- } \\
\text { making }\end{array}$ & $\begin{array}{l}\text { large sorption capacity, low } \\
\text { adsorption heat, suitable for solar- } \\
\text { powered refrigeration systems }\end{array}$ & $\begin{array}{l}\text { low latent heat of vaporization of methanol, } \\
\text { unsuitable for heat source temperature higher } \\
\text { than } 120^{\circ} \mathrm{C}\end{array}$ \\
\hline & $\begin{array}{l}\mathrm{AC} / \mathrm{AC} \\
\text { fibre/ammoni } \\
\text { a }\end{array}$ & $>130-150{ }^{\circ} \mathrm{C}$ & $\begin{array}{l}\text { air-conditioning, } \\
\text { refrigeration, ice- } \\
\text { making }\end{array}$ & $\begin{array}{l}\text { good mass transfer, relative high } \\
\text { latent heat of vaporization of } \\
\text { ammonia, wide range of heat } \\
\text { source temperature }\end{array}$ & $\begin{array}{l}\text { toxicity and pungent smells of ammonia, low } \\
\text { sorption capacity, the incompatibility with copper } \\
\text { used in heat exchanger }\end{array}$ \\
\hline & $\begin{array}{l}\mathrm{AC} / \mathrm{AC} \\
\text { fibre/R134a }\end{array}$ & $>90-100^{\circ} \mathrm{C}$ & $\begin{array}{l}\text { air-conditioning, } \\
\text { refrigeration, ice- } \\
\text { making }\end{array}$ & $\begin{array}{l}\text { good mass transfer, wide range of } \\
\text { heat source temperature }\end{array}$ & $\begin{array}{l}\text { low sorption capacity and cooling power per } \\
\text { mass unit, low latent heat of vaporization of } \\
\text { refrigerant, relative high GWP value }\end{array}$ \\
\hline \multirow[t]{3}{*}{$\begin{array}{l}\text { Thermochemi } \\
\text { cal sorption } \\
\text { working pairs }\end{array}$} & $\begin{array}{l}\text { Metal } \\
\text { chloride/bro } \\
\text { mide/iodide- } \\
\text { ammonia* }\end{array}$ & $>50-350{ }^{\circ} \mathrm{C}^{+}$ & $\begin{array}{l}\text { air-conditioning, } \\
\text { refrigeration, ice- } \\
\text { making, deep- } \\
\text { freezing }\end{array}$ & $\begin{array}{l}\text { high sorption capacity per mass of } \\
\text { sorbent, wider range of heat source } \\
\text { temperature, suitable for } \\
\text { refrigeration and heat transformer. }\end{array}$ & $\begin{array}{l}\text { the swelling and agglomeration of salt, poor heat } \\
\text { and mass transfer, reduction in the sorption } \\
\text { capacity after many cycles }\end{array}$ \\
\hline & $\begin{array}{l}\text { Metal } \\
\text { oxides- } \\
\text { oxygen/water } \\
\text { /carbon } \\
\text { dioxide** }\end{array}$ & $\begin{array}{l}>150-1000 \\
{ }^{o} \mathrm{C}^{+}\end{array}$ & $\begin{array}{l}\text { refrigeration, } \\
\text { cryogenic, high- } \\
\text { temperature heat } \\
\text { pump }\end{array}$ & $\begin{array}{l}\text { high sorption capacity, wider range } \\
\text { of heat source temperature, suitable } \\
\text { for cryogenic conditions and high- } \\
\text { temperature heat pump }\end{array}$ & $\begin{array}{l}\text { the swelling and agglomeration of salt, poor heat } \\
\text { and mass transfer, reduction in sorption capacity } \\
\text { after many cycles, unsuitable for low-temperature } \\
\text { heat source due to their high desorption } \\
\text { temperature }\end{array}$ \\
\hline & Metal & $>110-400$ & air-conditioning, & fast sorption kinetics, high reaction & low specific cooling power per mass of sorbent, \\
\hline
\end{tabular}




\begin{tabular}{|c|c|c|c|c|c|}
\hline & $\begin{array}{l}\text { hydrides- } \\
\text { hydrogen*** }\end{array}$ & ${ }^{\circ} \mathrm{C}^{+}$ & $\begin{array}{l}\text { refrigeration, } \\
\text { deep-freezing, } \\
\text { heat pump }\end{array}$ & $\begin{array}{l}\text { heat for heat pump, high packed } \\
\text { density, wider range of heat source } \\
\text { temperature }\end{array}$ & $\begin{array}{l}\text { the swelling and agglomeration of salt, poor } \\
\text { thermal conductivity after many cycles }\end{array}$ \\
\hline \multirow[t]{2}{*}{$\begin{array}{l}\text { Composite } \\
\text { sorption } \\
\text { working pairs }\end{array}$} & $\begin{array}{l}\text { Silica } \\
\text { gel/metal } \\
\text { chlorides/wat } \\
\text { er**4 }\end{array}$ & $60-85^{\circ} \mathrm{C}$ & air-conditioning & $\begin{array}{l}\text { high sorption capacity per mass of } \\
\text { composite sorbent, high latent heat } \\
\text { of vaporization of water, suitable } \\
\text { for solar-powered cooling systems } \\
\text { and desiccant cooling systems }\end{array}$ & $\begin{array}{l}\text { only used for the refrigeration higher than } 0^{\circ} \mathrm{C} \text {, } \\
\text { poor heat transfer of composite sorbent, and the } \\
\text { risk of salt solution leakage from composite } \\
\text { sorbent }\end{array}$ \\
\hline & $\begin{array}{l}\text { Metal } \\
\text { chlorides/por } \\
\text { ous matrix- } \\
\text { ammonia*5 }\end{array}$ & $>50-350{ }^{\circ} \mathrm{C}^{+}$ & $\begin{array}{l}\text { air-conditioning, } \\
\text { refrigeration, ice- } \\
\text { making, deep- } \\
\text { freezing, heat } \\
\text { pump, heat } \\
\text { transformer }\end{array}$ & $\begin{array}{l}\text { high sorption capacity per mass of } \\
\text { composite sorbent, good heat and } \\
\text { mass transfer of composite sorbent, } \\
\text { wider range of heat source } \\
\text { temperature }\end{array}$ & $\begin{array}{l}\text { the complex fabrication of composite sorbent } \\
\text { using mixture or impregnation and consolidation, } \\
\text { the incompatibility with the conventional } \\
\text { material of copper used in heat exchanger }\end{array}$ \\
\hline
\end{tabular}

* The common metal chlorides include calcium chloride, barium chloride strontium chloride, magnesium chloride, nickel chloride, etc.

** The common metal oxides include calcium oxide, lead oxide, magnesium oxide, titanium oxide, etc.

*** The common metal hydrides include advanced porous metal hydrides (PMHs) and the misch metal (Mm) matrix alloys containing Ni, Fe, La, Al, $\mathrm{H}$, etc.

${ }^{4}$ The common metal chlorides include lithium chloride and calcium chloride, etc.

${ }^{5}$ The common porous matrix includes activated carbon, expanded graphite, activated carbon fibers, vermiculite, metallic foams, etc.

${ }^{+}$driving temperature depends on different working pairs 
Table 9 shows a comparison between adsorption working pairs; COP, SCP, delivered evaporation temperature, and required driving source temperature are presented. COP of the adsorption cooling systems achieved a maximum of 0.83 when employing metal hydrides/hydrogen pair, while the maximum SCP is achieved with AC/ammonia pair. It can be seen that most of the adsorption cooling systems are good to be driven by a low-grade heat source temperature lower than $100^{\circ} \mathrm{C}$. On the other hand, the lowest evaporator temperature is $50{ }^{\circ} \mathrm{C}$, produced with the metal hydrides/hydrogen pair (since the hydrogen has a very low normal boiling temperature, $-252.87^{\circ} \mathrm{C}$ ). There is no ideal working pair for adsorption cooling applications, but every system has an advantage over the other; therefore, the working pair should be chosen based on the application, from the point of interest of low evaporator temperature, or low driving temperature, or high SCP, or high COP. 
Table 9. Sorbent and system properties and performance

\begin{tabular}{|c|c|c|c|c|c|c|c|c|c|}
\hline $\begin{array}{c}\text { Sorption } \\
\text { pair/syste } \\
\text { m } \\
\end{array}$ & COP & $\mathrm{SCP}(\mathrm{W} / \mathrm{kg})$ & $\operatorname{Te}\left({ }^{\circ} \mathrm{C}\right)$ & $\operatorname{Td}\left({ }^{\circ} \mathrm{C}\right)$ & $\begin{array}{c}\text { Thermal } \\
\text { conductivity } \\
(W / \mathbf{m} \cdot \mathbf{k g}) \\
\end{array}$ & $\begin{array}{c}\text { Bulk density } \\
\left(\mathrm{kg} / \mathrm{m}^{3}\right)\end{array}$ & Characteristics & Comments & Reference \\
\hline \multirow{23}{*}{$\begin{array}{l}\text { Silica } \\
\text { gel/water }\end{array}$} & 0.61 & 208 & 12 & 82 & --- & --- & --- & --- & {$[22,23,133-137]$} \\
\hline & 0.4 & 85 & 10 & --- & --- & --- & Split heat pipe type evaporator & $\begin{array}{l}\text { Data obtained } \\
\text { experimentally }\end{array}$ & {$[22,109,138]$} \\
\hline & 0.36 & --- & 10 & 55 & --- & --- & --- & --- & {$[26,139]$} \\
\hline & 0.5 & --- & 15 & 85 & --- & --- & --- & --- & {$[26,140]$} \\
\hline & 0.50 & --- & 10 & 84 & --- & --- & --- & --- & {$[26,141]$} \\
\hline & 0.41 & --- & 2 & 80 & --- & --- & $\mathrm{x}_{\text {weak }}=0.03 \mathrm{~kg} / \mathrm{kg} ; \mathrm{x}_{\text {strong }}=0.125 \mathrm{~kg} / \mathrm{kg}$ & Data calculated & [26] \\
\hline & 0.31 & --- & 2 & 95 & --- & --- & $\mathrm{X}_{\text {weak }}=0.03 \mathrm{~kg} / \mathrm{kg} ; \mathrm{x}_{\text {strong }}=0.0 .80 \mathrm{~kg} / \mathrm{kg}$ & Data calculated & [26] \\
\hline & 0.26 & --- & 2 & 110 & --- & -- & $\mathrm{X}_{\text {weak }}=0.03 \mathrm{~kg} / \mathrm{kg} ; \mathrm{x}_{\text {strong }}=0.040 \mathrm{~kg} / \mathrm{kg}$ & Data calculated & {$[26]$} \\
\hline & --- & $2800 \mathrm{~kJ} / \mathrm{kg}$ & --- & --- & --- & 1000 & Used mostly for descent cooling & --- & [5] \\
\hline & $\begin{array}{l}0.20- \\
0.30\end{array}$ & --- & --- & --- & --- & --- & $\begin{array}{l}\text { Solar adsorption with } 170 \mathrm{~m}^{2} \text { of vacuum tube } \\
\text { collector }\end{array}$ & --- & {$[5,142]$} \\
\hline & $\begin{array}{ll}0.10- \\
0.13\end{array}$ & --- & --- & --- & --- & --- & -- & --- & {$[5,143]$} \\
\hline & 0.25 & --- & 14 & 55 & --- & --- & $\begin{array}{l}\text { Amount of adsorbent is allocated to adsorbent beds } \\
\text { and effect of mass ration is investigated }\end{array}$ & --- & {$[21,144]$} \\
\hline & $0.3-0.65$ & --- & 14 & 60 & --- & --- & Effect of silica gel mass on COP is investigated & --- & {$[21,145]$} \\
\hline & --- & --- & 5 & 100 & --- & --- & $\begin{array}{l}\text { Solar energy driven adsorption heat pumps are } \\
\text { studied }\end{array}$ & --- & {$[21,146]$} \\
\hline & 0.6 & --- & --- & --- & --- & --- & $\begin{array}{l}\text { AHP system is applied on air-conditioning of } \\
\text { buildings }\end{array}$ & --- & {$[21,147]$} \\
\hline & 0.5 & --- & -10 & 100 & --- & --- & Lab scale AHP system is constructed & --- & {$[21,148]$} \\
\hline & 0.6 & --- & 10 & 80 & --- & --- & $\begin{array}{l}\text { AHP system is applied on air-conditioning of } \\
\text { buildings }\end{array}$ & --- & {$[21,149]$} \\
\hline & 0.5 & --- & 10 to 20 & $80-95$ & --- & --- & $\begin{array}{l}\text { Small capacity AHP is constructed and tested for } \\
\text { heating and cooling applications }\end{array}$ & --- & {$[21,150]$} \\
\hline & --- & --- & 15 to 20 & 95 & --- & --- & $\begin{array}{l}\text { Compact solid sorption heat pump is developed and } \\
\text { tested }\end{array}$ & --- & {$[21,151]$} \\
\hline & $\begin{array}{ll}0.117- \\
0.143\end{array}$ & --- & 14 & $55-65$ & --- & --- & $\begin{array}{l}\text { Development of hybrid desiccant cooling system } \\
\text { combined with two stage adsorption chiller }\end{array}$ & --- & {$[21,152]$} \\
\hline & $0.32-0.4$ & --- & 15.1 & $55-67$ & --- & --- & Novel adsorption chiller is developed and tested & --- & {$[21,153]$} \\
\hline & 0.4 & --- & 15 & $60-92$ & --- & --- & $\begin{array}{l}\text { Micro adsorption chiller is applied on natural gas and } \\
\text { LPG power cogeneration system }\end{array}$ & --- & {$[21,154]$} \\
\hline & $0.427-$ & --- & --- & --- & --- & --- & Effects of variation of heat source on COP of AHP & --- & {$[21,155]$} \\
\hline
\end{tabular}




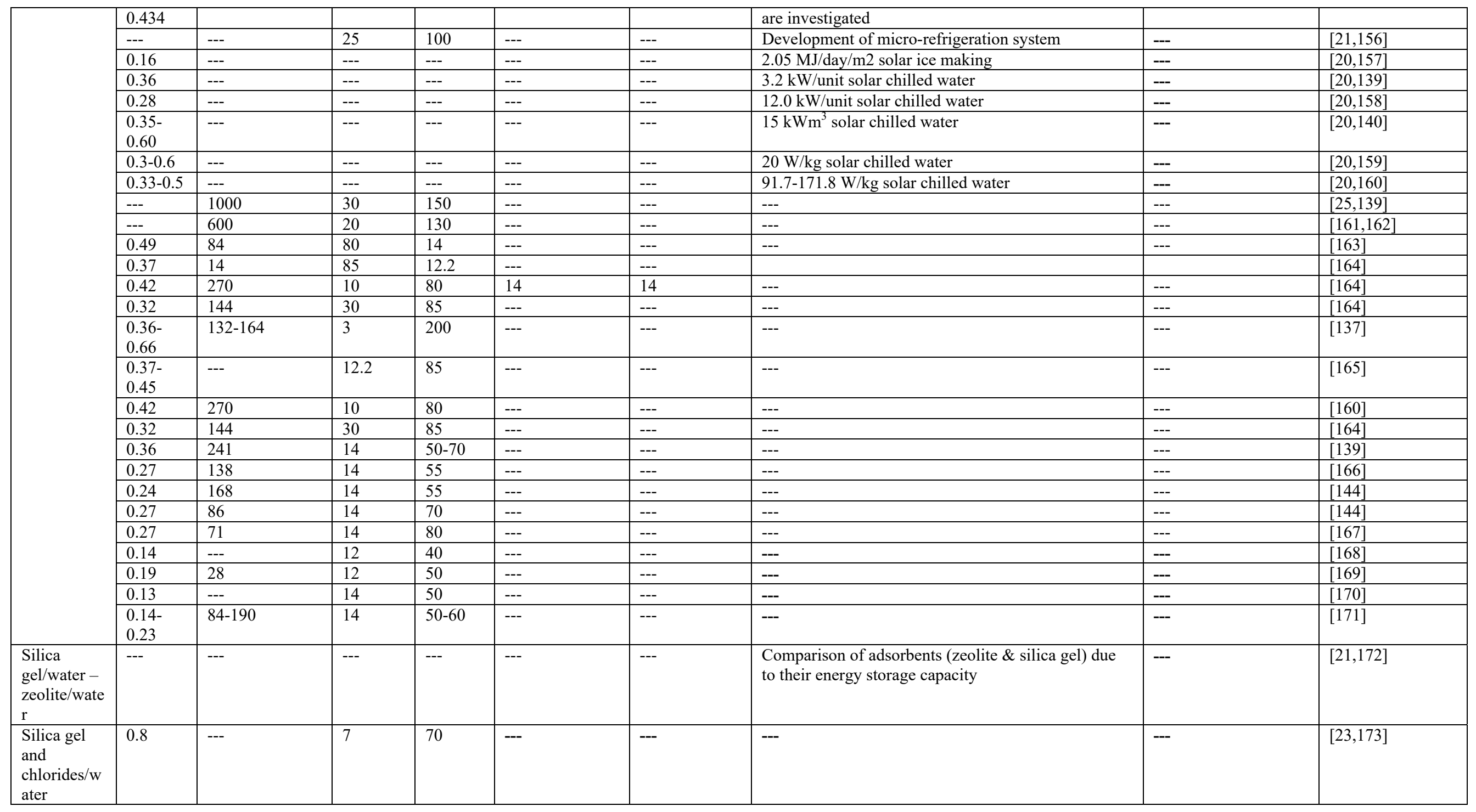




\begin{tabular}{|c|c|c|c|c|c|c|c|c|c|}
\hline $\begin{array}{l}\text { Silica gel- } \\
\mathrm{CaCl} 2 / \text { wate } \\
\mathrm{r}\end{array}$ & 0.30 & 20 & 150 & --- & --- & --- & --- & --- & {$[26,159]$} \\
\hline \multirow{2}{*}{$\begin{array}{l}\text { Silica } \\
\text { gel/methan } \\
\text { ol }\end{array}$} & --- & $\begin{array}{l}1000-1500 \\
\mathrm{~kJ} / \mathrm{kg}\end{array}$ & --- & --- & --- & 791 & Suitable for temperature less than $200^{\circ} \mathrm{C}$ & --- & {$[5,17]$} \\
\hline & 0.3 & --- & 0 & 85 & --- & --- & $\begin{array}{l}\text { Solar-hybrid powered AHP system is designed and } \\
\text { applied for decentralized cold storage of agricultural } \\
\text { products }\end{array}$ & --- & {$[21,174]$} \\
\hline $\begin{array}{l}\text { Silica gel } \\
\text { and } \\
\text { chlorides/m } \\
\text { ethanol }\end{array}$ & 0.33 & --- & -10 & 47 & --- & --- & --- & --- & {$[23,175,176]$} \\
\hline \multirow{2}{*}{$\begin{array}{l}\text { Silica } \\
\text { gel/air }\end{array}$} & 0.935 & --- & 29 & 90 & --- & --- & --- & --- & {$[26,177]$} \\
\hline & 0.935 & --- & 25 & 80 & --- & --- & --- & --- & {$[26,48]$} \\
\hline $\begin{array}{l}\text { Activated } \\
\text { alumina/wa } \\
\text { ter }\end{array}$ & --- & $3000 \mathrm{~kJ} / \mathrm{kg}$ & --- & --- & --- & 1000 & $\begin{array}{l}\text { Water is applicable except for very low operation } \\
\text { pressure }\end{array}$ & --- & {$[5,17]$} \\
\hline \multirow{12}{*}{$\begin{array}{l}\text { Zeolite/wat } \\
\text { er }\end{array}$} & 0.4 & 600 & 6.5 & 350 & --- & --- & --- & --- & {$[23,178-181]$} \\
\hline & 0.9 & $125^{*}$ & 5 & --- & $\begin{array}{c}--- \\
\end{array}$ & --- & Intermittent convective thermal wave cycle & Data calculated & {$[22,182]$} \\
\hline & --- & $\begin{array}{l}3300-4200 \\
\mathrm{~kJ} / \mathrm{kg}\end{array}$ & -- & --- & --- & 1000 & Natural zeolite as lower values than synthetic zeolite & --- & {$[5,17]$} \\
\hline & $\begin{array}{l}0.10- \\
0.12\end{array}$ & --- & --- & --- & --- & --- & Solar adsorption with $1.5 \mathrm{~m}^{2}$ of flat plate collector & --- & {$[5,184,185]$} \\
\hline & 0.34 & --- & 27 & 123 & --- & --- & $\begin{array}{l}\text { Natural zeolite seem as alternative adsorbent for } \\
\text { adsorption heat pump system }\end{array}$ & --- & {$[21,186]$} \\
\hline & 0.34 & --- & 25 & 200 & --- & --- & $\begin{array}{l}\text { Natural zeolite seem as alternative adsorbent for } \\
\text { adsorption heat pump system }\end{array}$ & --- & {$[21,187]$} \\
\hline & 0.74 & ---- & 4 & 230 & $\begin{array}{c}--- \\
\end{array}$ & --- & The bed design enhanced mass and heat transfer rate & --- & {$[21,188]$} \\
\hline & $\begin{array}{l}\text { Single } \\
0.3- \\
0.38, \\
\text { double } \\
0.5-0.73 \\
\end{array}$ & --- & 3 & $120-360$ & --- & --- & $\begin{array}{l}\text { Effect of regeneration system temperature on COP of } \\
\text { system }\end{array}$ & --- & {$[21,181]$} \\
\hline & --- & --- & 3 & 290 & --- & --- & $\begin{array}{l}\text { Influence of physical properties of heat transfer fluid } \\
\text { on AHP system }\end{array}$ & --- & {$[21,189]$} \\
\hline & --- & --- & 3 & 210 & --- & --- & Optimum coating thickness is determined & --- & {$[21,190]$} \\
\hline & 0.3 & --- & 2 & 150 & --- & -- & $\begin{array}{l}\text { Lighter adsorption heat pump system can be obtained } \\
\text { with using polymeric heat exchanger tubes }\end{array}$ & --- & {$[21,191]$} \\
\hline & $0.94-$ & --- & 0 & 350 & -- & --- & Exergy analysis of four cascades cycled heat pump & --- & {$[21,192]$} \\
\hline
\end{tabular}




\begin{tabular}{|c|c|c|c|c|c|c|c|c|c|}
\hline & 1.008 & & & & & & systems is performed & & \\
\hline & 0.46 & --- & 7 & 200 & --- & $-\overline{--}$ & $\begin{array}{l}\text { Open cell cooper foam is applied on AHP for } \\
\text { improving heat transfer properties }\end{array}$ & --- & {$[21,193]$} \\
\hline & --- & --- & --- & --- & --- & --- & $\begin{array}{l}\text { Optimization of zeolite coating process on stainless } \\
\text { steel tube }\end{array}$ & --- & {$[21,194]$} \\
\hline & --- & --- & 2 & 135 & --- & --- & Optimum coating thickness is determined & --- & {$[21,196]$} \\
\hline & --- & --- & 2 & 151 & --- & --- & $\begin{array}{l}\text { The effects of thermal and mass diffusivities on the } \\
\text { performance of AHP is studied }\end{array}$ & --- & {$[21,197]$} \\
\hline & --- & --- & --- & --- & --- & --- & Solar energy driven AHP is designed & --- & {$[21,199]$} \\
\hline & --- & --- & 2 & --- & --- & --- & Solar powered AHP is used for ice-making & --- & {$[21,200]$} \\
\hline & --- & --- & 2 & 151 & --- & --- & Effect of metal mass on the performance of AHP & --- & {$[21,201]$} \\
\hline & 1.4 & --- & --- & 96 & --- & --- & $\begin{array}{l}\text { AHP system is applied on air-conditioning of } \\
\text { buildings }\end{array}$ & --- & {$[21,202]$} \\
\hline & $\begin{array}{l}0.43- \\
1.04 \\
\end{array}$ & --- & 10 to 40 & 150 & --- & --- & One of the first applications & --- & {$[21,203]$} \\
\hline & $0.6-1.6$ & --- & --- & --- & --- & --- & 36-144 W/kg solar air conditioning & $\begin{array}{ll}-- \\
\end{array}$ & {$[20,204]$} \\
\hline & $\begin{array}{l}0.20- \\
0.21 \\
\end{array}$ & --- & --- & --- & --- & --- & $21.4-30 \mathrm{~W} / \mathrm{kg}$ solar air conditioning & --- & {$[20,207]$} \\
\hline & 0.44 & 48.8 & 6 & 200 & --- & --- & --- & --- & [208] \\
\hline $\begin{array}{l}\text { Zeolite } \\
\text { NaX/water }\end{array}$ & $\begin{array}{l}0.39- \\
1.03 \\
\end{array}$ & --- & 3 & 200 & --- & --- & --- & --- & [181] \\
\hline & $\begin{array}{l}0.44- \\
0.47\end{array}$ & $48.8-52$ & 6 & 200 & --- & --- & --- & --- & [209] \\
\hline $\begin{array}{l}\text { Zeolite/wat } \\
\text { er/carbon/m } \\
\text { ethanol }\end{array}$ & 1.06 & --- & 25 & $\begin{array}{l}220 \text { for } \\
\text { zeolite } \\
\text { bed, } \\
100 \text { for } \\
\text { carbon } \\
\text { bed }\end{array}$ & $\overline{---}$ & $\begin{array}{l}-- \\
\end{array}$ & $\begin{array}{l}\text { Heat required during regeneration of carbon bed is } \\
\text { provided from zeolite beds }\end{array}$ & $\begin{array}{l}-- \\
\end{array}$ & {$[21,161]$} \\
\hline $\begin{array}{l}\text { Zeolite and } \\
\text { foam } \\
\text { aluminium/ } \\
\text { water }\end{array}$ & 0.55 & 500 & 10 & 250 & --- & --- & --- & --- & {$[23,162]$} \\
\hline
\end{tabular}




\begin{tabular}{|c|c|c|c|c|c|c|c|c|c|}
\hline $\begin{array}{l}\text { Zeolite/am } \\
\text { monia }\end{array}$ & --- & $\begin{array}{l}4000-6000 \\
\mathrm{~kJ} / \mathrm{kg}\end{array}$ & --- & --- & --- & 681 & --- & --- & {$[5]$} \\
\hline \multirow[t]{2}{*}{$\begin{array}{l}\text { Zeolite/met } \\
\text { hanol }\end{array}$} & --- & $\begin{array}{l}2300-2600 \\
\mathrm{~kJ} / \mathrm{kg}\end{array}$ & --- & $\begin{array}{ll}-- \\
-1\end{array}$ & --- & 791 & --- & --- & {$[5]$} \\
\hline & 1.34 & --- & -5 & 155 & --- & --- & Lab apparatus is constructed. & --- & {$[21,210]$} \\
\hline Zeolite 4A & --- & $1250 \mathrm{~kJ} / \mathrm{kg}$ & 30 & 350 & --- & --- & --- & --- & {$[25,139]$} \\
\hline Zeolite 5A & --- & $1200 \mathrm{~kJ} / \mathrm{kg}$ & 30 & 350 & --- & --- & --- & --- & {$[25,139]$} \\
\hline \multirow[t]{3}{*}{$\begin{array}{l}\text { Zeolite } \\
13 \mathrm{X} / \text { water }\end{array}$} & --- & --- & 10 & 150 & --- & --- & $\begin{array}{l}\text { Polyaniline/zeolite composite is prepared for } \\
\text { enhancement of heat transfer of adsorbent bed }\end{array}$ & --- & {$[21,211]$} \\
\hline & --- & $1290 \mathrm{~kJ} / \mathrm{kg}$ & 30 & 350 & --- & --- & --- & --- & {$[25,139]$} \\
\hline & --- & $930 \mathrm{~kJ} / \mathrm{kg}$ & 20 & 300 & --- & --- & --- & --- & {$[25,139]$} \\
\hline $\begin{array}{l}\text { Zeolite } \\
\text { MgA }\end{array}$ & --- & 800 & 60 & 250 & --- & --- & --- & --- & {$[25,139]$} \\
\hline Zeolite/ $\mathrm{CO}_{2}$ & --- & --- & 0 & -78.15 & --- & --- & --- & --- & {$[212,213]$} \\
\hline Zeolite $/ \mathrm{N}_{2}$ & --- & --- & 0 & -195.8 & --- & --- & --- & --- & {$[212]$} \\
\hline $\begin{array}{l}\text { Zeolite } \\
\text { 13X- } \\
\text { LiBr/water }\end{array}$ & 0.33 & --- & 12 to 6 & 110 & --- & --- & $\begin{array}{l}\text { Zeolite/water adsorption system combined } \\
\mathrm{LiBr} / \text { water absorption system for providing } \\
\text { continuous system }\end{array}$ & --- & {$[21,214]$} \\
\hline $\begin{array}{l}\text { Graphite } \\
\text { foams }\end{array}$ & --- & --- & --- & --- & $100-150$ & --- & --- & --- & {$[9,215]$} \\
\hline AC/water & 0.05 & --- & --- & --- & --- & --- & Solar adsorption with $2 \mathrm{~m}^{2}$ of flat plate collector & --- & {$[5,216]$} \\
\hline \multirow{11}{*}{$\begin{array}{l}\text { AC/methan } \\
\text { ol }\end{array}$} & 0.114 & --- & --- & --- & ---- & --- & Adsorptive solar refrigeration system is simulated & --- & {$[21,217]$} \\
\hline & 0.12 & --- & -10 & 100 & --- & --- & Solar powered AHP is used for ice-making & --- & {$[21,218]$} \\
\hline & 0.12 & --- & --- & --- & --- & --- & $6 \mathrm{~kg} /$ day $/ \mathrm{m} 2$ solar ice making & --- & {$[20,219]$} \\
\hline & --- & $\begin{array}{ll}-- \\
-1\end{array}$ & --- & --- & --- & --- & $\begin{array}{l}\text { Dynamic simulation of AHP is studied with zeolite } \\
\text { coated adsorbent bed }\end{array}$ & --- & {$[21,220]$} \\
\hline & & 590 & 20 & 140 & & & --- & --- & {$[25,139]$} \\
\hline & 0.5 & -10 & 100 & --- & --- & --- & Dynamic analysis of heat recovery process for AHP & --- & {$[21,221]$} \\
\hline & $\begin{array}{ll}0.12- \\
0.14\end{array}$ & --- & $\begin{array}{ll}--- \\
\end{array}$ & $\begin{array}{ll}--- \\
\end{array}$ & --- & --- & $5-6 \mathrm{~kg} /$ day $/ \mathrm{m} 2$ solar ice making & --- & {$[20,222]$} \\
\hline & $\begin{array}{l}0.13- \\
0.15\end{array}$ & --- & --- & --- & --- & --- & $6-7 \mathrm{~kg} / \mathrm{day} / \mathrm{m} 2$ solar ice making & --- & {$[20,223]$} \\
\hline & 0.18 & --- & --- & --- & --- & --- & $27 \mathrm{~W} / \mathrm{kg}$ solar ice making & --- & {$[20,224]$} \\
\hline & 0.4 & $\begin{array}{ll}-- \\
\end{array}$ & --- & $\begin{array}{c}-- \\
\end{array}$ & --- & --- & 73.1 W/unit solar chilled water & --- & {$[20,225]$} \\
\hline & 0.47 & 148 & 10 & 100 & --- & --- & --- & --- & {$[209,226]$} \\
\hline $\begin{array}{l}\text { AC+blacke } \\
\text { ned } \\
\text { steel/metha } \\
\text { nol }\end{array}$ & 0.16 & $\begin{array}{ll}--- \\
\end{array}$ & --- & $\begin{array}{ll}--- \\
\end{array}$ & --- & --- & $9.4 \mathrm{~kg} /$ day $/ \mathrm{m} 2$ solar ice making & --- & {$[20,227]$} \\
\hline
\end{tabular}




\begin{tabular}{|c|c|c|c|c|c|c|c|c|c|}
\hline \multirow{7}{*}{$\begin{array}{l}\text { AC/ammon } \\
\text { ia }\end{array}$} & 0.61 & 2000 & -5 & 100 & --- & --- & --- & --- & {$[23,106,228-232]$} \\
\hline & --- & 1000 & 8 & --- & --- & $\begin{array}{ll}-- \\
--\end{array}$ & Convective thermal wave cycle & Data calculated & {$[22,101]$} \\
\hline & 0.67 & 557 & 3 & --- & --- & --- & Convective thermal wave cycle & Data calculated & {$[22,234]$} \\
\hline & $\begin{array}{l}\text { Cooling } \\
0.36, \\
\text { heating } \\
1.1-1.15\end{array}$ & --- & --- & --- & --- & --- & $\begin{array}{l}\text { Two ton solid sorption heat pump system is built and } \\
\text { tested for cooling and heating }\end{array}$ & --- & {$[21,235]$} \\
\hline & 0.05 & --- & --- & --- & --- & --- & Solar adsorption with $1 \mathrm{~m}^{2}$ of flat plate collector & --- & {$[5,236]$} \\
\hline & $\begin{array}{l}0.42- \\
1.19\end{array}$ & --- & --- & --- & --- & --- & Solar air conditioning & --- & {$[20,237]$} \\
\hline & 0.10 & --- & --- & --- & --- & --- & $35 \mathrm{~W} / \mathrm{kg}$ solar ice making & --- & {$[20,229]$} \\
\hline \multirow{10}{*}{$\begin{array}{l}\mathrm{AC}- \\
\mathrm{CaCl} 2 / \mathrm{amm} \\
\text { onia }\end{array}$} & 0.41 & 731 & -15 & --- & --- & --- & $\begin{array}{l}\text { Composite adsorbent, heat pipe type heating and } \\
\text { cooling }\end{array}$ & $\begin{array}{l}\text { Data obtained } \\
\text { experimentally }\end{array}$ & {$[22,114,239]$} \\
\hline & 0.36 & 627.7 & -25 & --- & --- & $\begin{array}{ll}-- \\
-\end{array}$ & $\begin{array}{l}\text { Composite adsorbent, heat pipe type heating and } \\
\text { cooling }\end{array}$ & $\begin{array}{l}\text { Data obtained } \\
\text { experimentally }\end{array}$ & {$[22,114,239]$} \\
\hline & --- & --- & --- & --- & --- & --- & $\begin{array}{l}\text { Ammonia adsorption behaviour on new adsorbent } \\
\text { composite materials }\end{array}$ & --- & {$[21,240]$} \\
\hline & 0.36 & --- & -20 & 135 & --- & $\begin{array}{ll}-- \\
\end{array}$ & $\begin{array}{l}\text { Ice-making system uses heat pipe principle to } \\
\text { perform the heating and cooling process for adsorber }\end{array}$ & --- & {$[21,241]$} \\
\hline & 0.39 & --- & --- & --- & --- & --- & $770 \mathrm{~W} / \mathrm{kg}$ solar ice making & --- & {$[20,243]$} \\
\hline & 0.26 & 528 & -19 & 114 & --- & --- & --- & --- & {$[243]$} \\
\hline & 0.23 & 409.1 & -18.9 & 160 & --- & --- & --- & --- & {$[244,245]$} \\
\hline & 0.26 & 528 & -10 & 145 & --- & --- & --- & --- & [245] \\
\hline & $\begin{array}{l}0.23- \\
0.27\end{array}$ & $409.1-486.5$ & -18.9 & 160 & --- & --- & --- & --- & [244] \\
\hline & 0.14 & --- & -19.7 & 103 & --- & --- & --- & --- & {$[246]$} \\
\hline $\begin{array}{l}\text { AC fiber- } \\
\mathrm{CaCl} 2 / \mathrm{amm} \\
\text { onia }\end{array}$ & 0.6 & 330 & 1 & --- & --- & --- & Composite adsorbent, heat pipe heating & $\begin{array}{l}\text { Data obtained } \\
\text { experimentally }\end{array}$ & {$[22,122]$} \\
\hline $\mathrm{AC} / \mathrm{H}_{2}$ & --- & ---- & 30 & -252.87 & --- & $\begin{array}{ll}-- \\
\end{array}$ & --- & ---- & {$[247,248]$} \\
\hline $\begin{array}{l}\mathrm{AC} / \mathrm{HFC} \\
\text { refrigerant }\end{array}$ & 0.43 & --- & -60 & 150 & --- & --- & $\begin{array}{l}\text { System requires operating pressure about } 8 \text { bars for } \\
\text { HFC as adsorbate }\end{array}$ & --- & {$[21,249]$} \\
\hline $\begin{array}{l}\mathrm{CaCl} 2 / \text { meth } \\
\text { anol }\end{array}$ & --- & $\begin{array}{l}1800-2000 \\
\mathrm{~kJ} / \mathrm{kg}\end{array}$ & --- & --- & --- & 791 & Used for cooling & --- & [5] \\
\hline $\begin{array}{l}\mathrm{SrCl} 2 / \mathrm{amm} \\
\text { onia }\end{array}$ & 0.32 & 230 & -10 & --- & --- & --- & Single-effect system & $\begin{array}{l}\text { Data obtained } \\
\text { experimentally }\end{array}$ & {$[22,250]$} \\
\hline
\end{tabular}




\begin{tabular}{|c|c|c|c|c|c|c|c|c|c|}
\hline $\begin{array}{l}(\mathrm{MnCl} 2- \\
\mathrm{NiCl} 2) / \mathrm{am} \\
\text { monia }\end{array}$ & 0.4 & $70^{*}$ & -25 & --- & --- & $\begin{array}{ll}-- \\
\end{array}$ & Double-effect system & Data calculated & {$[22,250]$} \\
\hline \multirow{5}{*}{$\begin{array}{l}\text { AC/methan } \\
\text { ol }\end{array}$} & 0.78 & 16 & 15 & 90 & --- & --- & --- & --- & {$[23,251-253]$} \\
\hline & $\begin{array}{l}0.10- \\
0.12\end{array}$ & --- & -3 & 110 & --- & --- & Solar adsorption with $6 \mathrm{~m}^{2}$ of flat plate collector & --- & {$[5,184]$} \\
\hline & $\begin{array}{l}0.10- \\
0.12\end{array}$ & --- & -6 & $70-78$ & --- & $\begin{array}{ll}-- \\
-\end{array}$ & Solar adsorption with $6 \mathrm{~m}^{2}$ of flat plate collector & --- & {$[5,17]$} \\
\hline & 0.38 & --- & $\begin{array}{ll}-20 & \text { to } \\
+15 & \end{array}$ & $40-100$ & --- & --- & $\begin{array}{l}\text { Mass transfer is enhanced by using activated carbon } \\
\text { fibre }\end{array}$ & --- & {$[21,254]$} \\
\hline & $\begin{array}{l}0.55- \\
0.61\end{array}$ & $140-190$ & 40 & $140-150$ & --- & --- & --- & --- & [136] \\
\hline AC/ethanol & 0.8 & --- & 3 & 80 & --- & --- & --- & --- & {$[23,256,257]$} \\
\hline $\mathrm{AC} / \mathrm{N}_{2}$ & --- & --- & 30 & $\begin{array}{l}-195.8 \\
\end{array}$ & --- & --- & --- & --- & {$[258]$} \\
\hline $\begin{array}{l}\text { Consolidate } \\
\text { d zeolite 4A }\end{array}$ & --- & 312 & --- & --- & 0.3 & 960 & --- & --- & {$[9,260,261]$} \\
\hline $\begin{array}{l}\text { Consolidate } \\
\mathrm{d} \text { zeolite } 4 \mathrm{~A} \\
+ \text { adhesive } \\
\text { bond to } \\
\text { wall }\end{array}$ & --- & 432 & --- & --- & 0.3 & 960 & --- & --- & {$[9,260,261]$} \\
\hline $\begin{array}{l}\text { Consolidate } \\
\mathrm{d} \quad \text { zeolite } \\
13 \mathrm{X}\end{array}$ & --- & --- & --- & --- & 0.58 & --- & --- & --- & {$[9,262]$} \\
\hline $\begin{array}{l}\text { Consolidate } \\
\text { d carbon }\end{array}$ & --- & 16 & --- & --- & $0.27-0.34$ & --- & --- & --- & {$[9,253]$} \\
\hline $\begin{array}{l}\text { Consolidate } \\
\mathrm{d} \\
\text { carbon/carb } \\
\text { on fibre + } \\
\text { aluminium } \\
\text { fins }\end{array}$ & --- & 446 & --- & --- & --- & --- & --- & --- & {$[9,263]$} \\
\hline $\begin{array}{l}\text { Consolidate } \\
\mathrm{d} \\
\text { carbon/met } \\
\text { hanol }\end{array}$ & 0.1 & 13 & --- & --- & --- & --- & --- & --- & {$[9,264]$} \\
\hline $\begin{array}{l}\text { Consolidate } \\
\text { d }\end{array}$ & 1.4 & 400 & --- & --- & --- & $\begin{array}{l}-- \\
\end{array}$ & --- & --- & {$[9,265]$} \\
\hline
\end{tabular}




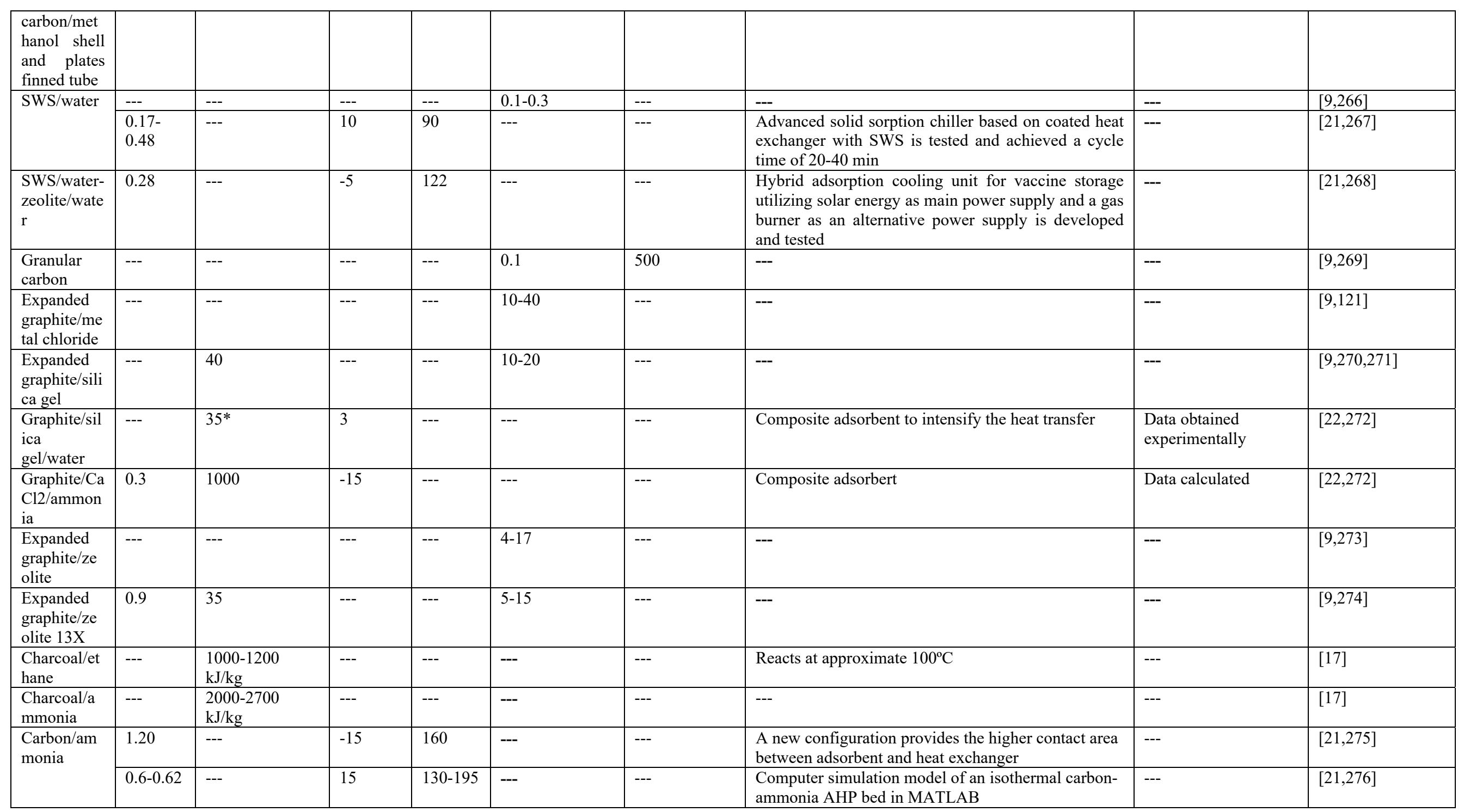




\begin{tabular}{|c|c|c|c|c|c|c|c|c|c|}
\hline & 0.3 & --- & 9.5 & 150 & --- & --- & $\begin{array}{l}\text { Experimental test of compact sorption generator } \\
\text { prototype }\end{array}$ & $\begin{array}{l}-- \\
\end{array}$ & {$[21,277]$} \\
\hline $\begin{array}{l}\text { Charcoal } / \mathrm{w} \\
\text { ater }\end{array}$ & --- & $\begin{array}{l}2300-2700 \\
\mathrm{~kJ} / \mathrm{kg}\end{array}$ & --- & --- & --- & --- & --- & --- & [17] \\
\hline $\begin{array}{l}\text { Carbon/wat } \\
\text { er }\end{array}$ & 0.95 & --- & 0 & 250 & --- & --- & $\begin{array}{l}\text { Forced convection is used for improving mass } \\
\text { transfer }\end{array}$ & --- & {$[21,278]$} \\
\hline $\begin{array}{l}\text { Charcoal } / \mathrm{m} \\
\text { ethanol }\end{array}$ & --- & $\begin{array}{l}1800-2000 \\
\mathrm{~kJ} / \mathrm{kg}\end{array}$ & $\begin{array}{l}-- \\
\end{array}$ & --- & --- & --- & --- & $\begin{array}{l}-- \\
\end{array}$ & [17] \\
\hline $\begin{array}{l}\text { Carbon } / \text { met } \\
\text { hanol }\end{array}$ & --- & --- & 9 & 110 & --- & --- & $\begin{array}{l}\text { The system is driven by waste heat exhausted from } \\
\text { engine }\end{array}$ & --- & {$[21,279]$} \\
\hline $\begin{array}{l}\text { Carbon- } \\
\text { methanol/ze } \\
\text { olite/water } \\
\text { carbon- } \\
\text { ammonia }\end{array}$ & --- & --- & --- & --- & --- & --- & $\begin{array}{l}\text { Common used adsorbent-adsorbate pairs are } \\
\text { investigaged }\end{array}$ & --- & {$[21,281]$} \\
\hline $\begin{array}{l}\text { Charcoal/et } \\
\text { hanol }\end{array}$ & --- & $\begin{array}{l}1200-1400 \\
\mathrm{~kJ} / \mathrm{kg}\end{array}$ & $\begin{array}{l}-- \\
\end{array}$ & --- & --- & --- & --- & $\begin{array}{l}-- \\
\end{array}$ & {$[17]$} \\
\hline $\begin{array}{l}\text { Carbon } \\
\text { fiber/ethano } \\
1\end{array}$ & --- & --- & --- & 80 & --- & --- & $\begin{array}{l}\text { Adsorbent bed generally has heavy weight. Adsorber } \\
\text { must be designed lightweight to reduce sensible heat } \\
\text { losses from body }\end{array}$ & --- & {$[21,282]$} \\
\hline \multirow{3}{*}{$\begin{array}{l}\text { Monolithic } \\
\text { carbon }\end{array}$} & --- & --- & --- & --- & 0.6 & 750 & --- & --- & {$[9,283]$} \\
\hline & --- & --- & --- & --- & 0.33 & 713 & --- & --- & {$[9,284]$} \\
\hline & --- & --- & --- & --- & $0.27-0.34$ & --- & --- & --- & {$[9,253,285]$} \\
\hline $\begin{array}{l}\text { Monolithic } \\
\text { carbon/plat } \\
\text { e heat } \\
\text { exchanger } \\
\text { (simulation) }\end{array}$ & --- & 2000 & --- & --- & --- & --- & --- & --- & {$[9,106]$} \\
\hline $\begin{array}{l}\text { Monolithic } \\
\text { carbon/alu } \\
\text { minium } \\
\text { laminate }\end{array}$ & --- & 200 & --- & --- & 20 & --- & --- & --- & {$[9,286]$} \\
\hline $\begin{array}{l}\text { Carbon } \\
\text { matrix } \\
\text { (carbide } \\
\text { precursor) }\end{array}$ & --- & --- & --- & --- & $2-10$ & --- & --- & $\begin{array}{l}-- \\
\end{array}$ & {$[9,287]$} \\
\hline $\begin{array}{l}\text { Metal } \\
\text { chloride/am }\end{array}$ & 0.6 & --- & -10 & 52 & --- & --- & --- & --- & {$[23,288,289]$} \\
\hline
\end{tabular}




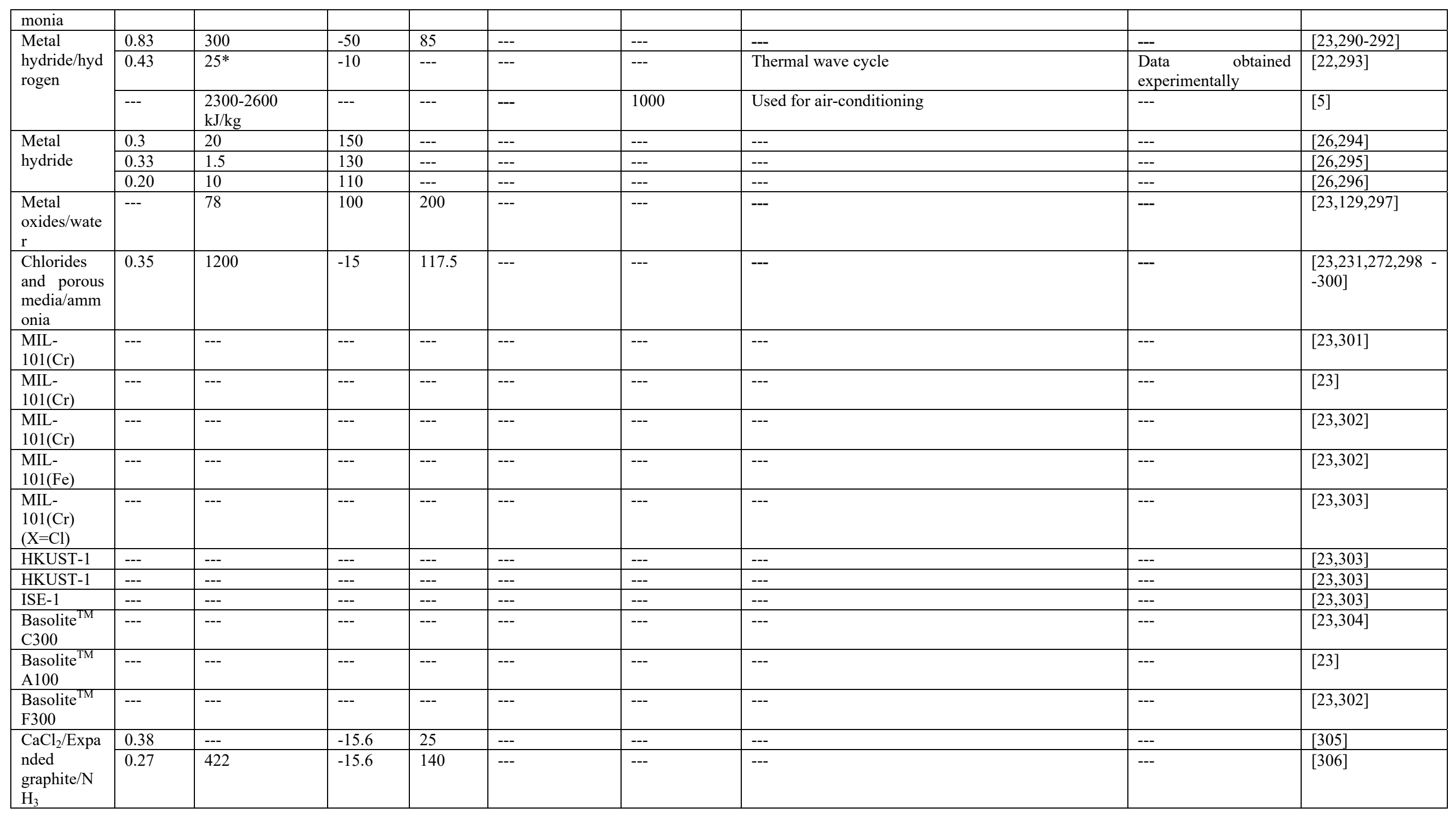






*The SCP in the original literature was calculated based on the total cycle time, with adsorption and desorption of same length. To allow a fair comparison, the original value should be multiplied by two. 
Table 9 shows the newly presented adsorbent-refrigerant pair, developed to achieve optimized adsorption working systems. Among of these pairs, AC/R134a has a relatively high adsorption capacity and low evaporation temperature $\left(-26.55^{\circ} \mathrm{C}\right)$. But the problem with $\mathrm{R} 134 \mathrm{a}$ that it has a high global worming potential value (1300). The lowest adsorption capacity is for the pair of $\mathrm{AC} / \mathrm{N}_{2}$ and the highest one is for the pair of $\mathrm{AC} / \mathrm{R} 134 \mathrm{a}$. Although the $\mathrm{AC} / \mathrm{N}_{2}$ has a relatively low adsorption capacity, it has the lowest boiling temperature $\left(-195.8^{\circ} \mathrm{C}\right)$ which means it will be good to be used in refrigeration applications. AC/diethyl ether has a relatively high adsorption capacity of about 1.63 but it has the disadvantages of working at vacuum pressure and a relatively high boiling temperature of about $34{ }^{\circ} \mathrm{C}$.

Heat of adsorption can be derived from adsorption isotherms (Figure 4), referred to as isosteric heat (the energy released in the adsorption process), or as the differential heat of adsorption determined experimentally using a calorimetric method (Table 9) [17]. To understand the adsorption process, the adsorption equilibria are introduced to describe the adsorption process and several state equations known as isotherms of adsorption are proposed [318,319]. Several basic theories have been proposed and used to define the main isotherms of an adsorption process, the Henry's law [320], the Langmuir's approach [321], the Gibbs' theory, and the adsorption potential theory; other recent equations have been developed recently and are presented in Table 10. 
Table 10.Thermodynamic reaction values of common metal chloride/bromide/iodide-ammonia sorption pairs $[18,316,317]$

\begin{tabular}{|c|c|c|c|c|c|c|}
\hline Reactive salt & $\begin{array}{l}\text { Reaction } \\
\text { enthalpy } \\
(\mathrm{J} / \mathrm{mol})\end{array}$ & $\begin{array}{l}\text { Reaction } \\
\text { entropy } \\
(\mathrm{K} / \mathrm{mol} \mathrm{K})\end{array}$ & $\begin{array}{l}\text { Reaction } \\
\text { coefficient } m\end{array}$ & $\begin{array}{l}\text { Reaction } \\
\text { coefficient } n\end{array}$ & $\begin{array}{l}\text { Molar } \\
\text { mass } \\
\text { (g/mol) }\end{array}$ & $\begin{array}{l}\text { Driving } \\
\text { equilibrium } \\
\text { temperature } \\
\left(\mathrm{T}_{\mathrm{C}}=30^{\circ} \mathrm{C}\right)\end{array}$ \\
\hline $\mathrm{NH}_{4} \mathrm{Cl}$ & 29.433 & 207.9 & 3 & 0 & 53.4 & 48 \\
\hline KI & 32.015 & 219.8 & 4 & 0 & 166 & 36 \\
\hline $\mathrm{LiBr}$ & 33.689 & 225.9 & 1 & 4 & 86.8 & 34 \\
\hline $\mathrm{PbCl} 2$ & 34.317 & 223.6 & 4.75 & 3.25 & 278 & 47 \\
\hline $\mathrm{NaBr}$ & 35.363 & 225.2 & 5.25 & 0 & 102.9 & 51 \\
\hline $\mathrm{CaI}_{2}$ & 35.991 & 229.3 & 2 & 6 & 293.8 & 45 \\
\hline $\mathrm{LiCl}$ & 36.828 & 224.6 & 1 & 3 & 42.3 & 67 \\
\hline $\mathrm{PbBr}_{2}$ & 37.665 & 229.4 & 2.5 & 3 & 367 & 60 \\
\hline $\mathrm{BaCl}_{2}$ & 38.250 & 232.4 & 8 & 0 & 208.2 & 56 \\
\hline $\mathrm{PbCl}_{2}$ & 39.339 & 230.1 & 1.25 & 2 & 178 & 72 \\
\hline $\mathrm{PbBr}_{2}$ & 39.758 & 229.4 & 1 & 2 & 367 & 78 \\
\hline $\mathrm{PbI}_{2}$ & 40.595 & 229.1 & 3 & 2 & 461 & 87 \\
\hline $\mathrm{CaCl}_{2}$ & 41.013 & 230.1 & 4 & 4 & 110.8 & 87 \\
\hline $\mathrm{NaI}$ & 39.339 & 224.5 & 4.5 & 0 & 149.9 & 90 \\
\hline $\mathrm{BaBr}_{2}$ & 41.850 & 229.8 & 4 & 4 & 297.1 & 95 \\
\hline $\mathrm{SrCl}_{2}$ & 41.432 & 228.6 & 7 & 1 & 158.4 & 96 \\
\hline $\mathrm{CaCl}_{2}$ & 42.269 & 229.7 & 2 & 2 & 110.8 & 99 \\
\hline $\mathrm{BaBr}_{2}$ & 42.687 & 230.7 & 2 & 2 & 297 & 100 \\
\hline $\mathrm{LiCl}$ & 44.780 & 229.8 & 1 & 2 & 42.3 & 120 \\
\hline $\mathrm{SrBr}_{2}$ & 45.617 & 229.3 & 6 & 2 & 247.4 & 130 \\
\hline $\mathrm{BaI}_{2}$ & 46.454 & 231.6 & 2 & 4 & 391.1 & 130 \\
\hline $\mathrm{BaI}_{2}$ & 47.291 & 230.3 & 2 & 2 & 391.1 & 142 \\
\hline $\mathrm{MnCl}_{2}$ & 47.416 & 227.9 & 4 & 2 & 125.7 & 152 \\
\hline $\mathrm{LiCl}$ & 48.128 & 230.6 & 1 & 1 & 42.3 & 148 \\
\hline $\mathrm{CaBr}_{2}$ & 48.965 & 230.4 & 4 & 2 & 199.8 & 156 \\
\hline $\mathrm{ZnCl}_{2}$ & 49.467 & 230.0 & 2 & 2 & 136.2 & 162 \\
\hline $\mathrm{FeCl}_{2}$ & 54.266 & 227.8 & 4 & 2 & 126.6 & 186 \\
\hline $\mathrm{SrI}_{2}$ & 52.731 & 230.5 & 4 & 2 & 341.4 & 188 \\
\hline $\mathrm{MnBr}_{2}$ & 53.066 & 228.3 & 4 & 2 & 214.7 & 200 \\
\hline $\mathrm{CoCl}_{2}$ & 53.987 & 227.9 & 4 & 2 & 94.3 & 210 \\
\hline $\mathrm{MgCl}_{2}$ & 55.661 & 230.4 & 4 & 2 & 95.1 & 214 \\
\hline $\mathrm{FeBr}_{2}$ & 55.828 & 228.1 & 4 & 2 & 215.6 & 226 \\
\hline $\mathrm{BaI}_{2}$ & 56.079 & 235.0 & 2 & 0 & 391.1 & 199 \\
\hline $\mathrm{CaI}_{2}$ & 58.590 & 231. & 4 & 2 & 293.8 & 237 \\
\hline $\mathrm{CoBr}_{2}$ & 58.590 & 227.5 & 4 & 2 & 218.7 & 253 \\
\hline $\mathrm{NiCl}_{2}$ & 59.218 & 227.6 & 4 & 2 & 129.5 & 259 \\
\hline $\mathrm{MnI}_{2}$ & 59.301 & 227.4 & 4 & 2 & 308.7 & 260 \\
\hline $\mathrm{FeI}_{2}$ & 60.683 & 227.5 & 4 & 2 & 309.6 & 272 \\
\hline $\mathrm{MgBr}_{2}$ & 63.612 & 230.2 & 4 & 2 & 184.1 & 285 \\
\hline $\mathrm{NiBr}_{2}$ & 64.240 & 227.2 & 4 & 2 & 218.5 & 306 \\
\hline $\mathrm{NiI}_{2}$ & 65.453 & 224.1 & 4 & 2 & 312.5 & 334 \\
\hline
\end{tabular}

Based on the reversible reaction: Solid $\cdot(n+m) N_{3}+\Delta H \leftrightarrow$ Solid $\cdot n N_{3}+m H_{3}$ (gas)

A new class of microporous materials known as porous coordination polymers (PCPs) or metalorganic frameworks (MOFs) emerged recently [24]. These materials possess unique features such as huge surface area, large pore volume, and an unprecedented geometric, chemical, and physicochemical variability, which are due to their tunable composition. With regard to the application in adsorption heat transformation processes, the main focus lies on water adsorption properties and storage capacities, which are strongly connected to the specific pore volume. Several compounds with large water uptakes up to $1.43 \mathrm{~g} / \mathrm{g}$ have been identified. This is more 
than four times the maximum loading of conventionally used zeolites. With ongoing development, the specific pore volumes have increased from $1 \mathrm{~cm}^{3} / \mathrm{g}$ (MOF-5) up to more than $2 \mathrm{~cm}^{3} / \mathrm{g}$ for MIL-101 [322]. In combination with increasing pore volumes, extremely high surface areas up to $5000 \mathrm{~m}^{2} / \mathrm{g}$ have been reported for several MOFs. This has also started a discussion on the applied measurement methods, for example, the determination of the surface area by the BET method [323]. Another unique feature can be observed when dealing with the adsorption properties of MOFs. Some frameworks show geometric flexibility, that is, a reversible change in the structure and sometimes even in the physical properties in response to guest adsorption [324].
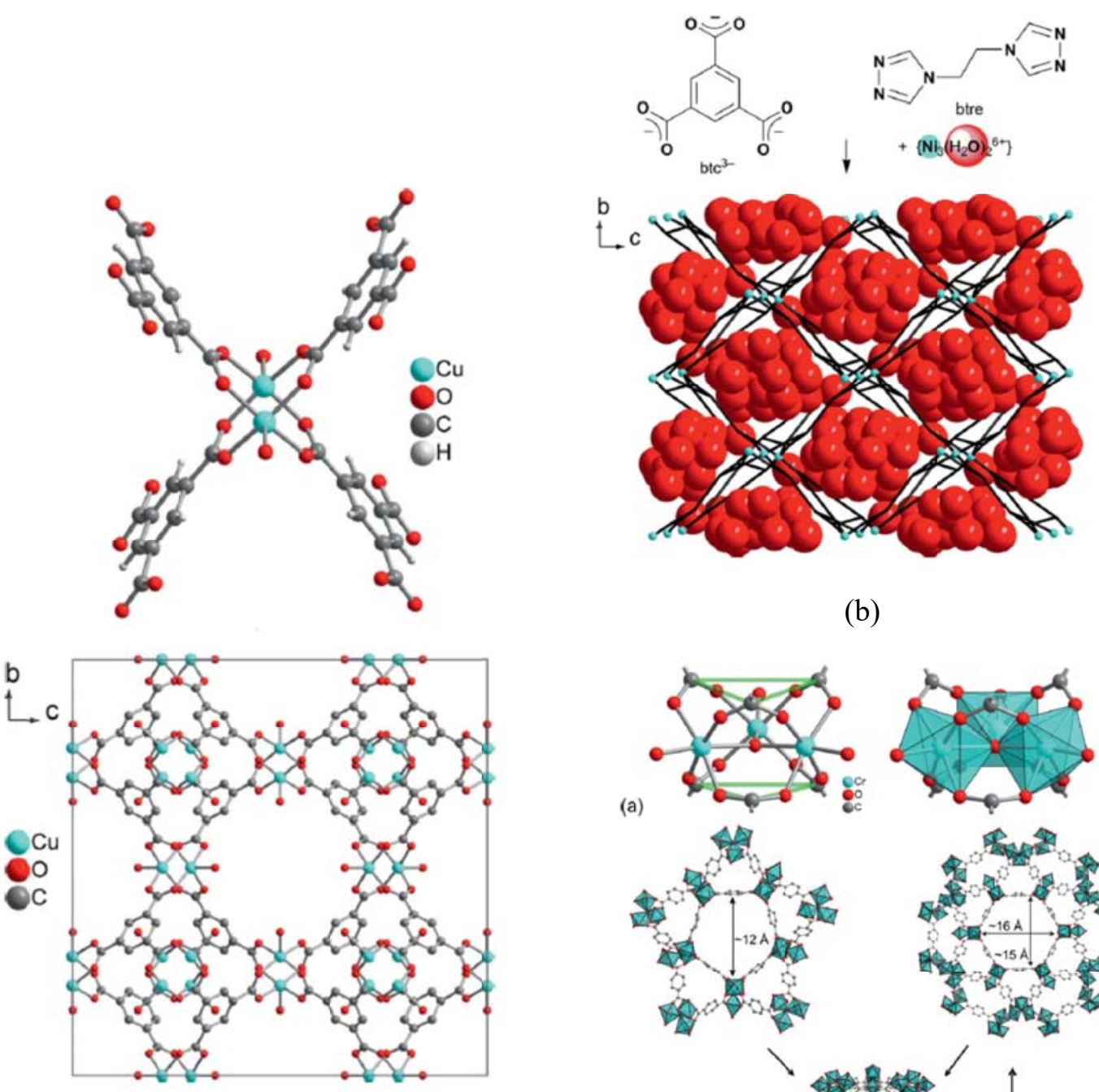

(b)
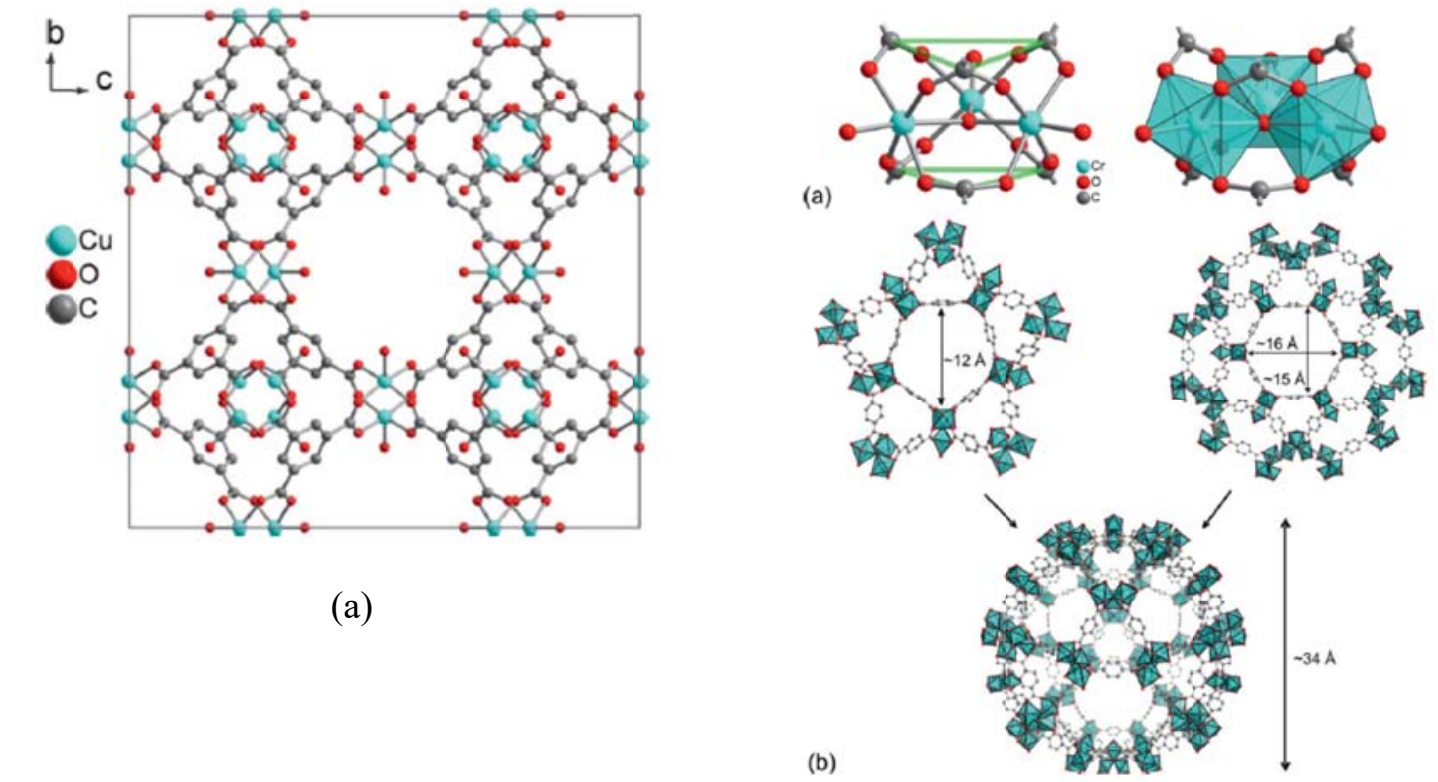

(c)

Figure 10. MOFs used in adsorption technologies [24]. (a) HKUST-1; (b) ISE-1; (c) MIL-101 
Compared to traditional adsorbents used in heat pump applications, like zeolites or aluminophosphates, MOFs exhibit a much richer variety in terms of composition, pore structure, and topology. Another advantage is the mechanism of synthesis. For applications in periodically working heat pumps or adsorption chillers, the comparatively weak thermal and especially hydrothermal stability of MOFs can be a disadvanges and is the most critical issue [325]. The most crucial point for hydrothermal stability is the nature of the metal-ligand coordinative bond.

Among different solid sorption refrigeration cycles, two operating strategies have been proposed and adopted for the improvement of system performance. In the first strategy, working performance can be improved by enlarging the cycled mass of refrigerant during adsorption phase using mass recover process between different sorption beds. In the second strategy, working performance can be improved by reducing the heat consumption during desorption phase using heat recovery process between different sorption beds. Later, the two strategies are combined to improve system performance by enlarging cooling capacity and reducing heat consumption simultaneously. These advanced sorption refrigeration cycles can significantly improve the thermodynamic efficiency and thus enhance the working performance. On the other hand, some advanced solid sorption refrigeration cycles have been proposed to lower the driving temperature to extend the reutilization of low-grade thermal energy. A summary of the working pairs used in the different strategies are presented in Table 9. In comparison with the basic solid sorption refrigeration cycle, advanced sorption cycles can improve the working performance significantly by introducing better heat management and mass recovery, or can effectively decrease the driving heat source temperature by employing two/multi-stage desorption sorption cycle. For advanced solid sorption refrigeration cycle with heat management strategy, heat recovery process is proposed to improve the working performance by reducing the heat consumption during desorption phase. For advanced solid sorption refrigeration cycle with mass recovery strategy, the working performance can be improved by enlarging the cycled mass of refrigerant when mass recovery process is introduced between different sorption beds. For advanced mass and heat recovery sorption refrigeration cycle, the aforementioned heat recovery and mass recovery strategies are combined to improve the working performance by enhancing cooling capacity and reducing heat consumption simultaneously. For advanced combined double-way thermochemical sorption cycle, both adsorption refrigeration and resorption refrigeration processes are combined to improve the cooling capacity. For advanced two/multi-stage sorption refrigeration cycle, the purpose is to lower the driving regeneration temperature in order to widen the scope of the reutilization of low-grade thermal energy instead of improving working performance. 


\section{Sorption thermal energy storage}

In the last decade, sorption storage has reached plenty of interest, especially because it is seen as high potential for long-term storage also known as seasonal storage. Nowadays, sorption storage technology and materials are still under research, but several prototypes are being tested bigger than lab scale. Different terminologies are found in the literature that might be confusing. Here, sorption storage technologies and materials are the focus of interest and comprise both chemical and physical adsorption and absorption as depicted in green in Figure 11.

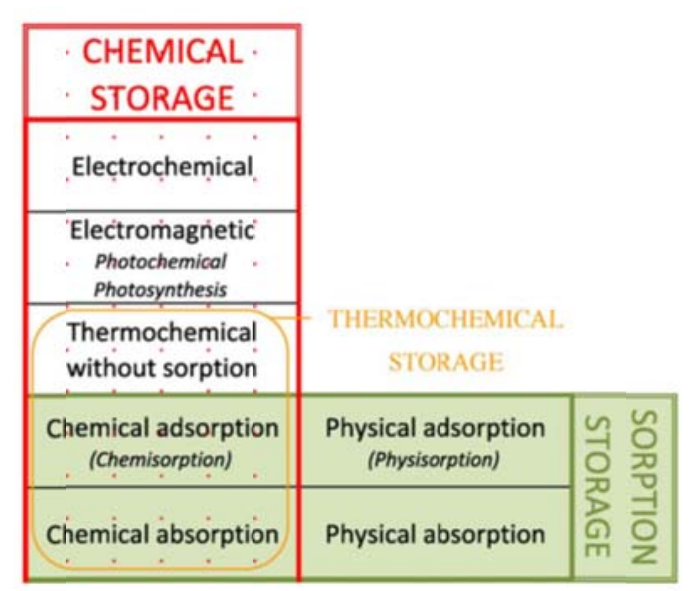

Figure 11. Sorption and chemical storage classification [327].

The concept of long-term energy storage or seasonal energy storage has become a research topic of interest in recent decades. Conventional storage methods can be sorted as short-term storage since heat input and output occur within an interval of several hours or days. Long-term energy storage is defined as storage systems designed to store energy for a few months or even a whole season to compensate for the seasonal offset of energy demand. In Table 11, the main characteristics with the aim of comparison of both short and long-term are shown for central solar heating systems. Sorption storage has been named as a potential technology for long-term solar energy storage, which is regarded as an efficient approach for making rational use of solar energy to achieve a considerable reduction of fossil fuel consumption and emission of $\mathrm{CO}_{2}$ $[6,328]$. 
Table 11. Main characteristics of central solar heating systems with short and long-term storage (adapted from $[6,328]$ )

\begin{tabular}{|l|l|l|}
\hline & Short-term storage & Long-term storage \\
\hline Storage time & Hours to days & 3 to 6 months \\
\hline Minimum size & More than 30 apartments & More than 100 apartments \\
\hline Yearly solar fraction & $10-20 \%$ & Over $50 \%$ \\
\hline Costs (Germany) & $0.08-0.15 € / \mathrm{kWh}$ & $0.17-0.40 € / \mathrm{kWh} / \mathrm{year}$ \\
\hline Flat plate collector (FPC) area & $0.8-1.2 \mathrm{~m}^{2} / \mathrm{person}$ & $\begin{array}{l}1.4-2.4 \mathrm{~m}^{2} / \mathrm{MWh} \text { annual } \\
\text { heat demand }\end{array}$ \\
\hline Storage volume & $0.05-0.1 \mathrm{~m}^{3}$ water $/ \mathrm{m}^{2} \mathrm{FPC}$ & $1.4-2.1 \mathrm{~m}^{3}$ water $/ \mathrm{m}^{2} \mathrm{FPC}$ \\
\hline
\end{tabular}

\subsection{Technologies}

Most of the technologies implementing sorption materials with storage are purposed for building comfort applications. Mainly air conditioning, heating, and DHW are the fulfilled requirements. Until now, these technologies have been designed tested as single equipment for a dwelling or as a buffer in district heating network.

\subsubsection{Adsorption}

Adsorption thermal energy storage is a promising technology that can provide an excellent solution for long-term thermal energy storage in a more compact and efficient way. Solar thermal energy or waste heat from several processes can be used to regenerate the adsorbent and promote the energy storage $[5,326]$.

Reactors designed for sorption thermal energy storage systems based on gas-solid processes (physisorption and chemisorption) can be either open or closed. Also, the reactor can act as the container of all solid (integrated) or have it as another equipment, named separated.

Open systems, as the name suggests, are open to the environment. The gas involved is typically water vapour. For the sorption process during discharging is obtained directly from the ambient air, as shown in Figure 12 left, or in some cases from a moisture source such as a humidifier. This makes open systems weather dependent. 


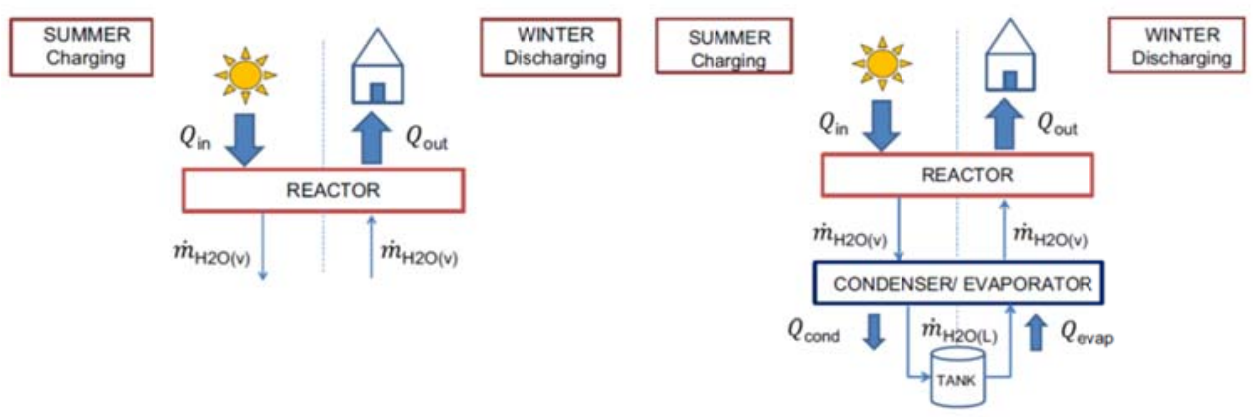

Figure 12. Open (left) vs. closed (right) systems with integrated reactor [329]

A closed adsorption system has no mass exchange with the environment and requires an evaporator/condenser for the working fluid, usually water, as can be seen in Figure 12 right. During the discharge process, evaporation of the working fluid is necessary and therefore a low heat source is required. Usually closed systems operate under vacuum conditions for optimal mass transport in the reactor [330]. When the working fluid is water the temperature of this heat source commonly needs to be at least at $5{ }^{\circ} \mathrm{C}$. It is not always mentioned how this heat should be supplied to the system, being still a key point that can make decide if as system is feasible or not [331].

On the contrary, the open system allows a simpler manufacturing of the reactor. The evaporator/condenser is not necessary, which does not affect negatively the apparent energy density of the storage system. Nevertheless, this working mode results in a flow of vapour and air through the bed, and the total flow is significantly higher than in the previous case. Thus, mass transfer must be carefully considered [332]. Moreover, although at the beginning of the reaction there is good vapour diffusion within these systems, as the time passes, the diffusion shows a decreasing trend and results in a corresponding drop in $\Delta \mathrm{T}$. This causes instability during heat generation, which is not evidenced in closed sorption systems. In closed cycles, the generated heat can be stored sensibly and the amount extracted can be altered by changing the parameters of the heat transfer fluids (e.g. mass flow rate) [333].

The closed sorption storage process is similar to that of chemical heat pumps with a major difference. A chemical heat pump works usually with two simultaneous circulations (continuous process) and needs therefore a condenser and an evaporator as well as a desorber and an ab/adsorber. Heat storage systems work in batch mode so that vessels are needed to store the products. In addition, the desorber might be used as an ab/adsorber and the condenser also as an evaporator [327]. 
A comparison between open and closed systems can be found in [327] and a numerical comparison has been published by Michel et al. [332], who concludes that for the closed system, the heat transfer is the main limitation, and a small increase of the thermal conductivity strongly improves the reaction rate. For example, a conductivity which is two times higher than the reference bed conductivity decreases the reaction time of about $14 \%$. For the open system, the mass transfer is the main limitation and the hydration rate can be improved by acting on the bed permeability: doubling the permeability leads to a half reaction time. Both operating modes lead to close global performances. Thus, the open thermochemical reactor, which presents technical advantages (easier conception and management, lower cost, etc.), is a promising way to implement a thermochemical process as long-term heat storage.

Other authors also compared open and closed systems but for physical adlsorption (zeolites 13X). The overall system energy and exergy efficiencies, respectively, are determined to be $50 \%$ and $9 \%$ for the closed storage, and $69 \%$ and $23 \%$ for the open storage. The results suggest that there is a significant margin for loss reduction and efficiency improvement for closed and open thermochemical storages, since the exergy efficiencies of both are significantly lower than the energy efficiencies [335].

Another possible configuration is to have an integrated or separate reactor. In the integrated reactor system (Figure 13b), the absorption/release of energy (reaction) occurs within the storage vessel, while the separate reactors (Figure 13a) concept consists in transporting the thermochemical material (TCM) from the storage vessel to the reactor and to another storage vessel, after reacting, as illustrated in Figure 13.


Figure 13. (a) Separated reactor, (b) reactor integrated in storage [336]

If the TCM material is a solid, preferably integrated reactors are used. This has the advantage that it is not necessary to transport the solid material, thus less power consumption. However, 
for large storages such as seasonal storage, this also has disadvantages such as a larger sensible heat loss, since then the whole reactor content has to be heated up before dehydration occurs [336]. Furthermore, the required heat exchange area is much larger than for a separate reactor. In a separate reactor, it is possible to heat up only the required amount of TCM, thereby making the charging process much more efficient. Also, the heat and vapour transfer can be optimised within a separate reactor by stirring of the material.

\section{a. Physisorption}

An open adsorption system was designed as a buffer for a district heating network in Munich by ZAE Bayern, as shown in Figure 14. The buffer can store $1300 \mathrm{kWh}$ for the heating during $14 \mathrm{~h}$ of a school building with a maximum power of $135 \mathrm{~kW}$. This system, which stores without loss as long as wanted, is charged in periods when energy is available from the net in off-peak hours and can therefore be independent from the net at peak demand periods. It contains zeolite $13 \mathrm{X}$ and offers a storage density of $124 \mathrm{kWh} / \mathrm{m}^{3}$ for heating and $100 \mathrm{kWh} / \mathrm{m}^{3}$ for cooling with a COP of 0.9 and 0.86 , respectively [327].
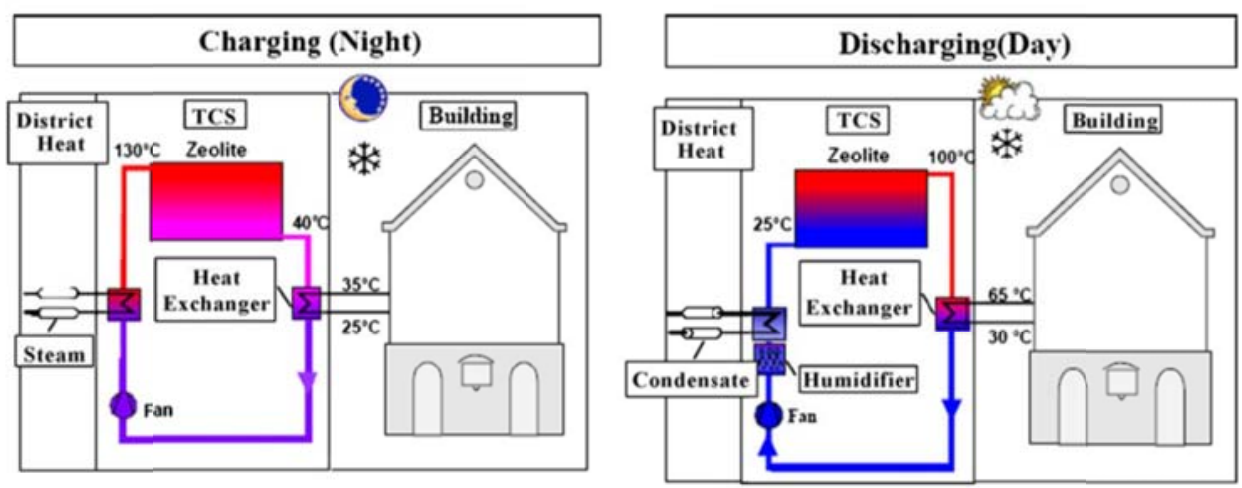

Figure 14. Open physisorption zeolites system on district heating net in Munich [337]

A prototype for heating and domestic hot water was built within HYDES project. The prototype consists on silica gel $/ \mathrm{H}_{2} \mathrm{O}$ as the sorption material combined with $20.4 \mathrm{~m}^{2}$ of solar collectors. The charging (desorption) temperature is given by the solar collectors at $88^{\circ} \mathrm{C}$. From the results it can be concluded that there is a $20 \%$ of losses regarding the storage density from the material to the system [327].

Another prototype based on silica gel $/ \mathrm{H}_{2} \mathrm{O}$, in the frame of Modestore project, was built consists of a store filled with the storage material and equipped with an internal spiral heat exchanger, a water reservoir (900 1) and an additional small water reservoir with a heat exchanger which 
serves as the evaporator or condenser. The tests of this prototype showed the unsuitability of the combination silica gel/ $\mathrm{H}_{2} \mathrm{O}$ for seasonal storage with flat-plate solar collectors $\left(32 \mathrm{~m}^{2}\right)$ and available heat sinks which do not allow desorption under water content of $3 \%$ when the material has to operate in a water content range of $3-13 \%$ [327,329]. This significantly reduces the storage density of the material. In the pilot system an energy storage density of approximately $50 \mathrm{kWh} / \mathrm{m}^{3}$ has been achieved. This is only $25 \%$ of the total energy storage density of the theoretical value $[327,329,338]$. A schematic drawing of the sorption store is depicted in Figure 15.

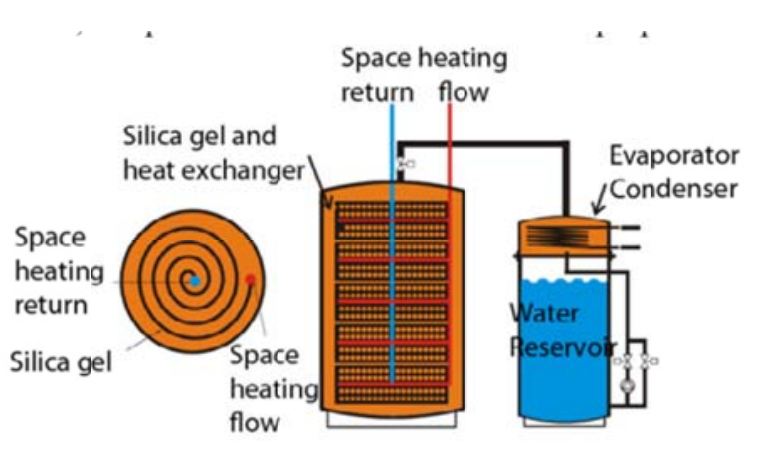

Figure 15 Modestore prototype based on silica gel/water as sorption material [329,338]

An open storage system containing zeolites was built in the frame of Monosorp project. Heat storage and removal from the store utilises the ventilation heat recovery system and moisture in the house $[338,339]$. The system was designed and tested showing promising results, however, the desorption temperature is very high, around $180{ }^{\circ} \mathrm{C}$, which makes the use of solar heat difficult with this concept [327].

In project SolSpaces prototype the aim is comparable to Monosorp and therefore the sorption system is integrated into an air heating system of a building which is equipped with a controlled ventilation system. The heat for desorption is provided by a collector field using air collectors. In this case the solar loop can be directly connected to the sorption store without the need for an air to water heat exchanger which was mandatory in the MonoSorp project. In contrast to the MonoSorp concept no zeolitic honeycomb monoliths are used as storage material but a packed bed of zeolite spheres.

Similar to the concepts of the MonoSorp and SolSpaces, CWS-NT-concept project is an open adsorption based on zeolites and salt as sorption material system using ambient or exhaust air to provide the humidity required for the discharging of the store. The thermochemical energy store works as a low power heat source and is connected to the combistore of the solar thermal system via the collector loop heat exchanger. The thermochemical energy store consists of a 
material reservoir for the storage material and a reactor where the heat and mass transfer take place during the charging and discharging reaction. In the CWS-NT-concept, an external reactor concept has been realized where the storage material is separated from the reactor. This has the advantage that the reaction is reduced to only a small part of the total storage material amount at a time [338].

\section{b. Chemisorption}

An open system (atmospheric) instead of the close system, being this choice based on a previous techno-economical study done by Zondag et al. [340], was built and tested. The sorption material is $\mathrm{MgCl}_{2} / \mathrm{H}_{2} \mathrm{O}$. The authors found that $\mathrm{HCl}$ was formed during the dehydration of $\mathrm{MgCl}_{2} \cdot 7 \mathrm{H}_{2} \mathrm{O}$ at a temperature above $135^{\circ} \mathrm{C}$. The formation of this gas not only degrades the storage material, but it is also strongly corrosive. Therefore, dehydration was limited to a temperature below $135{ }^{\circ} \mathrm{C}$. In addition, the over-hydration of $\mathrm{MgCl}_{2}$ was avoided by impregnation into a carrier material (cellulose). The measured temperature lift was $15^{\circ} \mathrm{C}$ and a significant temperature rise was maintained in the bed after 24 hours. The calculated energy density of the material after 23 hours hydration was $0.14 \mathrm{MJ} / \mathrm{kg}$ [341].

An open chemisorption storage system for long-term solar heat storage with magnesium chloride on a carrier matrix as storage material was tested. The prototype reactor contains 15 litters of storage material. The temperature for dehydrating the material is set to $130^{\circ} \mathrm{C}$. During the hydration experiments a temperature lift of the airflow of $14{ }^{\circ} \mathrm{C}$ from $50{ }^{\circ} \mathrm{C}$ to $64{ }^{\circ} \mathrm{C}$ has been measured in the material bed (relative humidity of the incoming airflow of $40 \%$ at $25^{\circ} \mathrm{C}$ ). With a further improvement of the reactor design and the heat transfer in the air to water and air to air heat exchanger it is expected that the high energy density of the material of approximately $280 \mathrm{kWh} / \mathrm{m}^{3}$ can be technically used [338].

Another project, with a prototype based on sodium sulphide as sorption material was built and tested. The project aimed to improve the performance of a sodium sulphide/water chemical energy storage previously designed [342]. Key aspects of this concept are the modular design (the reactor, the evaporator and the condenser aligned into the same and straight line), the integrated oil bath that results in a low temperature source required (about $79.5^{\circ} \mathrm{C}$ ) and the use of graphite additives. Graphite (30\% in weight) is mixed with the sodium sulfide nanohydrated salt $\left(\mathrm{Na}_{2} \mathrm{~S} \cdot 9 \mathrm{H}_{2} \mathrm{O}\right)$, reaching a COP of 1.11 (heating + cooling) [327]. 


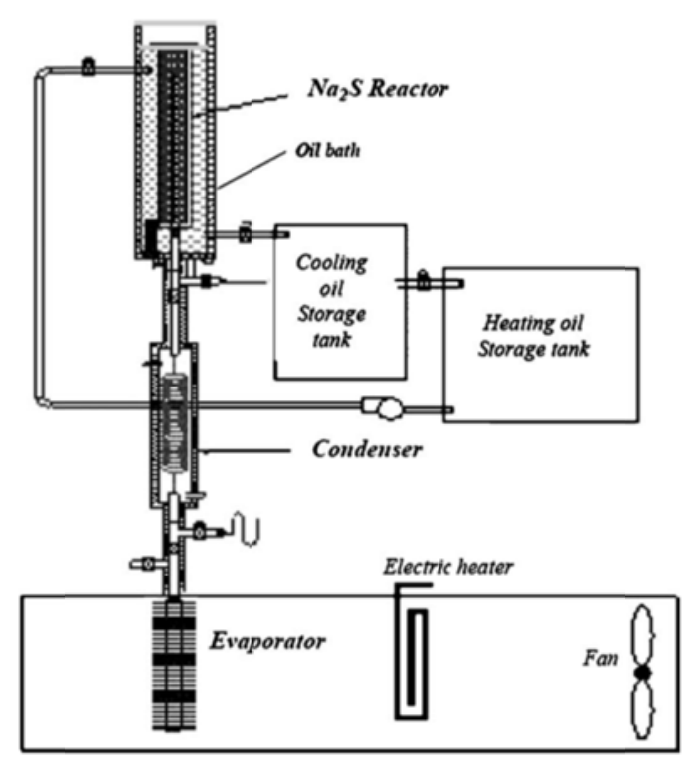

Figure 16. Scheme of the modular prototype based on $\mathrm{Na}_{2} \mathrm{~S} / \mathrm{H} 2 \mathrm{O}$ [343]

A prototype of modular energy storage system for cooling in buildings and industrial applications was developed and tested. The prototype is a closed spiro-tube heat exchanger containing sodium sulphide and water $\left(\mathrm{Na}_{2} \mathrm{~S} / \mathrm{H}_{2} \mathrm{O}\right)$ as the sorption material. Experimental results show a heat storage density of almost $1.1 \mathrm{kWh} / \mathrm{kg}$ and a cold storage of almost $0.7 \mathrm{kWh} / \mathrm{kg}$ [327]. The reported COP was 057 . Some solutions have been used to minimize corrosion: use of stainless steel for vessels and copper. Moreover, due to the melting point at $83{ }^{\circ} \mathrm{C}$ of the storage material, the upper limit for the condensation temperature was fixed at about $27{ }^{\circ} \mathrm{C}$. Nevertheless, the $\mathrm{Na}_{2} \mathrm{~S}$ was impregnated on fibrous cellulose to allow partial melting of the material without significant irreversibility. Peak cooling powers of 500 to $700 \mathrm{~W}$ were measured at evaporation temperature of 10 to $15^{\circ} \mathrm{C}$, which remained relatively constant until all the water was evaporated. The measured maximum thermal storage capacities of the prototype were $3.2 \mathrm{kWh}$ for heat and $2.1 \mathrm{kWh}$ for cold [341].

A solar assisted sorption system using $\mathrm{SrBr}_{2}$ was tested [344]. The system was designed for heating floor in winter or mid-season at $35{ }^{\circ} \mathrm{C}$ and refreshing floor in summer at $18{ }^{\circ} \mathrm{C} . \mathrm{SrBr}_{2}$ was mixed with expanded graphite and compacted. The reported mean cooling power was 48 $\mathrm{kW} \cdot \mathrm{m}^{-3}$ at the operating temperature of $80^{\circ} \mathrm{C} / 35^{\circ} \mathrm{C} / 18^{\circ} \mathrm{C}$ (summer) whereas the heating power in winter was $36 \mathrm{~kW} \cdot \mathrm{m}-3$ at the operating temperature of $70{ }^{\circ} \mathrm{C} / 35^{\circ} \mathrm{C} / 12{ }^{\circ} \mathrm{C}$ (winter). The average powers were calculated from the experimental measurements obtained between 0 to 90 $\%$ of conversion. A prototype reactor that was able to store, with a complete reaction, $60 \mathrm{kWh}$ and $40 \mathrm{kWh}$ for heating and cooling respectively was built. In summer conditions $\left(66^{\circ} \mathrm{C} / 35-30\right.$ $\left.{ }^{\circ} \mathrm{C} / 18^{\circ} \mathrm{C}\right)$, the mean refreshing power was $-2.6 \mathrm{~kW}$ during the $14 \mathrm{~h}$ of the sorption period $(0-92$ 
$\%$ ). On the other hand, in mid-summer conditions $\left(70{ }^{\circ} \mathrm{C} / 12-30{ }^{\circ} \mathrm{C} / 12{ }^{\circ} \mathrm{C}\right)$, the mean heating power was $2.2 \mathrm{~kW}$ during the first half of the sorption process (55\% conversion) [341].

A demonstration plant of the MERITS project containing numerous subsystems such as solar collectors, short term storage vessel, long term thermochemical storage modules (based on $\mathrm{Na}_{2} \mathrm{~S}$ ), underfloor heating, ceiling cooling, and a domestic hot water system, was designed during the first two years of the project (2012-2014). All compartments, i.e. the heating \& cooling systems, the technical space and the single family dwelling compartment complete with windows, ventilation and door, are integrated in a $45 \mathrm{ft}$. sea container and fully equipped with measuring equipment (Figure 17). The long term thermochemical storage system (heat battery) consists of 8 modules providing a loss-free design thermal energy storage capacity of $482 \mathrm{MJ}$ delivered from the water and sodium sulphide $\left(\mathrm{Na}_{2} \mathrm{~S}\right)$ working-pair. As described in formula (1), the $\mathrm{Na}_{2} \mathrm{~S}$ is supposed to be cycling between the hemihydrate $\left(\mathrm{Na}_{2} \mathrm{~S} \cdot 1 / 2 \mathrm{H}_{2} \mathrm{O}\right)$ and the pentahydrate $\left(\mathrm{Na}_{2} \mathrm{~S} \cdot 5 \mathrm{H}_{2} \mathrm{O}\right)$. The ground source, simulated using a laboratory water bath, is used for evaporation and condensation. With an evaporator inlet temperature of $10^{\circ} \mathrm{C}$, the TCS modules can provide a temperature of up to $60{ }^{\circ} \mathrm{C}$ to the short term buffer vessel, to anticipate the thermal demands during the winter period, with a constant power. After pre-testing the 8 thermochemical storage modules were introduced for winter testing in Warsaw, Poland. In a first test with a not yet optimized discharging control strategy an energy amount of 13.5 MJ could be discharged from one module [345,347].

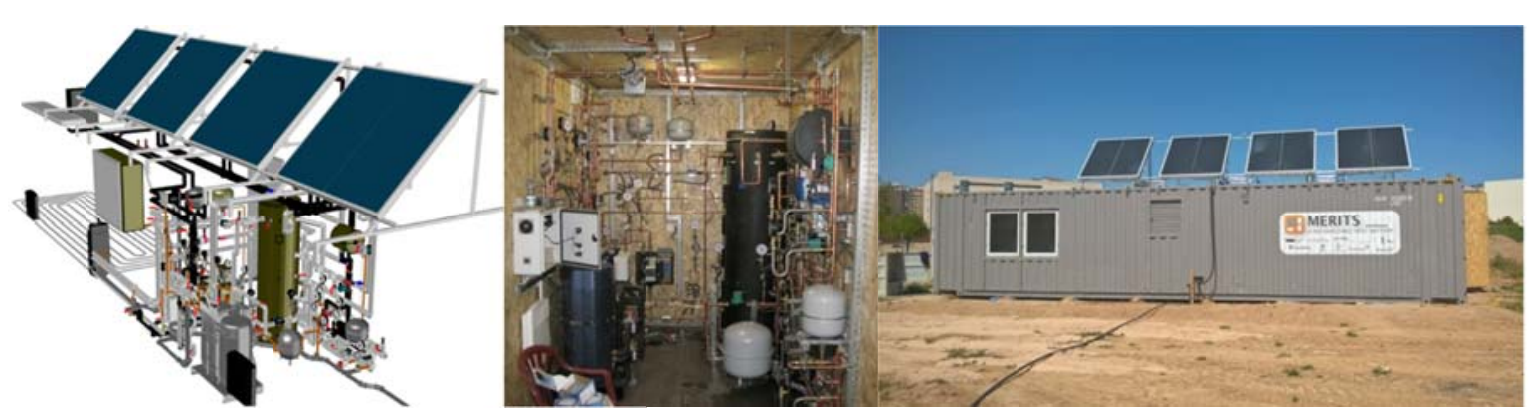

Figure 17. Field Test Demonstrator 3D systems model (left), interior view of the energy processing compartment (middle), and Field Test Demonstration container during pre-testing in Lleida, Spain (right)

A novel thermochemical sorption cycle is proposed to upgrade the stored thermal energy by using a pressure-reducing desorption method during energy storage process and a temperaturelift adsorption technique during energy release process (Figure 18). Theoretical analysis showed that the proposed target-oriented thermochemical sorption heat transformer is effective for the integrated energy storage and energy upgrade, and the low-grade thermal energy can be upgraded from $87{ }^{\circ} \mathrm{C}$ to $171{ }^{\circ} \mathrm{C}$ using a group of sorption working pair $\mathrm{MnCl}_{2}-\mathrm{CaCl}_{2}-\mathrm{NH}_{3}$. 
Moreover, it can give the flexibility of deciding the temperature magnitude of energy upgrade by choosing appropriate sorption working pairs [246,348].

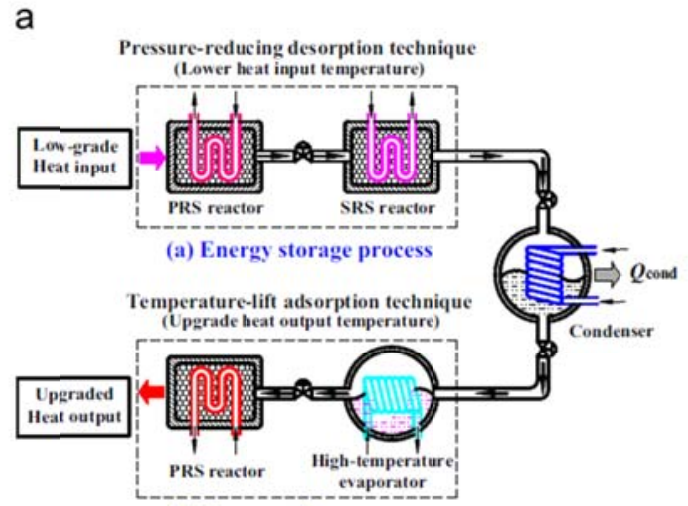

(b) Energy release process



Figure 18. Schematic diagram of the novel target-oriented solid-gas chemical sorption heat transformer for the integrated energy storage and energy upgrade [348]. (a) Diagram of the physical arrangement of system components of the novel target-oriented solid-gas chemical sorption heat transformer and (b) Diagram of Clausius-Clapeyron

In the same direction, a dual-mode solid gas thermochemical sorption system is proposed for seasonal storage of solar thermal energy with little heat losses during long-term storage (Figure 19). In this case, the proposed system has two working modes which operate according to the different ambient temperature in winter. Defining the reactor mass ratio ( $\left.\mathrm{R}^{\mathrm{i}}\right)$, as the mass of metallic parts of reactor heat exchanger (including vessels, tubes, fins, and so on) divided by the mass of the reactive salt in a solid-gas reactor, is employed to assess the effect of the thermal capacity of the metallic part of reactor on the COP for heat production $\left(\mathrm{COP}_{h}\right)$ and energy density of the proposed thermochemical sorption energy storage system.

At mass ratio of 5 , global conversion of 0.85 , and heat output temperature of $62{ }^{\circ} \mathrm{C}$, the $\mathrm{COP}_{\mathrm{h}}$ and energy density obtained from the thermochemical seasonal energy storage process without internal heat recovery are 0.60 and $1043 \mathrm{~kJ} / \mathrm{kg}_{\text {salt }}$ when the ambient temperature is $0{ }^{\circ} \mathrm{C}$ in winter; these figures are 0.34 and $579 \mathrm{~kJ} / \mathrm{kg}_{\text {salt }}$, respectively, for the thermochemical seasonal energy storage process with internal heat recovery at an ambient temperature of $20^{\circ} \mathrm{C}[348]$. 
a

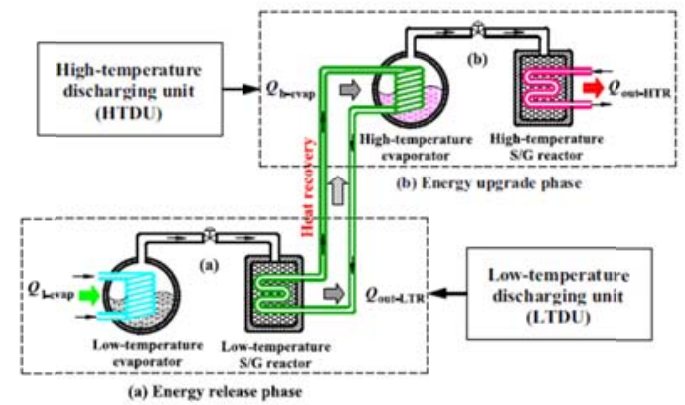

b

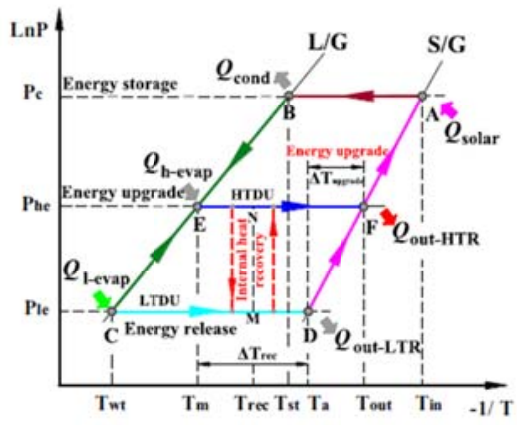

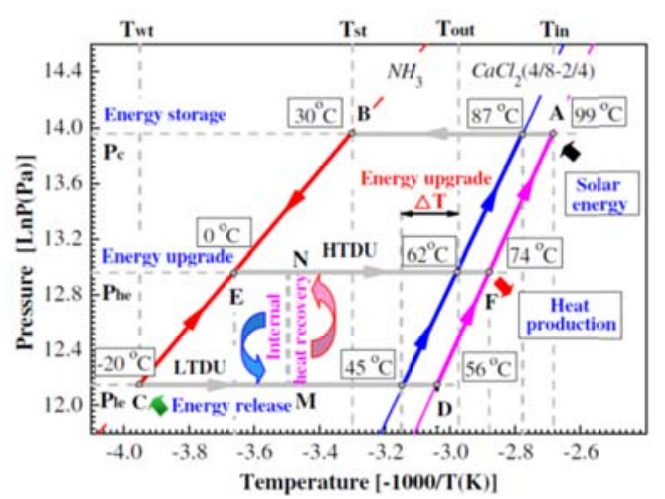

Figure 19. Schematic diagram of the dual-mode solid-gas chemical sorption system for seasonal storage of solar thermal energy [349]. (a)Internal heat recovery process between reactor in LTDU and evaporator in HTDU and Energy storage process: A-B; Energy upgrade process of HTDU: E-F; Energy release process of LTDU:C-D; Internal heat recovery process: M-N (b) Clausius-Clapeyron diagram (c) Clapeyron diagram of the integrated energy storage and energy upgrade thermochemical sorption system using sorption working pair $\mathrm{CaCl}_{2}-\mathrm{NH}_{3}$.

\subsubsection{Absorption}

As mentioned in adsorption section, those systems can be classified as open or closed. In absorption processes, only closed systems are found and are comparable with closed adsorption systems in the sense of presenting two essential components being the condenser and the evaporator. In the case of the reactor the main differences appear. While a generator and absorber are in absorption technologies, an adsorber and desorber are the core of the adsorption technologies. This is exemplified in Figure 20. 

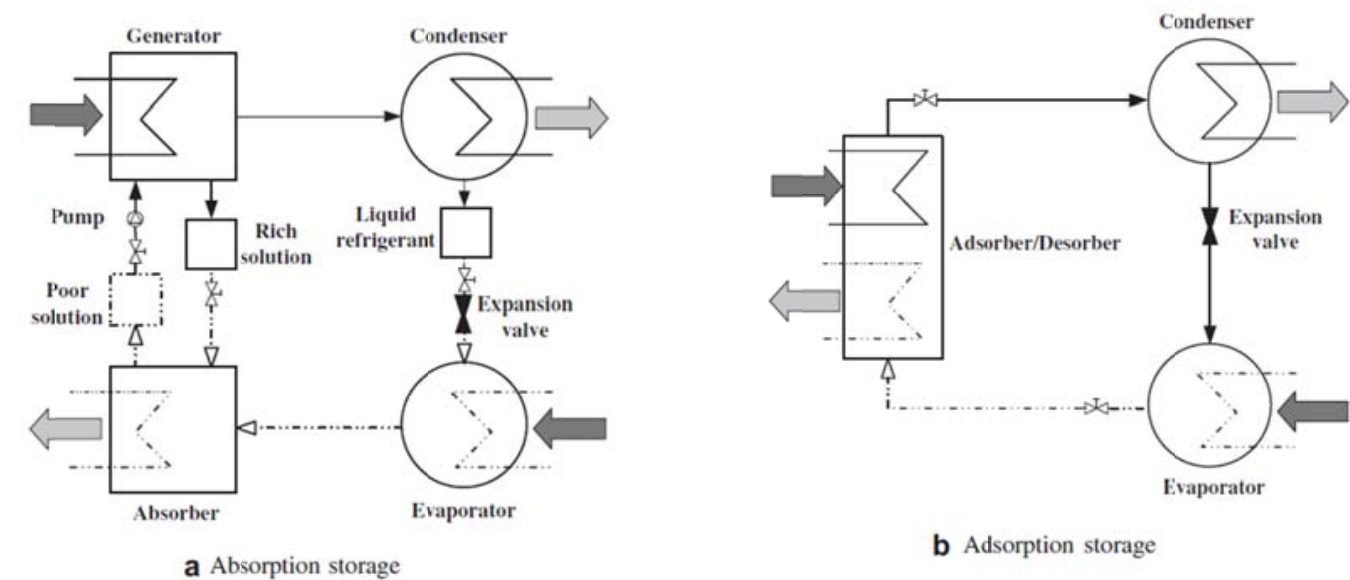

Figure 20. Comparison schemes between (a) absorption and (b) adsorption closed systems [3]

Moreover, and as shown in Figure 21, within the closed absorption technologies, single-stage or double-stage have been designed for sodium hydroxide and water, $\mathrm{NaOH} / \mathrm{H}_{2} \mathrm{O}$, as absorption pair [327,350]. In single-stage, during charging phase, solar heat is supplied to the lowconcentration solution in the regenerator. The water vapour is desorbed, flows to the condenser where it condenses. The condensated water is collected inside the water storage tank. To discharge the $\mathrm{NaOH}$ storage, the stored water is evaporated in an evaporator using a low temperature source. The vapour is absorbed by the highly concentrated $\mathrm{NaOH}$ lye in the absorber and heat is released. The main advantage of $\mathrm{NaOH}$ is its cost in relation with its storage density. The energy storage density depends on the charging and discharging temperature and is between 170 and $400 \mathrm{kWh} / \mathrm{m}^{3}$. The process has been successfully demonstrated in a prototype plant with a capacity of 2001 of sodium hydroxide [338].

A double-stage system (Figure 21 right) was designed to improve the heat production temperature level during discharging process or to reduce the required temperature level during charging process. The overall working principle is similar to the common system (single-stage process) except for an added regenerator 2, condenser 2 and heat exchanger. To regenerate the storage, the $\mathrm{NaOH}$ solution flows from regenerator 1 and is split up into two flows: the first part enters condenser 2 and the second part enters regenerator 2. The condenser 2 is working as an absorber, which absorbs the vapour desorbed in regenerator 2. The intermediate concentration solution that leaves condenser 2 returns to regenerator 1 . As the pressure in regenerator 2 is almost the same in condenser 2, which is lower than that in regenerator 1 , the concentration of the solution can be increased at lower temperature level $\left(95^{\circ} \mathrm{C}\right)$ than that required in regenerator 1 to reach the same concentration $\left(150^{\circ} \mathrm{C}\right)$. The main drawbacks of double-stage processes are the decrease in storage density and the complexity of the system, which needs more tanks and heat exchangers [327]. 

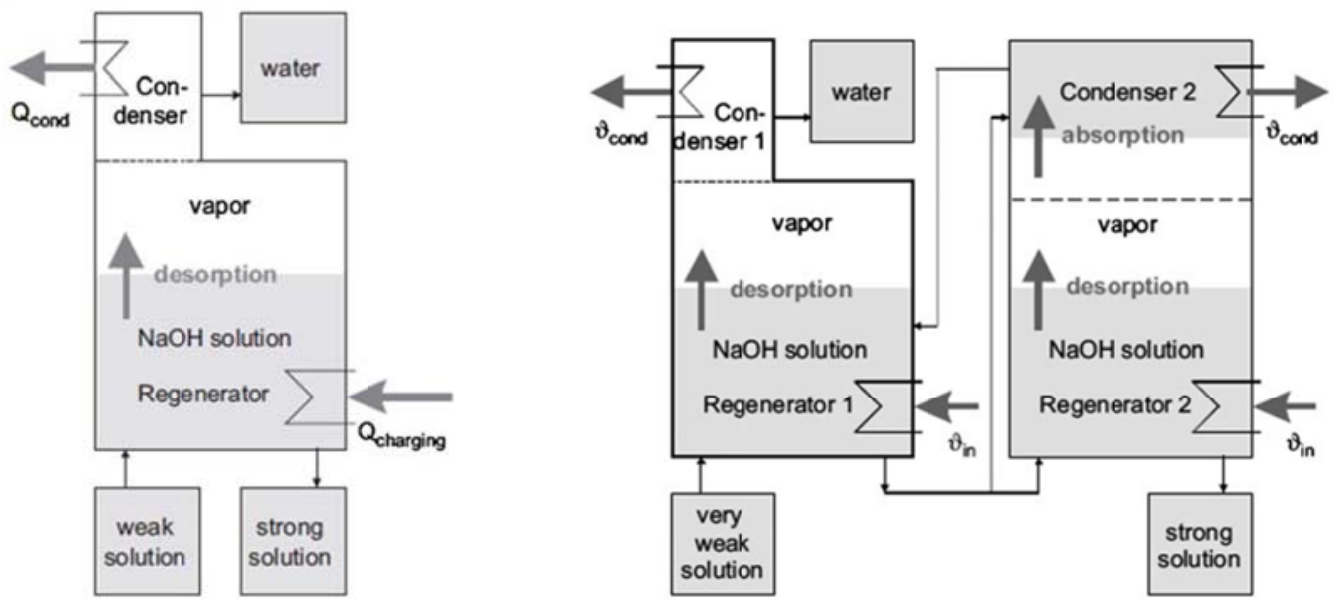

Figure 21. Operating principle (charging) of closed absorption storage technology, left: one stage, right: double-stage [350].

The system based on lithium chloride, $\mathrm{LiCl}$, can deliver either cooling (cooling mode) or heating (heating mode). Crystallization, a long time bottleneck point, has been overcome and that enables the enhancement of the storage density and the COP with a so-called "closed three phase absorption" ( $\mathrm{LiCl}$ solution, salt crystals and water) [351]. The storage density experimentally reached is $253 \mathrm{kWh} / \mathrm{m}^{3}$ of $\mathrm{LiCl}$ salt. However, the high price of $\mathrm{LiCl}$ led the authors to dismiss prospect of long-term storage, as said before. For charging, poor solution is pumped over the heat exchanger and comes closer and closer to the saturation point so that solid crystals are formed and collected in the vessel, as they fell under gravity. A sieve prevents them from following the solution into the pump. The water vapour, which is desorbed, flows into the condenser by the gas pipe. The process is reversed during discharging, where the solution becomes unsaturated on the heat exchanger by absorbing the water vapour produced in the condenser (which becomes the evaporator). The poor solution falls into the vessel where it becomes saturated because of the dissolution of some crystals. The heat of evaporation is provided either by the building (cooling mode) or by the environment (heating mode). The heat of condensation and binding energy release is transferred to the environment (cooling mode) or to the building (heating mode) [327]. 


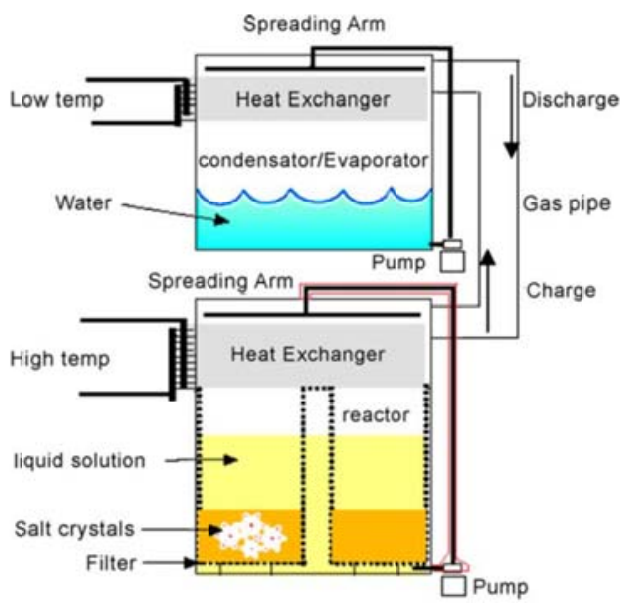

Figure 22. Absorption system based on $\mathrm{LiCl}$ [351]

Aqueous calcium chloride was investigated as long-term solar storage medium. The absorption system consist on a closed concept with just one vessel for both, concentrated and diluted solution of aqueous calcium chloride $\left(\mathrm{CaCl}_{2}\right)$. The closed storage concept combines absorption and sensible heat storage and achieves an energy density of approximately $105 \mathrm{kWh} / \mathrm{m}^{3}$ [352]. An experimental prototype with a 15001 store has been set up and it could be demonstrated that the mixing rate of concentrated and diluted solution is very small [338].

Another absorption setup was designed on the basis of sulphuric acid and water [353]. In Figure 23 the scheme of the system can be observed. Two liquids were mixed in two different systems of multistage mixing column, and an application of heat of dilution to multipurpose energy utilization was analysed. The utility of heat of dilution is explained from the results of thermal characteristics of each system [353].

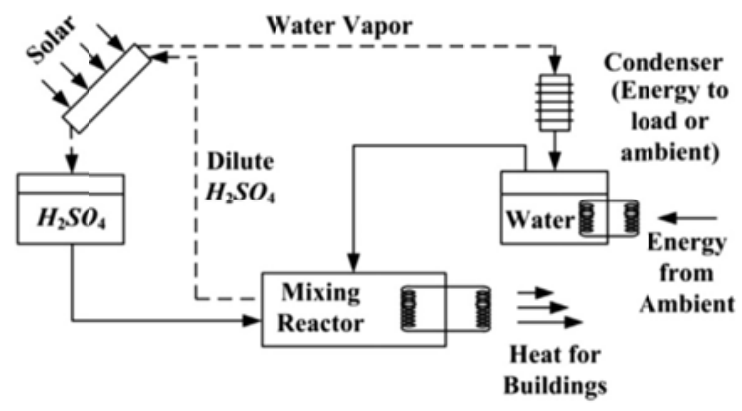

Figure 23. Absorption heat storage for sulphuric acid concentration difference [348,354] 
Table 12. Sorption technologies along with its application and main features

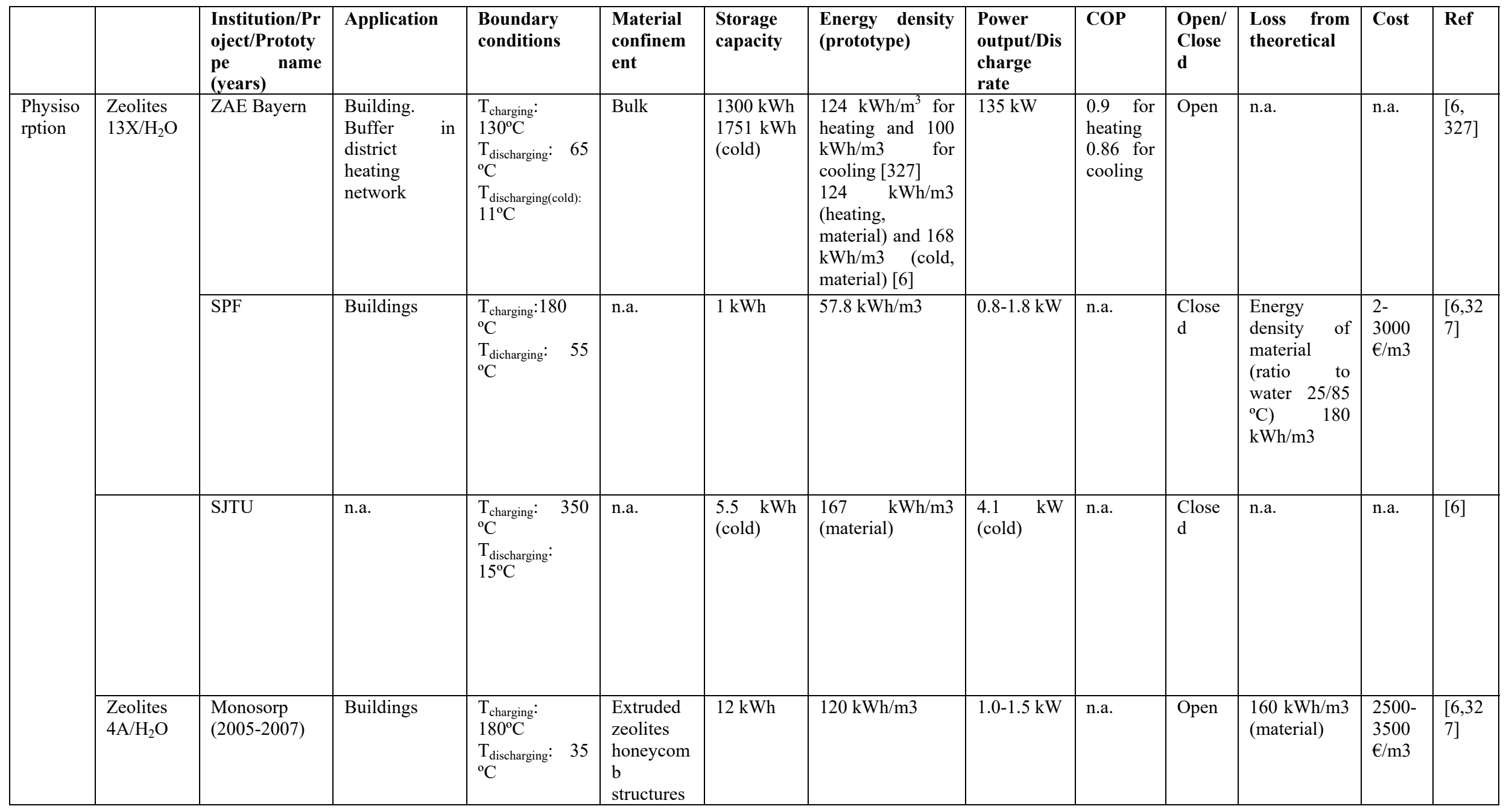




\begin{tabular}{|c|c|c|c|c|c|c|c|c|c|c|c|c|c|}
\hline & $\begin{array}{l}\text { Silica } \\
\text { gel } / \mathrm{H}_{2} \mathrm{O}\end{array}$ & Hydes & $\begin{array}{l}\text { Building. } \\
\text { Heating and } \\
\text { DHW }\end{array}$ & $\mathrm{T}_{\text {charging }}: 88^{\circ} \mathrm{C}$ & n.a. & n.a. & $\begin{array}{l}120 \mathrm{kWh} / \mathrm{m} 3 \text { of } \\
\text { silica gel }\end{array}$ & n.a. & n.a. & $\begin{array}{l}\text { Close } \\
\mathrm{d}\end{array}$ & $20 \%$ & n.a. & {$[327]$} \\
\hline & & Modestore & $\begin{array}{l}\text { Building. } \\
\text { Heating } \\
\text { single-family } \\
\text { house }\end{array}$ & $\begin{array}{l}\mathrm{T}_{\text {discharging: }}: 88 \\
{ }^{\circ} \mathrm{C}\end{array}$ & n.a. & $13 \mathrm{kWh}$ & $33.3 \mathrm{kWh} / \mathrm{m} 3$ & $\begin{array}{ll}0.5- & 1.0 \\
\mathrm{~kW} & \end{array}$ & n.a. & $\begin{array}{l}\text { Close } \\
\mathrm{d}\end{array}$ & $\begin{array}{lr}\begin{array}{l}\text { Energy } \\
\text { density } \\
\text { material }\end{array} \\
\text { (ratio } \\
\text { water } & \text { to } \\
{ }^{\circ} \mathrm{C} \text { ) } & 50 \\
\mathrm{kWh} / \mathrm{m} 3 & \end{array}$ & $\begin{array}{l}4300 \\
€ / \mathrm{m} 3\end{array}$ & $\begin{array}{l}{[6,32} \\
7]\end{array}$ \\
\hline \multirow[t]{6}{*}{$\begin{array}{l}\text { Chemis } \\
\text { orption }\end{array}$} & $\begin{array}{l}\mathrm{MgCl}_{2} / \mathrm{H}_{2} \\
\mathrm{O}\end{array}$ & ECN (2013) & Heating & $\begin{array}{l}\mathrm{T}_{\text {discharging: }}: 64 \\
{ }^{\circ} \mathrm{C}\end{array}$ & bulk & $2.4 \mathrm{kWh}$ & $\begin{array}{l}139 \quad \mathrm{kWh} / \mathrm{m} 3 \\
\text { (material) [6] } \\
0.5 \mathrm{GJ} / \mathrm{m} 3[329]\end{array}$ & $0.15 \mathrm{~kW}$ & n.a. & $\begin{array}{l}\text { Close } \\
\mathrm{d}\end{array}$ & n.a. & n.a. & $\begin{array}{l}{[6,32} \\
9]\end{array}$ \\
\hline & $\begin{array}{l}\mathrm{MgSO}_{4} \cdot 7 \\
\mathrm{H}_{2} \mathrm{O}\end{array}$ & ECN & Buildings & $150^{\circ} \mathrm{C}$ & n.a. & n.a. & $420 \mathrm{kWh} / \mathrm{m} 3$ & n.a. & n.a. & $\begin{array}{l}\text { Close } \\
\mathrm{d}\end{array}$ & n.a. & $\begin{array}{l}4870 \\
€ / \mathrm{m} 3\end{array}$ & {$[327]$} \\
\hline & $\begin{array}{l}\mathrm{Na}_{2} \mathrm{~S} / \mathrm{H}_{2} \mathrm{O} \\
+ \\
\text { cellulose }\end{array}$ & ECN & n.a. & $\begin{array}{l}\mathrm{T}_{\text {discharging: }}: 5- \\
20^{\circ} \mathrm{C}\end{array}$ & n.a. & $\begin{array}{l}3.2 \mathrm{kWh} \\
2.1 \mathrm{kWh} \\
\text { (cold) }\end{array}$ & $\begin{array}{ll}1070 & \mathrm{Wh} / \mathrm{kg} \\
\text { (material) } \\
700 & \mathrm{Wh} / \mathrm{kg} \\
\text { (material, cold) }\end{array}$ & $0.5-0.7 \mathrm{~kW}$ & n.a. & $\begin{array}{l}\text { Close } \\
\mathrm{d}\end{array}$ & n.a. & n.a. & [6] \\
\hline & $\begin{array}{l}\mathrm{Na} 2 \mathrm{~S}+\text { gra } \\
\text { phite/H2O }\end{array}$ & $\begin{array}{l}\text { Chiang Ma } \\
\text { University }\end{array}$ & Buildings & $\begin{array}{l}\mathrm{T}_{\text {charging: }}: 80-95 \\
{ }^{\circ} \mathrm{C}\end{array}$ & n.a. & $0.56 \mathrm{kWh}$ & $\begin{array}{ll}2240 & \mathrm{Wh} / \mathrm{g} \\
\text { (material) } & \end{array}$ & $\begin{array}{l}0.15-0.24 \\
\mathrm{~kW}\end{array}$ & n.a. & $\begin{array}{l}\text { Close } \\
\text { d }\end{array}$ & n.a. & n.a. & {$[6]$} \\
\hline & $\begin{array}{l}\text { SrBr2+Ex } \\
\text { panded } \\
\text { graphite/H } \\
2 \mathrm{O}\end{array}$ & $\begin{array}{l}\text { PROMES } \\
2008\end{array}$ & $\begin{array}{l}\text { Heating and } \\
\text { cooling }\end{array}$ & $\begin{array}{l}\mathrm{T} \text { charging: } 80 \\
{ }^{\circ} \mathrm{C}\end{array}$ & n.a. & $\begin{array}{l}60 \mathrm{kWh} \\
\text { for } \\
\text { heating } \\
40 \mathrm{kWh} \\
\text { for } \\
\text { cooling }\end{array}$ & n.a. & $2.5-4.0 \mathrm{~kW}$ & n.a. & $\begin{array}{l}\text { Close } \\
\mathrm{d}\end{array}$ & n.a. & n.a. & $\begin{array}{l}{[6,32} \\
9]\end{array}$ \\
\hline & $\begin{array}{l}\mathrm{BaCl} 2+ \\
\text { expanded } \\
\text { graphite/a } \\
\text { mmonia }\end{array}$ & $\begin{array}{l}\text { PROMES } \\
2012\end{array}$ & $\begin{array}{l}\text { Solar air } \\
\text { conditioning }\end{array}$ & $\begin{array}{l}\mathrm{T}_{\text {charging }}: 60-70 \\
{ }^{\circ} \mathrm{C} \\
\mathrm{T}_{\text {discharging }}: \\
{ }^{\circ} \mathrm{C}\end{array}$ & tubes & $\begin{array}{l}20 \mathrm{kWh} \\
\text { (cold) }\end{array}$ & $114 \mathrm{Wh} / \mathrm{kg}$ (cold) & $\begin{array}{ll}5 & \mathrm{~kW} \\
\text { (cold) } & \end{array}$ & n.a. & $\begin{array}{l}\text { Close } \\
\mathrm{d}\end{array}$ & n.a. & n.a. & $\begin{array}{l}{[6,32} \\
9]\end{array}$ \\
\hline
\end{tabular}




\begin{tabular}{|c|c|c|c|c|c|c|c|c|c|c|c|c|c|}
\hline \multirow[t]{3}{*}{$\begin{array}{l}\text { Physiso } \\
\text { prtion+ } \\
\text { chemiso } \\
\text { rption }\end{array}$} & $\begin{array}{l}\text { Zeolites } \\
13 \mathrm{X} \\
15 \% \\
\mathrm{MgSO}_{4} / \mathrm{H} \\
{ }_{2} \mathrm{O}\end{array}$ & $\begin{array}{l}\text { SolsSpaces } \\
(2012-2014)\end{array}$ & n.a. & n.a. & $\begin{array}{l}\text { packed } \\
\text { bed of } \\
\text { zeolite } \\
\text { spheres }\end{array}$ & n.a. & n.a. & n.a. & n.a. & n.a. & n.a. & n.a. & {$[338]$} \\
\hline & $\begin{array}{ll} & \text { Z } \\
\text { eolites } & + \\
\text { salt } & \end{array}$ & $\begin{array}{l}\text { CWS-NT- } \\
\text { concept }\end{array}$ & n.a. & $\begin{array}{l}\mathrm{T}_{\text {charging: }}: 130 \\
{ }^{\circ} \mathrm{C}\end{array}$ & n.a. & n.a. & n.a. & n.a. & n.a. & n.a. & n.a. & n.a. & [6] \\
\hline & $\begin{array}{l}\text { Attalpugit } \\
\text { e }+30 \% \\
\mathrm{CaCl}_{2} \\
/ \mathrm{H}_{2} \mathrm{O}\end{array}$ & $\begin{array}{l}\text { Fraunhofer } \\
\text { IGb, SeoSys } \\
\text { GmbH } 2012\end{array}$ & Heating & n.a. & n.a. & $\begin{array}{l}200 \\
\mathrm{Wh} / \mathrm{kg}\end{array}$ & n.a. & n.a. & n.a. & Open & $\begin{array}{l}\text { Scaling } \\
\text { effects } \\
\text { observed }\end{array}$ & n.a. & [329] \\
\hline \multirow[t]{3}{*}{$\begin{array}{l}\text { Absorpt } \\
\text { ion }\end{array}$} & $\begin{array}{l}\mathrm{NaOH} \\
\text { Two } \\
\text { phase }\end{array}$ & EMPA & Buildings & $\begin{array}{l}\mathrm{T}_{\text {charging }}: 93^{\circ} \mathrm{C} \\
\mathrm{T}_{\text {discharging }}: \quad 70 \\
{ }^{\circ} \mathrm{C}\end{array}$ & n.a. & $8.9 \mathrm{kWh}$ & $5 \mathrm{kWh} / \mathrm{m} 3$ & $1 \mathrm{~kW}$ & n.a. & $\begin{array}{l}\text { Close } \\
\mathrm{d}\end{array}$ & $\begin{array}{l}\begin{array}{l}\text { Energy } \\
\text { density } \\
\text { material of } \\
\text { (ratio } \\
\text { water }\end{array} \text { to } \\
{ }^{\circ} \mathrm{C} \text { ) } \\
\mathrm{kWh} / \mathrm{m} 3\end{array}$ & $\begin{array}{l}250 \\
€ / \mathrm{m} 3\end{array}$ & $\begin{array}{l}{[6,32} \\
7]\end{array}$ \\
\hline & \multirow[t]{2}{*}{$\mathrm{LiBr} / \mathrm{H} 2 \mathrm{O}$} & n.a. & $\begin{array}{l}\text { Air } \\
\text { conditioning }\end{array}$ & n.a. & $\begin{array}{l}\text { Dilute } \\
\text { solution } \\
\text { volume, } \\
\text { concentrat } \\
\text { ed } \\
\text { solution } \\
\text { and water } \\
\text { volume } \\
\end{array}$ & 58.2 & n.a. & n.a. & n.a. & n.a. & n.a. & n.a. & [3] \\
\hline & & n.a. & $\begin{array}{l}\text { Air } \\
\text { conditioning }\end{array}$ & n.a. & $\begin{array}{l}\text { Mixed } \\
\text { storage } \\
\text { volume } \\
\text { and water } \\
\text { volume }\end{array}$ & 116.7 & n.a. & n.a. & n.a. & n.a. & n.a. & n.a. & [3] \\
\hline
\end{tabular}









\subsection{Materials}

Sorption storage materials are usually classified as the technologies, absorption and adsorption, where one can find physisorption and chemisorption. Then, mixtures between these last two, physisorption and chemisorption, are being developed and tested and thus are referred as composites. Composites can also include mixtures of any sorption material with another inert material such as graphite, cellulose, etc. In Figure 24 a possible classification is given, where sorption materials are divided in four groups. Chemical reactions group can also be known as chemisorption materials and more in a wider concept as thermochemical materials. Thermochemical materials also encompass chemical reactions without sorption, such as redox reactions for high temperature thermal energy storage [355], which is out of the scope of the present review. Some of the sorption storage materials are also implemented in sorption technologies without storage, under different conditions. More fundamental information regarding sorption materials and its properties in a non-storage technology can be found in section 2.2 .

The most chracterized sorption storage materials are listed in Table 13 along with its main properties under given boundary conditions. All the sorption materials listed there make pair with water, as vapour in the case of adsorption and in liquid phase in the case of absorption.

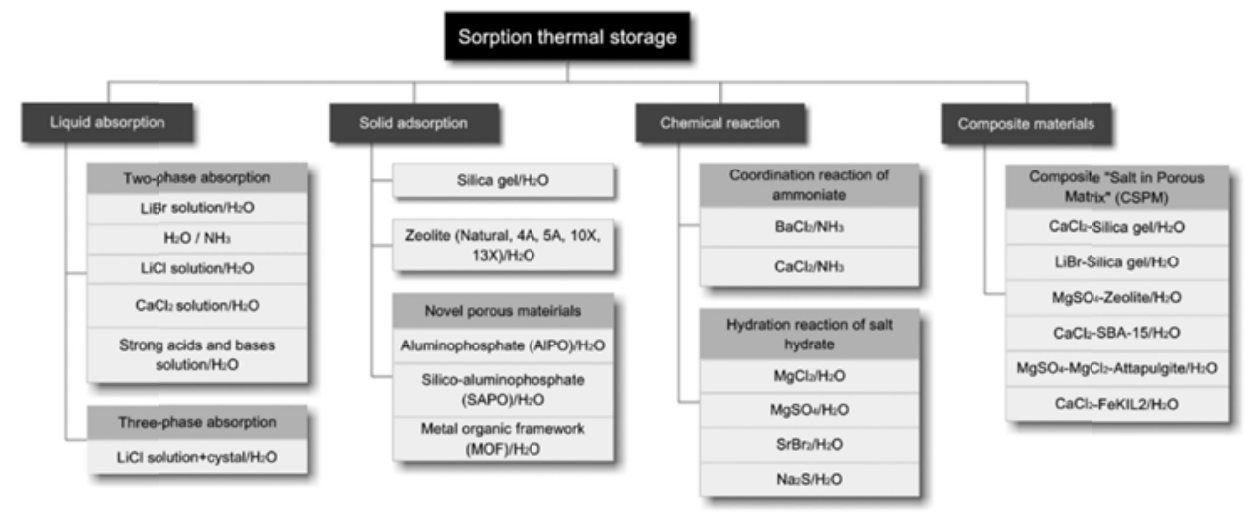

Figure 24. Possible classification of sorption thermal energy storage materials [6]

\subsubsection{Sorption material properties}

Regarding sorption storage materials, main properties requirements are [356]:

- High energy density: to store the maximum energy in the minimum volume.

- Reaction temperature reachable by a solar collector (below $150^{\circ} \mathrm{C}$ ): the heat source to proceed with the endothermic reaction is the sun. 
- Non-toxicity and non-flammability: since will be implemented in buildings and need to accomplish health and safety conditions.

- Non-corrosiveness: especially with the reactor material in contact.

- Stable after several hydration/dehydration reactions: to ensure long term performance of the system.

- Low cost: to be market competitive.

When choosing a sorption material one of the properties that are the first to look at is the energy density given at certain operating conditions, charging/discharging temperature, evaporator/condenser temperature, for closed systems, and ambient temperature. To see how operating conditions are influencing storage capacity Figure 25 is depicted. The first is wanted to be as high as possible in order to store the maximum amount of energy in the lowest volume. Energy densities, under given conditions, and properties of the most common sorption materials are gathered in Table 13.

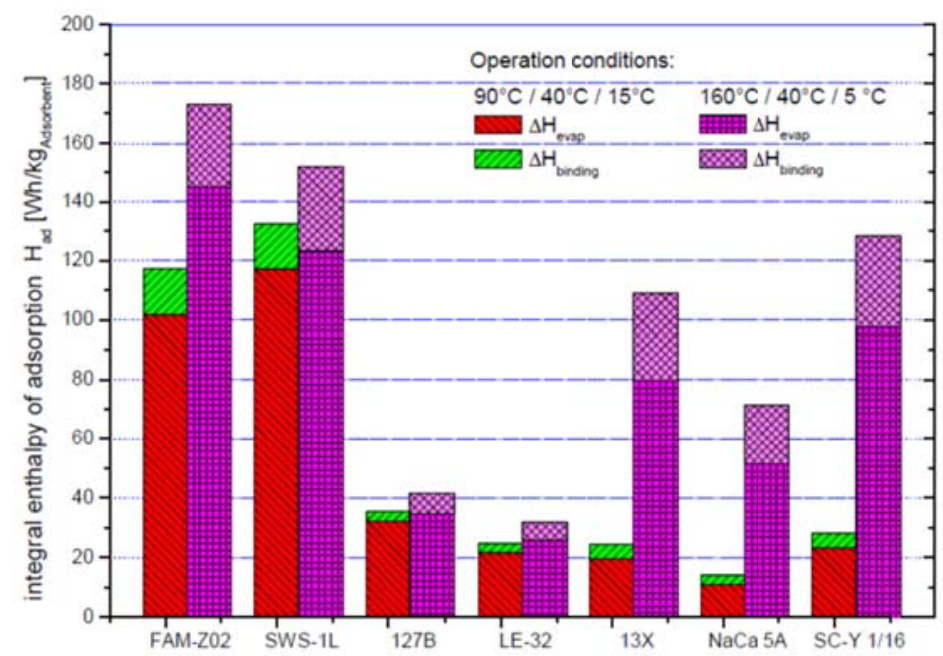

Figure 25. Integral enthalpy od adsorption of different sorption materials under different operating conditions [357]

Another key parameter is the durability and stability that should be examined under repetitive cycles to demonstrate their operability. With an increasing number of thermochemical cycles, a sorption material degrades and its behaviour may change. The stored heat and the overall efficiency of the storage system may decrease over time and numbers of cycles. Also corrosion is one of the drawbacks that sorption materials present and needs to be addressed, especially when working with salt hydrates (chemisorption) and absorption, and being the vessel materials of the containers, heat exchangers, and reactors, metals [357,359]. 
One of the major issues to be overcome in thermochemical energy storage and sorption is the low heat and mass transfer. Then, directly related to heat and mass transfer regarding the sorption materials itself, thermal conductivity and permeability are key parameters for adsorption beds [360,361]. Nevertheless, reactors design should play an important role when enhancing heat and mass transfer [329].

\subsubsection{Physisorption}

\section{a. Zeolites/ $\mathbf{H}_{2} \mathbf{O}$}

Zeolites are alumina silicates with high microporosity. There are synthetic and natural zeolites. A study was conducted comparing natural zeolites and synthetic zeolite $13 \mathrm{X}$ and it was concluded that natural zeolites could be used as a storage material instead of the $13 \mathrm{X}$ synthetic zeolite when the heating temperature is below $100{ }^{\circ} \mathrm{C}$ [362]. For the zeolite $/ \mathrm{H}_{2} \mathrm{O}$ pair, the adsorption heat is about $3300-4200 \mathrm{~kJ} / \mathrm{kg}$, higher than that of silica gel/ $\mathrm{H}_{2} \mathrm{O}$ pair [22]. Additionally, the temperature of desorption can reach high than $200{ }^{\circ} \mathrm{C}$, at which point the zeolite $/ \mathrm{H}_{2} \mathrm{O}$ can be still stable. Zeolites can be employed for different applications as shown in Table 12. Different kinds of crystal cell units of zeolite are shown in Figure 9. The volume of pores for type $\mathrm{X}$ and $\mathrm{Y}$ zeolites, whose void ratio can be as high as $50 \%$ when there is no water adsorbed, is larger than that of other types of pores.

There are other kind of adsorbents sometimes also referred as zeo-like materials because their crystalline structure is somehow similar to the classical zeolites. The two classes that show the most promising features are the aluminophosphates (AlPOs) and the silicoaluminophosphates (SAPOs) [357]. Moreover, MOFS are also being studied and have been mentioned in section 2.2.2 in this review.

\section{b. Silica gel/ $\mathrm{H}_{2} \mathrm{O}$}

The storage density of silica gel is up to four times that of water, being around $2500 \mathrm{~kJ} / \mathrm{kg}$ and the desorption temperature can be very low, but above $50{ }^{\circ} \mathrm{C}[3]$. Desorption temperature should not be higher than $120{ }^{\circ} \mathrm{C}$, and it is usually below $90{ }^{\circ} \mathrm{C}$. Approximately $4-6$ wt.\% water is connected with a single hydroxyl group on the surface of a silica atom to maintain the adsorption capacity. If the desorption temperature is too high (above $120^{\circ} \mathrm{C}$ ), adsorption performance will drop significantly, even to the point of losing its adsorption capacity. In addition, the adsorption quantity of this pair is low, about $0.2 \mathrm{~kg} / \mathrm{kg}$. Silica gel is commercially 
available as pellets or beads of quite spherical shape in diameter from 1 to $4 \mathrm{~mm}$ [361]. Theoretical analysis of a closed adsorption process in a granular silica gel bed through simulations has been published [363] and is concluded that the adsorption time decreases with the particle size until a size range where the value adsorption time is practically constant (in this case, grain sizes in the order of magnitude of $0.1 \mathrm{~mm}$. The minimum adsorption time could then be obtained in this range: optimum diameter of $0.2 \mathrm{~mm}$ and $0.6 \mathrm{~mm}$ for bed thickness of $10 \mathrm{~mm}$ and $40 \mathrm{~mm}$, respectively. Beyond this range, the adsorption time quickly increases with the particle size. This behavior is justified by the change in the limiting adsorption step $[361,363]$.

\subsubsection{Chemisorption}

\section{a. Salt hydrates}

A quite recent review shows an exhaustive screening between 125 salt hydrates to be used as sorption storage material. The results show that for an application that requires a discharging temperature above $60{ }^{\circ} \mathrm{C}, \mathrm{SrBr}_{2} \cdot 6 \mathrm{H}_{2} \mathrm{O}$ and $\mathrm{LaCl}_{3} \cdot 7 \mathrm{H}_{2} \mathrm{O}$ appear to be the most promising, only from thermodynamic point of view. However, the maximum net energy storage density including the water in the water storage tank that they offer (respectively $133 \mathrm{kWh} / \mathrm{m}^{3}$ and 89 $\mathrm{kWh} / \mathrm{m}^{3}$ ) for a classical thermochemical heat storage process are not attractive for the intended application. Furthermore, the thermal efficiency that would result from the storage process based on salt hydrates without condensation heat recovery appears also to be very low (lower than $40 \%$ and typically $25 \%$ ) [364].

Four promising salt hydrates: $\mathrm{MgSO}_{4}, \mathrm{MgCl}_{2}, \mathrm{CaCl}_{2}$, and $\mathrm{Al}_{2}\left(\mathrm{SO}_{4}\right)_{3}$ were proposed for seasonal heat storage of solar energy in the built environment and tested under practical conditions at $150^{\circ} \mathrm{C} / 10-50^{\circ} \mathrm{C} / 10^{\circ} \mathrm{C}$ [365], where $150^{\circ} \mathrm{C}$ was the assumed maximum temperature that can be delivered by a medium temperature collector (vacuum tube) during the summer period and 10 ${ }^{\circ} \mathrm{C}$ was the assumed temperature of the borehole heat exchanger connected to the evaporator/condenser. The performance of the material was assessed by measuring the temperature lift of the bed during the sorption process. The $\mathrm{MgCl}_{2}$ reached a maximum temperature lift of $19{ }^{\circ} \mathrm{C}$ while the $\mathrm{CaCl}_{2}$, the $\mathrm{MgSO}_{4}$, and the $\mathrm{Al}_{2}\left(\mathrm{SO}_{4}\right)_{3}$ reached a maximum temperature lift of $11{ }^{\circ} \mathrm{C}, 4{ }^{\circ} \mathrm{C}$ and $1{ }^{\circ} \mathrm{C}$, respectively. However, the $\mathrm{MgCl}_{2}$ and $\mathrm{CaCl}_{2}$ tend to form a gel-like material during the sorption process due to their hydroscopic nature [341].

The hydration experiments have shown that for both, magnesium and copper sulphate monohydrate, a high water vapour pressure is needed for a sufficiently high reaction rate. The 
reaction of copper sulphate monohydrate with humid air is limited by the equilibrium to a maximum operating temperature of $60{ }^{\circ} \mathrm{C}$. Although higher temperature lifts and operating temperature were observed using $\mathrm{MgSO} 4$, this is not directly correlated with a faster reaction rate. To determine the reaction rate, further research has to be done in micro- and macro-scale to understand the reaction kinetics and mass transfer processes, especially with respect to the different hydrates of magnesium sulphate [366]. Moreover, mixtures of abovementioned salts are being studied at lab scale [367].

\section{b. Composites}

In this section, referred as composites, salt hydrates mixed with additives and combination of physic and chemisorption materials are considered. The mixing of an additive can be done in the sense of enhancing the overall material effective conductivity and thus improve heat transfer, enhance mass transfer by providing higher permeability or to prevent some sorption material drawback which have been experimentally detected such as agglomeration, melting, slow kinetics, etc.

Usually hygroscopic salts present high energy storage density. However, a very slow reaction rate associated with a very low temperature lift in the reactor was observed during the experiments performed. A way to enhance the reaction rate and thus the heat release is to use a physisorption material which might consist on a combination of an active (e.g. zeolite) or a passive (e.g. ceramics), porous, supporting material and salt. The supporting material primarily defines the structure, consistency and form of the storage material. The salt enhances (in the case of an active carrier structure) or defines (in the case of a passive carrier structure) the energy storage capacity of the material [91].

A way to enhance poor heat transfer is to add a high conductive material, such as graphite is by far the most selected additive when developing TCM composites with the purpose of enhancing TCM thermal conductivity. When comparing different host matrices or/and additives, graphite presents the highest conductivity values [333]. For instance, Mauran et al. [368] reported thermal conductivities of about $10-40 \mathrm{~W} / \mathrm{m} \cdot \mathrm{K}$ for $\mathrm{CaCl}_{2}$-expanded natural graphite. Furthermore, expanded graphite prevents agglomeration and raises heat transfer [369]. Composite graphite based TCM are being tested at lab and reactor scale.

Despite the high theoretical energy density of $\mathrm{MgSO}_{4} \cdot 7 \mathrm{H}_{2} \mathrm{O}$ (dense salt: $780 \mathrm{kWh} / \mathrm{m}^{3}$ ), the rate of the reaction and the power delivered is not sufficient for practical use. Therefore, the material needs to be dispersed over a larger exchange surface to react at suitable rate and to release 
greater thermal power. Obviously, this decreases the storage density. Various porous matrixes to disperse this salt in with the aim to optimise the heat transfer or the power density of $\mathrm{MgSO}_{4} \cdot 7 \mathrm{H}_{2} \mathrm{O}$, while preserving the energy density as high as possible, were tested [370]. Composite materials made of magnesium sulphate and sorption materials, such as zeolites and silica gels, were prepared in such a way that the porosity of the sorption materials is preserved. The water vapour can therefore react which both the salt and the matrix. These composite materials are designed with $50 \%$ of the theoretical energy density of the dense salt. The results show that zeolite $/ \mathrm{MgSO}_{4}$ composite is more satisfactory than the others. Indeed, temperature lifts around $30{ }^{\circ} \mathrm{C}$ and maximal power of $28 \mathrm{~mW} / \mathrm{g}$ have been obtained during the hydration of the zeolite/MgSO4 composite.

The $\mathrm{CaCl}_{2}$-silica gel composite has also been extensively studied, the composite containing a 30 wt.\% $\mathrm{CaCl}_{2}$ solution showing a storage capacity of $283 \mathrm{Wh} / \mathrm{kg}$ at the relatively low charging temperature of about $90{ }^{\circ} \mathrm{C}$ [6]. Also, a new composite sorbent $\mathrm{LiNO}_{3} /$ vermiculite (SWS-9V), was specifically developed to operate at low regeneration temperature $\left(<65-70{ }^{\circ} \mathrm{C}\right)$, and demonstrated a remarkable heat storage capacity of $250 \mathrm{Wh} / \mathrm{kg}$ [6].

\section{c. Ammoniated salts}

A solid-gas reaction of ammonium thiocyanate $\left(\mathrm{NH}_{4} \mathrm{SCN}\right)$ and ammonia produces liquid ammoniate $\left(\mathrm{NH}_{4} \mathrm{SCN} \cdot n \mathrm{NH}_{3}\right)$. This system offers a medium for thermal energy storage or a chemical heat pump system [348,371]. Other interesting sorption ammoniated salts are gathered in Figure 26a.

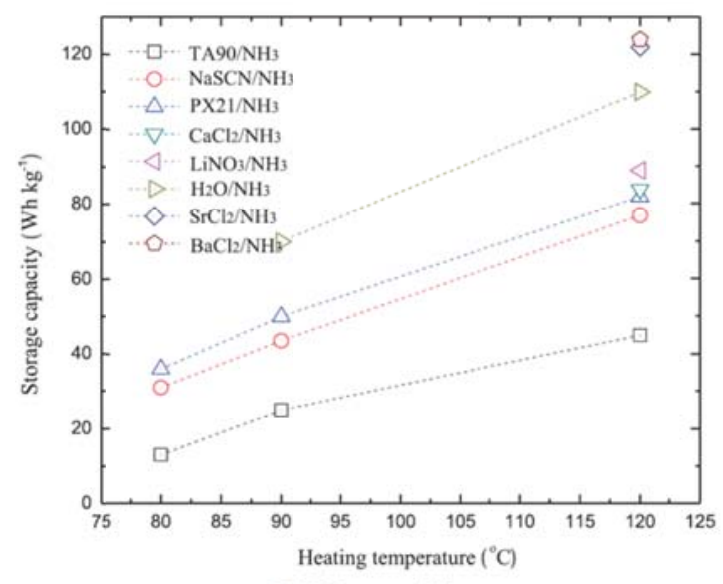

a $\mathrm{NH}_{3}$ as refrigerant

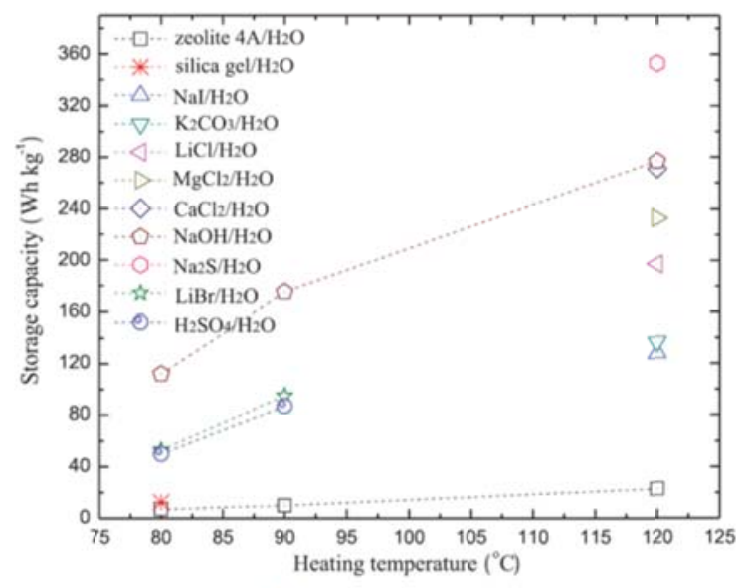

b $\mathrm{H}_{2} \mathrm{O}$ as refrigerant

Figure 26. Storage capacity of different sorption working pairs with ambient temperature of $35^{\circ} \mathrm{C}$ and evaporation temperature of $5^{\circ} \mathrm{C}[3,372]$ 
Thermal properties of working pairs for air conditioning and subzero applications were extracted and summarized as shown in Figure 26 [372]. The summary examines them under the same conditions of an ambient temperature of $35^{\circ} \mathrm{C}$ and evaporation temperature of $5{ }^{\circ} \mathrm{C}$. From the figures above, the most suitable and efficient working pair for the sorption storage system is water and solid/gas reaction used as the refrigerant and sorbent, respectively [3,373].

\subsubsection{Absorption}

The most characterized absorption pairs are listed in Table 13 along with its main properties under given boundary conditions. Among these ones, $\mathrm{NaOH} / \mathrm{H}_{2} \mathrm{O}, \mathrm{LiBr} / \mathrm{H}_{2} \mathrm{O}, \mathrm{LiCl} / \mathrm{H}_{2} \mathrm{O}$, and $\mathrm{CaCl}_{2} / \mathrm{H}_{2} \mathrm{O}$ are the most studied pairs.

The energy density of $\mathrm{NaOH} / \mathrm{H}_{2} \mathrm{O}$ as sorption storage material could be up to $900 \mathrm{MJ} / \mathrm{m}^{3}$ of solution. A low level heat input at $150{ }^{\circ} \mathrm{C}$ was needed to completely charge the system. Analyses show that the concentration ratio of the salt, the corresponding vapor pressure, and the temperature of the discharge are significant parameters. The heat storage capacity of the system is limited by a high temperature needed during the discharge phase [375].

$\mathrm{LiBr} / \mathrm{H}_{2} \mathrm{O}$ is being implemented in single-effect absorption machines which are suitable for solar cooling applications due to good performance at the temperature available from conventional collectors [6]. Static simulations made based on an evaporation temperature of 5$10{ }^{\circ} \mathrm{C}$ to produce heat at $25-33{ }^{\circ} \mathrm{C}$ in winter, show storage density between 180 and $310 \mathrm{kWh} / \mathrm{m}^{3}$ diluted solution [327]. A cascade absorption system with aqueous $\mathrm{LiBr}$ thermal energy storage and R-123 chiller in series were tested. A storage density of $90.9 \mathrm{kWh} / \mathrm{m}^{3}$ was obtained while the volume was based on the volume of a mass concentration of $59 \% \mathrm{LiBr}$ solution [6].

Metal chloride solutions developed for liquid absorption thermal storage mainly include $\mathrm{LiCl}$ and $\mathrm{CaCl}_{2}$, which have been widely applied in open desiccant dehumidification and storage systems [6]. Based on the results presented by ClimateWell (in Sweden) this pair is not suitable for seasonal storage, not because of its storage density $\left(253 \mathrm{kWh} / \mathrm{m}^{3} \mathrm{LiCl}\right.$ salt $)$, which is 2.7 times higher than that of water $\left(25 / 85^{\circ} \mathrm{C}\right)$, but because of the high cost of the salt $(3600 € / \mathrm{m} 3)$ [327] (see Table 12). 
Table 13. Sorption storage materials along with its main properties under given boundary conditions

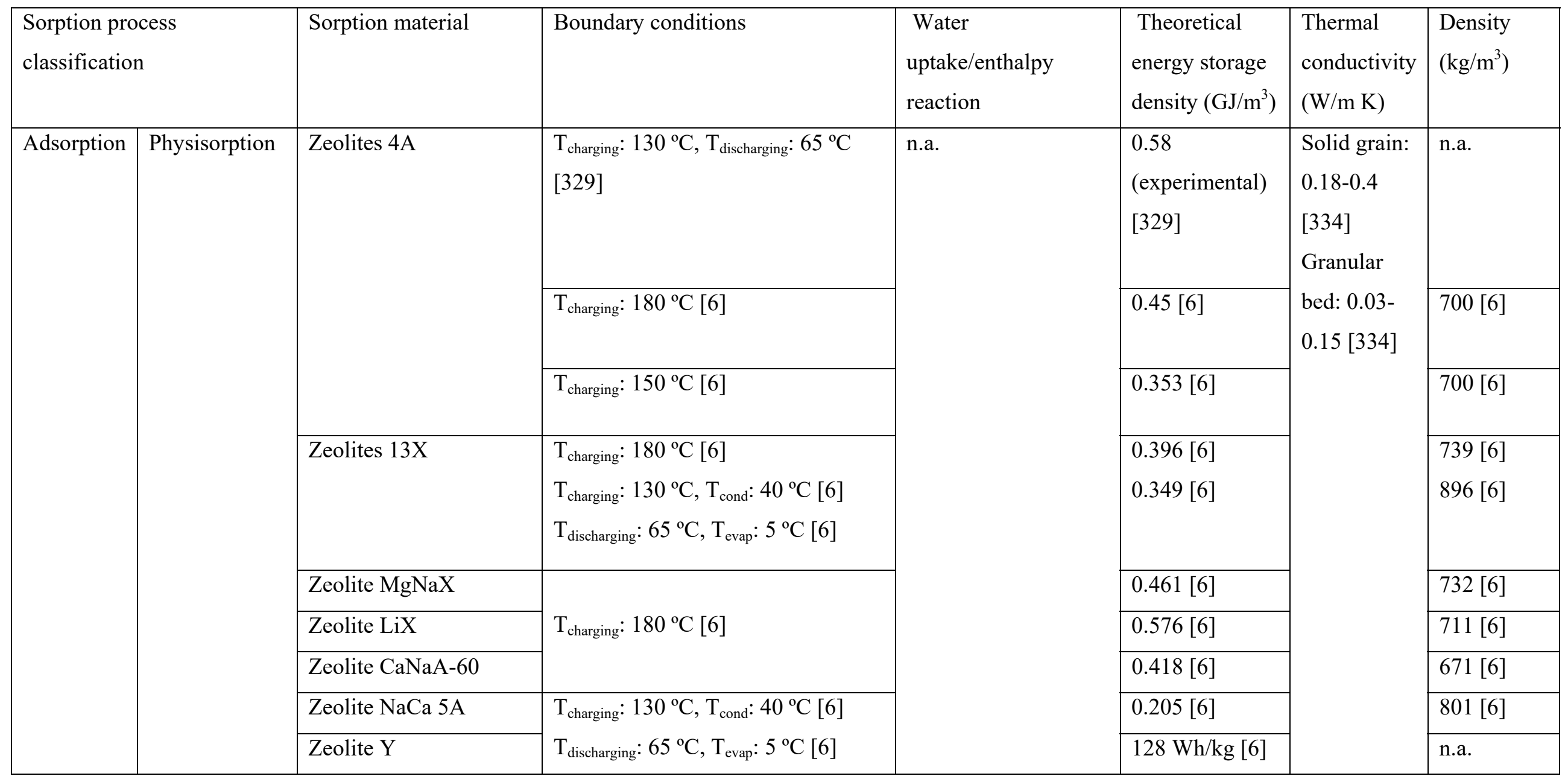




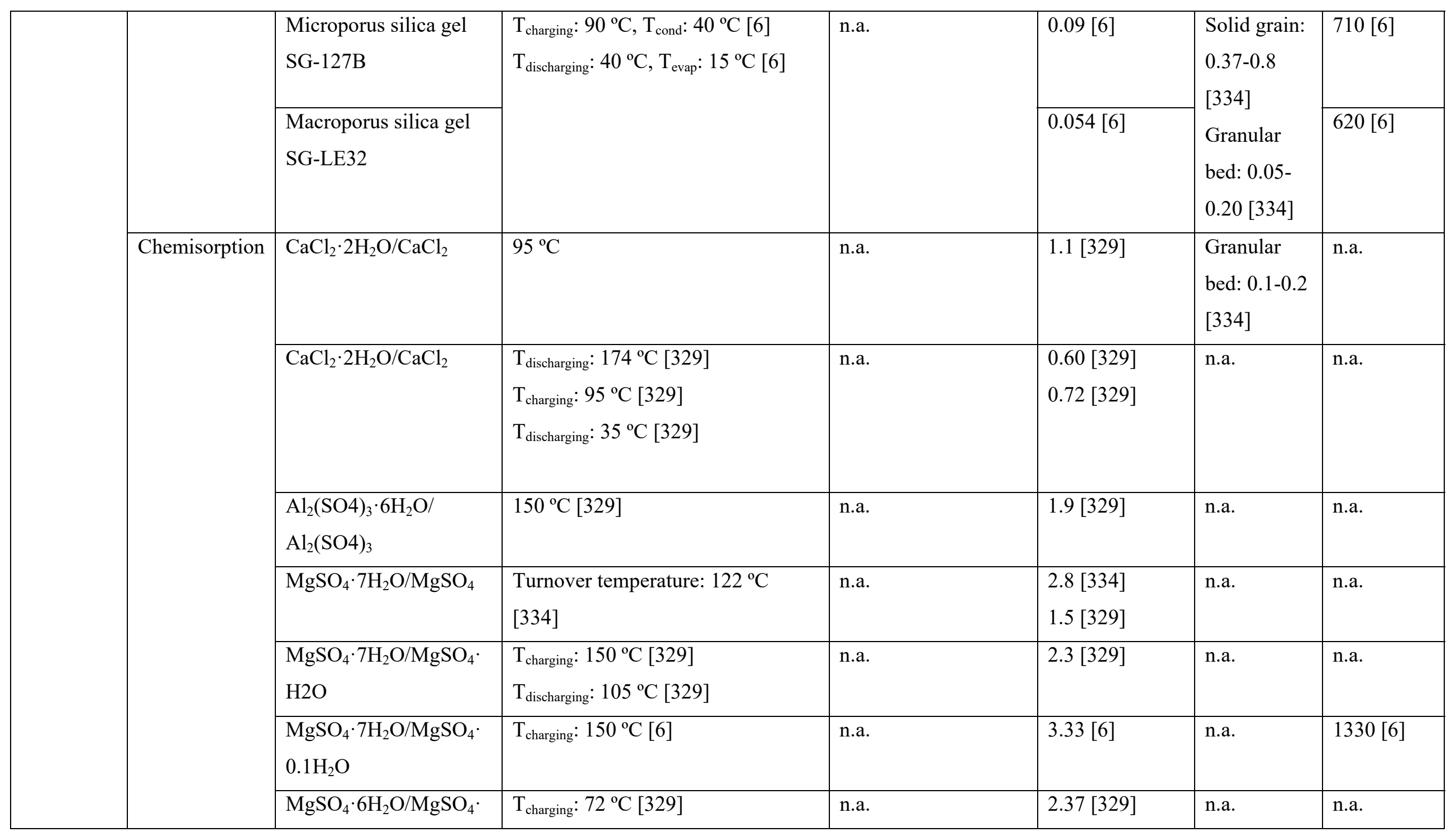




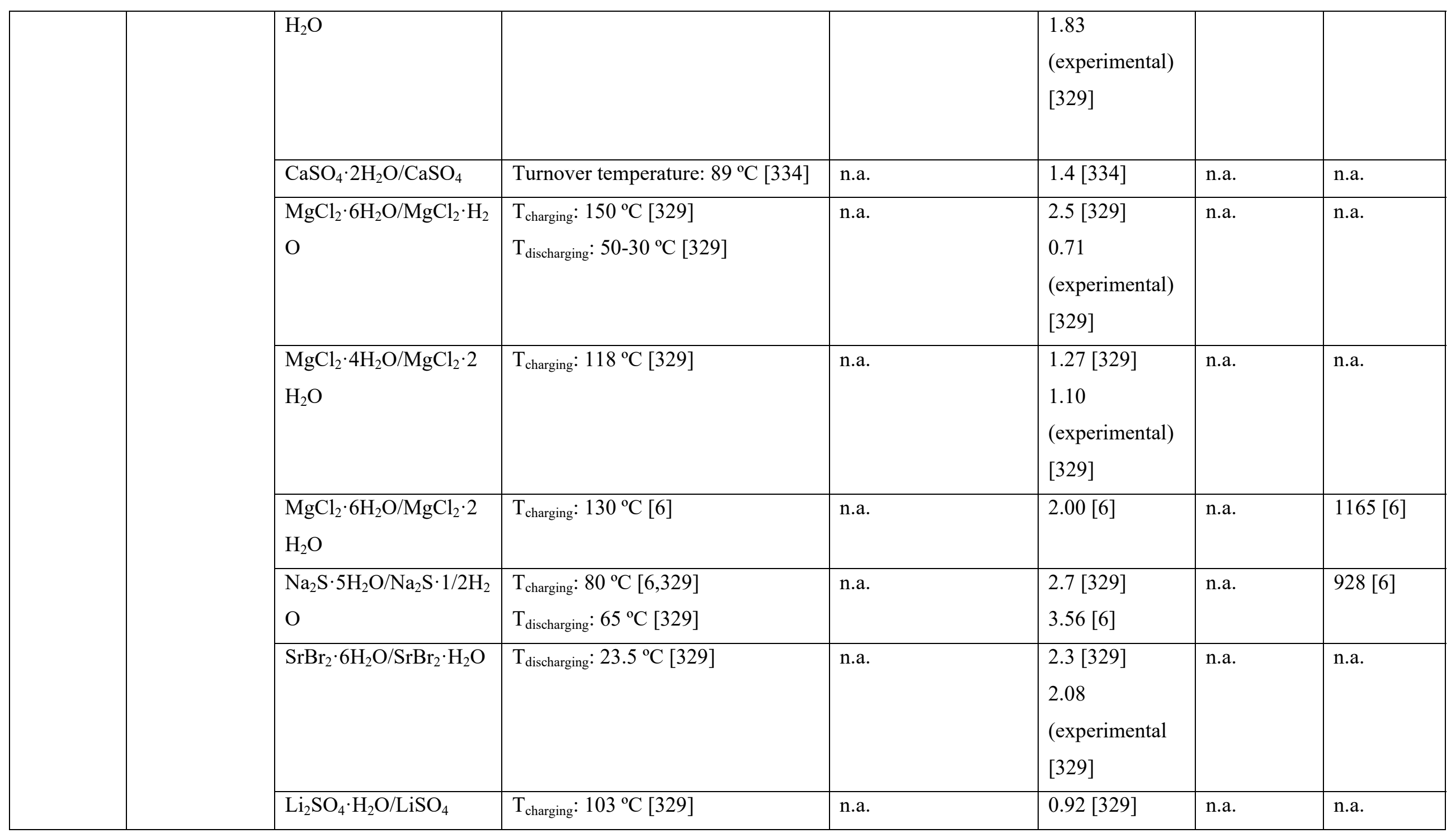




\begin{tabular}{|c|c|c|c|c|c|c|}
\hline & & & & $\begin{array}{l}0.80 \\
\text { (experimental } \\
{[329]}\end{array}$ & & \\
\hline & $\begin{array}{l}\mathrm{CuSO}_{4} \cdot 5 \mathrm{H}_{2} \mathrm{O} / \mathrm{CuSO}_{4} \cdot \mathrm{H} \\
{ }_{2} \mathrm{O}\end{array}$ & $\mathrm{T}_{\text {charging }}: 92^{\circ} \mathrm{C}[329]$ & n.a. & $\begin{array}{l}2.07[329] \\
1.85 \\
\text { (experimental } \\
{[329]}\end{array}$ & n.a. & n.a. \\
\hline \multirow[t]{4}{*}{$\begin{array}{l}\text { Composites: } \\
\text { Inert additives }\end{array}$} & $\begin{array}{l}\mathrm{SrBr}_{2} \cdot 6 \mathrm{H}_{2} \mathrm{O} / \mathrm{SrBr}_{2} \cdot \mathrm{H}_{2} \mathrm{O} \\
+ \text { ENG }\end{array}$ & $\mathrm{T}_{\text {charging: }}: 80^{\circ} \mathrm{C}[6]$ & & $0.63[6]$ & & $1165[6]$ \\
\hline & $\mathrm{CaCl}_{2}+$ ENGP-S-1 & From 100 to $200{ }^{\circ} \mathrm{C}$ & n.a. & n.a. & $1.64[374]$ & $85[374]$ \\
\hline & $\mathrm{CaCl}_{2}+$ ENGP-S-1 & & n.a. & n.a. & $0.74[374]$ & $46[374]$ \\
\hline & $\mathrm{CaCl}_{2}+\mathrm{ACF} \mathrm{VI}$ & & $\begin{array}{l}\text { Dehydration: } 0.10 \\
\mathrm{~g} / \mathrm{g}, 235 \mathrm{~J} / \mathrm{g} \\
\text { Hydration: } 0.12 \mathrm{~g} / \mathrm{g} \text {, } \\
198 \mathrm{~J} / \mathrm{g}\end{array}$ & n.a. & $1.03[374]$ & 649 [374] \\
\hline \multirow{2}{*}{$\begin{array}{l}\text { Composites: } \\
\text { Physisorption } \\
+\end{array}$} & $\begin{array}{l}\text { Aluminosilicate }+30 \% \\
\mathrm{CaCl}_{2}\end{array}$ & $\mathrm{~T}_{\text {charging: }}: 180^{\circ} \mathrm{C}[6]$ & n.a. & $0.62[6]$ & n.a. & $972[6]$ \\
\hline & Mesoporous silica gel + & $\mathrm{T}_{\text {charging }}: 90^{\circ} \mathrm{C}, \mathrm{T}_{\text {cond }}: 40^{\circ} \mathrm{C}[6]$ & n.a. & $0.31[6]$ & n.a. & $646[6]$ \\
\hline
\end{tabular}




\begin{tabular}{|c|c|c|c|c|c|c|c|}
\hline & Chemisorption & $33.7 \% \mathrm{CaCl}_{2}$ & $\mathrm{~T}_{\text {discharging: }}: 40^{\circ} \mathrm{C}, \mathrm{T}_{\text {evap }}: 15^{\circ} \mathrm{C}[6]$ & & & & \\
\hline & & $\begin{array}{l}\text { Zeolite } 13 \mathrm{X}+15 \% \\
\mathrm{MgSO} 4\end{array}$ & $\begin{array}{l}\mathrm{T}_{\text {charging: }}: 150^{\circ} \mathrm{C}[6] \\
\mathrm{T}_{\text {discharging }}: 30^{\circ} \mathrm{C}[6]\end{array}$ & n.a. & $0.60[6]$ & n.a. & $922[6]$ \\
\hline & & $\begin{array}{l}\text { Zeolite } 4 \mathrm{~A}+10 \% \\
\text { MgSO } 4\end{array}$ & $\mathrm{~T}_{\text {charging: }}: 180^{\circ} \mathrm{C}[6]$ & n.a. & $0.64[6]$ & n.a. & $797[6]$ \\
\hline & & Bentonite $+40 \% \mathrm{CaCl}_{2}$ & $\mathrm{~T}_{\text {charging: }}: 150^{\circ} \mathrm{C}[6]$ & n.a. & $0.49[6]$ & n.a. & $695[6]$ \\
\hline & & $\begin{array}{l}\text { Attapulgite }+\mathrm{MgSO}_{4} \\
\text { and } \mathrm{MgCl}_{2} \text { (mass ratio } \\
20 / 80 \text { ) }\end{array}$ & $\begin{array}{l}\mathrm{T}_{\text {charging: }}: 130^{\circ} \mathrm{C}[6] \\
\mathrm{T}_{\text {discharging }}: 30^{\circ} \mathrm{C}[6] \\
85 \% \mathrm{RH}\end{array}$ & n.a. & $442 \mathrm{Wh} / \mathrm{kg}[6]$ & n.a. & n.a. \\
\hline Absorption & & $\mathrm{LiBr} / \mathrm{H}_{2} \mathrm{O}$ & $\begin{array}{l}\text { Without crystallisation } \mathrm{T}_{\text {heat driving: }} \text { : } \\
74{ }^{\circ} \mathrm{C}[3] \\
\text { With crystallisation } \mathrm{T}_{\text {heat driving: }} 93 \\
{ }^{\circ} \mathrm{C}[3] \\
\mathrm{T}_{\text {charging: }}: 72^{\circ} \mathrm{C}[6]\end{array}$ & $\begin{array}{l}\text { Without } \\
\text { crystallisation } 1535 \\
\mathrm{~kJ} / \mathrm{kg}[3] \\
\text { With crystallisation } \\
2068 \mathrm{~kJ} / \mathrm{kg}[3]\end{array}$ & $1.13[6]$ & n.a. & $557[6]$ \\
\hline & & $\mathrm{LiCl} / \mathrm{H}_{2} \mathrm{O}$ & $\begin{array}{l}\text { Without crystallisation } \mathrm{T}_{\text {heat driving: }} \text { : } \\
68^{\circ} \mathrm{C}[3] \\
\text { With crystallisation } \mathrm{T}_{\text {heat driving: }}: 78 \\
{ }^{\circ} \mathrm{C}[3] \\
\mathrm{T}_{\text {charging: }}: 65.6^{\circ} \mathrm{C}[6]\end{array}$ & $\begin{array}{l}\text { Without } \\
\text { crystallisation } 2922 \\
\mathrm{~kJ} / \mathrm{kg}[3] \\
\text { With crystallisation } \\
5271 \mathrm{~kJ} / \mathrm{kg}[3]\end{array}$ & $1.44[6]$ & n.a. & $328[6]$ \\
\hline & & $\mathrm{NaOH} / \mathrm{H}_{2} \mathrm{O}$ & Without crystallisation $\mathrm{T}_{\text {heat driving: }}$ & Without & $0.55[6]$ & n.a. & $355[6]$ \\
\hline
\end{tabular}




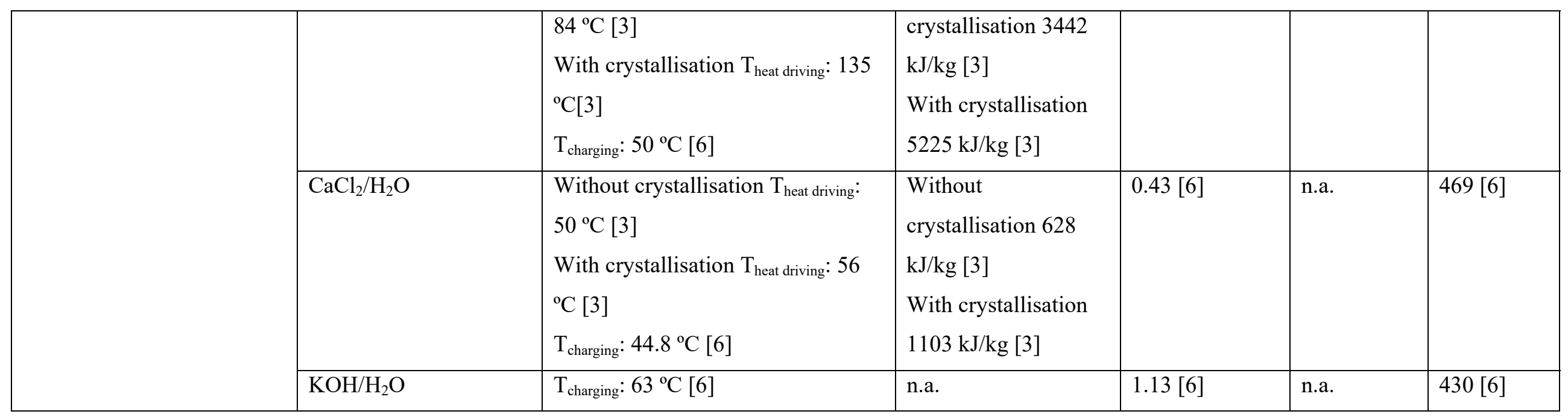




\section{Conclusions}

Sorption has advanced very much due to the immense amount of research carried out around heat pumping and solar refrigeration. Moreover, sorption and thermochemical heat storage attracted considerable attention recently since this technology offers various opportunities in the design of renewable and sustainable energy systems. This paper presents an extensive review of the state of the art of this technology, sorption, both for refrigeration and cooling and for storage, being the first time that both applications are presented together.

The operation principle of the technology is presented and the materials used or in research are listed and compared. The review shots that heat and mass transfer remains currently the main limitations. Mass and heat transfer challenges are common problematic in adsorption, chemical heat pumps and chillers as well as in thermochemical energy storage.

Given that the applications are in different states of development, also today's research is focussed in different aspects. Therefore, absorption heat pumping and refrigeration is today more focussed in the decrease of unit costs and increase of energy efficiency, adsorption is focussed in finding more efficient working pairs, and storage is testing the first prototypes and designing new ones minimizing energy losses throughout all the components, from the storage material to the final consumer

\section{Acknowledgements}

The work is partially funded by the Spanish government (ENE2015-64117-C5-1-R (MINECO/FEDER)). The authors would like to thank the Catalan Government for the quality accreditation given to their research group GREA and DIOPMA (2014 SGR 123, and 2014 SGR 1543, respectively). Dr. Camila Barreneche would like to thank Ministerio de Economia y Competitividad de España for the grant Juan de la Cierva, FJCI-2014-22886. 


\section{References}

1. Y. Fan, L. Luo, B. Souyri. Review of solar sorption refrigeration technologies: Development and applications. Renewable and Sustainable Energy Reviews 11 (2007) $1758-1775$.

2. G.A. Florides, S.A. Tassou, S.A. Kalogirou, L.C. Wrobel. Review of solar and low energy cooling technologies for buildings. Renewable and Sustainable Energy Reviews 6 (2002) 557-572.

3. G. Li, Y. Hwang, R. Radermacher. Review of cold storage materials for air condition application. International Journal of Refrigeration 35 (2012) 2053-2077.

4. A.M. Papadopoulos, S. Oxizidis, N. Kyriakis. Perspectives of solar cooling in view of the developments in the air-conditioning sector. Renewable and Sustainable Energy Reviews 7 (2003) 419-438.

5. I. Sarbu, C. Sebarchievici. General review of solar-powered closed sorption refrigeration. Energy Conversion \& Management 105 (2015) 403-422.

6. N. Yu, R.Z. Wang, L.W. Wang. Sorption thermal storage for solar energy. Progress in Energy and Combustion Science 39 (2013) 489-514.

7. R.E. Critoph. Solid sorption cycles: A short history. International Journal of Refrigeration 35 (2012) 490-493.

8. M. Faraday. On the condensation of several gases into liquids. Philosophical Transactions of the Royal Society of London 113 (1823) 189-198.

9. R.E. Critoph, Y. Zhong. Review of trends in solid sorption refrigeration and heat pumping Technology. Proceedings of the Institution of Mechanical Engineers 219 (2005) 285-300.

10. F.T. Dunsford. Improvements in absorption refrigerating apparatus, GB patent 17 (1951) 672.

11. G. Alefeld. Energiespeicherung durch Heterogenverdampfung (I). Wärme 81 (1975) 89-93.

12. D.I. Tchernev. Exploration of Molecular Sieve Zeolites for Cooling of Buildings with Solar Energy, NSF/RA-770017, Final Report (1977).

13. F. Ziegler. Sorption heat pumping technologies: Comparisions and challenges. International Journal of Refrigeration 32 (2009) 566-576.

14. I. Sarbu, C. Sebarchievici. Review of solar refrigeration and cooling Systems. Energy and Buildings 67 (2013) 286-297.

15. Y. Hwang, R. Randermacher, A. Al-Alili, I. Kubo. Review of solar cooling technology. HVAC\&R Research 14 (2008) 507-28.

16. H.Z. Hassan, A.A. Mohamad. A review on solar cold production through absorption technology. Renewable and Sustainable Energy Reviews 16 (2012) 5331-5348.

17. K. Sumathy, K.H. Yeung, L. Yong. Technology development in the solar adsorption refrigeration Systems. Progress in Energy and Combustion Science 29 (2003) 301-327.

18. T.X. Li, R.Z. Wang, H. Li. Progress in the development of solid-gas sorption refrigeration thermodynamic cycle driven by low-grade thermal energy. Progress in Energy and Combustion Science 40 (2014) 1-58.

19. P. Srikhirin, S. Aphornratana, S. Chungpaibulpatana. A review of absorption refrigeration tecnologies. Renewable and Sustainable Energy Reviews 5 (2001) 343-372.

20. R.Z. Wang, R.G. Oliveira. Adsorption refrigeration-An efficient way to make good use of waste heat and solar energy. Progress in Energy and Combustion Science 32 (2006) 424458.

21. H. Demir, M. Mobedi, S. Ülkü. A review on adsorption heat pump: Problems and solutions. Renewable and Sustainable Energy Reviews 12 (2008) 2381-2403.

22. L.W. Wang, R.Z. Wang, R.G. Oliveira. A review on adsorption working pairs for refrigeration. Renewable and Sustainable Energy Reviews 13 (2009) 518-534. 
23. A.A. Askalany, M. Salem, I.M. Ismael, A.H.H. Ali, M.G. Morsy. A review on adsorption cooling systems with adsorbent carbon. Renewable Sustainable Energy Review 16 (2012) 493-500.

24. S.K. Henninger, F. Jeremias, H. Kummer, C. Janiak. MOFs for use in adsorption heat pump processes. European Journal of Inorganic Chemistry (2012) 2625-2634.

25. D.S. Kim, C.A. Infante Ferreira. Solar refrigeration options-a State-of-the-art review. International Journal of Refrigeration 31 (2008) 3-15.

26. C. Infante Ferreira, D.S. Kim. Techno-economic review of solar cooling technologies based on location-specific data. International Journal of Refrigeration 39 (2014) 23-37.

27. X.Q. Zhai, M. Qu, Y. Li, R.Z. Wang. A review for research and new design options of solar absorption cooling systems. Renewable and Sustainable Energy Reviews 15(2011) 44164423.

28. K.R. Ullah, R. Saidur, H.W. Ping, R.K. Akikur, N.H. Shuvo. A review of solar thermal refrigeration and cooling methods, Renewable and Sustainable Energy Reviews 24 (2013) 490-513.

29. S. Aphornratana. Theoretical and Experimental Investigation of a Combined EjectorAbsorption Refrigerator Department of Mechanical and Process Engineering, University of Sheffield, 1994.

30. S. Arivazhagan, R. Saravanan, S. Renganarayanan. Experimental studies on HFC based two-stage half effect vapour absorption cooling system. Applied Thermal Engineering 26 (2006) 1455-1462.

31. H.B. Gebreslassie, M. Medrano, D. Boer. Exergy analysis of multi-effect water-LiBr absorption systems: from half to triple effect. Renewable Energy 35(2010) 1773-82.

32. M. Lawson, R. Lithgow, G. Vliet. Water-lithium bromide double-effect absorption cooling cycle analysis, ASHRAE Transaction 88 (1982) 811-823.

33. G.P. Xu, Y.Q. Dai, K.W. Tou, C.P. Tso. Theoretical analysis and optimization of a doubleeffect series-flow-type absorption chiller. Applied Thermal Engineering 16(1996) 975-987.

34. M.B. Arun, M.P. Maiya, S.S. Murthy. Performance comparison of double effect parallel flow and series flow water lithium bromide absorption systems. Applied Thermal Engineering 21 (2001) 1273-1279.

35. S.C. Kaushik, A. Arora. Theoretical analysis of $\mathrm{LiBr} / \mathrm{H}_{2} \mathrm{O}$ absorption refrigeration systems. International Journal of Energy Research 33(2009) 1321-1340.

36. R. Gomri. Investigation of the potential of application of single effect and multiple effect absorption cooling systems. Energy Conversion and Management 51(2010)1629-1636.

37. L.G. Farshi, S.M.S. Mahmoudi, M.A. Rosen. Analysis of crystallization risk in double effect absorption refrigeration systems. Applied Thermal Engineering 31 (2011) 1712-1717.

38. ASHRAE Handbook, HVAC Systems and equipment, American Society of Heating, Refrigerating and Air Conditioning Engineers, Atlanta, 2012.

39. K. Sumathy, Z. Huang, Z. Li. Solar absorption cooling with low grade heat source - a strategy of development in South China. Sol Energy 72 (2002)155-165.

40. M. Izquierdo, M. Venegas, P. Rodriguez, A. Lecuona. Crystallization as a limit to develop solar air-cooled $\mathrm{LiBr}-\mathrm{H}_{2} \mathrm{O}$ absorption systems using low-grade heat. Solar Energy Materials \& Solar Cells 81 (2004) 2005-2016.

41. F. Trombe, M. Foex. Production de glace a l'aide de l'energie solaire. International meeting of CNRS, Montlouis, France (1958).

42. G. Grossman. Solar-powered systems for cooling: dehumidification and air conditioning, Solar Energy 72 (2002) 53-62.

43. D. Erickson. Solar icemakers in Maruata Mexico. Solar Today 8 (1994) 21-23.

44. M.J. Tierney. Options for solar-assisted refrigeration trough collectors and double-effect chillers. Renewable Energy 32 (2007) 183-199.

45. I. Sarbu, M. Adam. Applications of solar energy for domestic hot-water and buildings heating/cooling. International Journal of Energy Research 5 (2011) 34-42.

46. H.M.Henning. Solar-assisted Air-conditioning Handbook in Buildings: a Handbook for Planners. Springer-Verlag, Wien, ISBN 3-211-00647-8 (2004). 
47. M. Mokhtar, M.T. Ali, S. Brauniger, A. Afshari, S. Sgouridis, P. Armstrong, M. Chiesa. Systematic comprehensive techno-economic assessment of solar cooling tecnologies using location-specific climate data. Applied Energy 87 (2010) 3766-3778.

48. D.J. Peters. Reversible Greenhouse Climate Control with Desiccant Technique. Report P\&E 2416. Process \& Energy Department, Delft University of Technology, (2010).

49. T. Otanicar, R.A. Taylor, P.E. Phelan. Prospects for solar cooling - an economic and environmental assessment. Solar Energy 86 (2012) 1287-1299.

50. R.M. Lazzarin. Solar cooling: PV or thermal? A thermodynamic and economical analysis. International Journal of Refrigeration 39 (2014) 38-47.

51. S. Du, R.Z. Wang, P. Lin, Z.Z. Xu, Q.W. Pan, S.C. Xu. Experimental studies on an aircooled two-stage $\mathrm{NH}_{3}-\mathrm{H}_{2} \mathrm{O}$ solar absorption air-conditioning prototype. Energy 45 (2012) 581-587.

52. F. Ziegler. State of the art in sorption heat pumping and cooling technologies. International Journal of Refrigeration 25 (2002) 450-459.

53. J. Patek, J. Klomfar. Simple functions for fast calculations of selected thermodynamic properties of the ammonia-water System. International Journal of Refrigeration 18 (1995) 228-234.

54. H. Chua, H. Toh, A. Malek, K. Ng, K. Srinivasan. Improved thermodynamic property field of $\mathrm{LiBr}-\mathrm{H}_{2} \mathrm{O}$ solution. International Journal of Refrigeration 23 (2000) 412-429.

55. F. de'Rossi, R. Mastrullo, P. Mazzei. Working fluids thermodynamic behaviour for vapour compression cicles. Applied Energy 38 (1991) 163-180.

56. A.O. Dieng, R.Z. Wang. Literature review on solar adsorption technologies for ice-making and air conditioning purposes and recent developments in solar technology. Renewable Sustainable Energy Revews 5 (2001) 313-42.

57. F. Ziegler, G. Grossman. Heat-transfer enhancement by additives. International Journal of Refrigeration 19 (1996) 301-309.

58. G.O.G.Löf, R.A. Tybout. The design and cost of optimized systems for residential heating and cooling by solar energy. Solar Energy 16 (1974) 9-18.

59. D.S. Ward, G.O.G. Löf. Design and construction of a residential solar heating and cooling system. Solar Energy 17 (1975) 13-20.

60. D.S. Ward, W.S. Duff, J.C. Ward, G.O.G. Löf. Integration of evacuated tubular solar collectors with lithium bromide absorption cooling systems. Solar Energy 22 (1979) 335341.

61. N. Nakahara, Y. Miyakawa, M. Yamamoto. Experimental study on house cooling and heating with solar energy using flat plate collector. Solar Energy 19 (1977) 657-662.

62. M.R. Yeung, P.K. Yuen, A. Dunn, L.S. Cornish. Performance of a solar-powered air conditioning system in Hong Kong. Solar Energy 48 (1992) 3093-3019.

63. Z.F. Li, K. Sumathy. Experimental studies on a solar powered air conditioning system with partitioned hot water storage tank. Solar Energy 71 (2001) 285-297.

64. J.P. Praene, O. Marc, F. Lucas, F. Miranville. Simulation and experimental investigation of solar absorption cooling System in Reunion Island. Applied Energy 88 (2011) 831-839.

65. D.V. Hattem, P.A. Data. Description of an active solar cooling system, using a $\mathrm{LiBr}-\mathrm{H}_{2} \mathrm{O}$ absorption machine. Energy and Building 3 (1981) 169-196.

66. A. Al-Karaghouli, I. Abood, N.I. Al-Hamdani. The solar energy research center building thermal performance evaluation during the summer season. Energy Conversion and Management 32 (1991) 409-417.

67. R. Best, N. Ortega. Solar refrigeration and cooling. Renewable Energy 16 (1999) 685-690.

68. M. Izquierdo, A. Syed, P. Rodriguez, G. Maidment, J. Missenden, A. Lecuona, R. Tozer. A novel experimental investigation of a solar cooling system in Madrid. International Journal of Refrigeration 28 (2005) 859-871.

69. A. Mammoli, P. Vorobieff, H. Barsun, R. Burnett, D. Fisher. Energetic, economic and environmental performance of a solar-thermal-assisted HVAC system. Energy Buildings 42 (2010) 1524-1535. 
70. R. Lizarte, M. Izquierdo, J.D. Marcos, E. Palacios. An innovative solar-driven directly aircooled $\mathrm{LiBr}-\mathrm{H}_{2} \mathrm{O}$ absorption chiller prototype for residential use. Energy Buildings 47 (2012) $1-11$.

71. F. Agyenim, I. Knight, M. Rhodes. Design and experimental testing of the performance of an outdoor $\mathrm{LiBr} / \mathrm{H}_{2} \mathrm{O}$ solar thermal absorption cooling System with a cold store. Solar Energy 84 (2010) 735-744.

72. M. Balghouthi, M.H. Chahbani, A. Guizani. Feasibility of solar absorption air conditioning in Tunisia. Buildings and Environment 43 (2008) 1459-1470.

73. F. Storkenmaier, M. Harm, C. Schweigler, F. Ziegler, J. Albers, P. Kohlenbach, T. Sengewald. Small-capacity water $/ \mathrm{LiBr}$ absorption chiller for solar cooling and waste-heat driven cooling. In: Proceedings of International Congress of Refrigeration, Washington D.C., USA (2003).

74. A. Kühn, M. Harm, P. Kohlenbach, S. Petersen, C. Schweigler, F. Ziegler. Betriebsverhalten einer $10 \mathrm{~kW}$ Absorptionskälteanlage für solare Kühlung. KI Luft- und Kältetechnik 7 (2005) 263-266.

75. M. Safarik, L. Richter, F. Mockel, S. Kretschmar. Performance data of a small capacity absorption chiller. In: Proceedings of International Conference Solar Air-conditioning, Bad Staffelstein, Germany, (2005).

76. T. Ishibashi. The operation results of the Yazaki experimental solar house. In: Silver Jubilee Congress of ISES, Atlanta, USA, (1979).

77. P. Lamp, F. Ziegler. European research on solar-assisted air conditioning. International Journal of Refrigeration 21 (1998) 89-99.

78. A. Lokurlu, G. Müller. Experiences with the worldwide first solar cooling system based on trough collectors combined with double effect absorption chillers. In: Proceedings of International Conference Solar Air-conditioning, Bad Staffelstein, Germany, (2005).

79. F. Gutiérrez. Behaviour of a household absorption-diffusion refrigerator adapted to autonomous solar operation. Solar Energy 40 (1988) 17-23.

80. G. Kunze. Efficient solar cooling with an improved ammonia-absorption system. Renewable Energy World 3 (2000) 111-112.

81. U. Jakob, U. Eicker, A.H.Taki, M.J. Cook. Development of an optimized solar driven diffusion-absorption cooling machine. In: Proceedings of ISES Solar World Congress, Göteborg, Sweden, (2003).

82. Y. Shiran, A. Shitzer, D. Degani. Computerized design and economic evaluation of an aquaammonia solar operated absorption system. Solar Energy 29 (1982) 43-54.

83. M.O. McLinden, S.A. Klein. Simulation of an absorption heat pump solar heating and cooling system. Solar Energy 31 (1983) 473-482.

84. S.G. Alvares, Ch. Trepp. Simulation of a solar driven aqua-ammonia absorption refrigeration system, part 2: viability for milk cooling at remote Brazilian dairy farms. International Journal of Refrigeration 10 (1987) 70-76.

85. R. Best. Experimental studies on the operating characteristics of an ammonia-water absorption system for cooling. Chemical Engineering Research and Design 69 (1991) 153160.

86. ARTISC. Refrigeration, heating and air-conditioning using an absorption refrigeration system heated by transparently insulated solar collectors. EU project, Fifth Framework Program, CRAFT/70436/1999, (2003).

87. U. Jakob, U. Eicker, D. Schneider, A.H. Taki, M.J. Cook. Simulation and experi- mental investigation into diffusion absorption cooling machines for air- conditioning applications. Applied Thermal Engineering 28 (2008) 1138-1150.

88. F.Z. Sierra, R. Best, F.A. Holland. Experiments on an absorption refrigeration system powered by a solar pond. Heat Recovery Systems and CHP 13 (1993): 401-408.

89. SACE. Solar air conditioning in Europe: evaluation report. EC project NNE5/2001/25, (2003).

90. L. Richter, M. Safarik. Solar cooling with ammonia water absorption chillers. In: Proceedings of International Conference Solar Air conditioning, Bad Staffelstein, Germany, (2005). 
91. S.A.M. Said, M.A.I. El-Shaarawi, M.U. Siddiqui. Alternative designs for a 24-h operating solar-powered absorption refrigeration technology. International Journal of Refrigeration 35 (2012) 1967-1977.

92. A. Al/Alili, M.D. Islam, I. Kubo, Y. Hwang, R. Radermacher. Modeling of a solar powered absorption cycle for Abu Dhabi. Applied Energy 93 (2012) 160-167.

93. P. Worsøe-Schmidt. A solar-powered solid-absorption refrigeration system. International Journal of Refrigeration 2 (1979) 75-82.

94. A. Erhard, E. Hahne. Test and simulation of a solar-powered absorption cooling machine. Solar Energy 59 (1997) 155-162.

95. N.K. Bansal, J. Blumenberg, H.J. Kavasch, T. Roettinger. Performance testing and evaluation of solid absorption solar cooling unit. Solar Energy 61 (1997) 127-140.

96. M. Medrano, M. Bourouis, A. Coronas. Double-lift absorption refrigeration cycles driven by low-temperature heat sources using organic fluid mixtures as working pairs. Applied Energy 68 (2001) 173-185.

97. D. Boer, M. Valles, A. Coronas. Performance of double effect absorption compression cycles for air-conditioning using methanol-TEGDME and TFE-TEGDME systems as working pairs. International Journal of Refrigeration 21(1998) 542-555.

98. R.J. Romero, W. Rivera, I. Pilatowsky, R. Best. Comparison of the modeling of a solar absorption system for simultaneous cooling and heating operating with an aqueous ternary hydroxide and with water/lithium bromide. Solar Energy Materials and Solar Cells 70 (2001) 301-308.

99. C.O. Rivera, W. Rivera. Modeling of an intermittent solar absorption refrigeration system operating with ammonia/lithium nitrate mixture. Solar Energy Materials and Solar Cells 76 (2003) 417-427.

100. S. Steiu, D. Salavera, J.C. Bruno, A. Coronas. A basis for the development of new ammonia-water-sodium hydroxide absorption chillers. International Journal of Refrigeration 32 (2009) 577-587.

101. X. Zhang, D. Hu. Performance simulation of the absorption chiller using water and ionic liquid 1-ethyl-3-methylimidazolium dimethylphosphate as the working pair. Applied Thermal Engineering 31 (2011) 3316-3321.

102. J. Ren, Z. Zhao, X. Zhang. Vapor pressures, excess enthalpies, and specific heat capacities of the binary working pairs containing the ionic liquid 1-ethyl-3methylimidazolium dimethylphosphate. Journal of Chemical Thermodynamics 43 (2011) $576-583$.

103. G. Zuo, Z. Zhao, S. Yan, X. Zhang. Thermodynamic properties of a new working pair: 1-ethyl-3-methylimidazolium ethylsulfate and water. Chemical Engineering Journal 156 (2010) 613-617.

104. A.N. Abdelmessih, M. Abbas, A. Al-Hashem, J. Munson. Ethylene glycol/water as working fluids for an experimental absorption cycle. Experimental Heat Transfer 20 (2007) 87-102.

105. R.J. Romero, L. Guillen, I. Pilatowsky. Monomethylamine-water vapour absorption refrigeration system. Applied Thermal Engineering 25 (2005) 867-876.

106. R.E. Critoph, S.J. Metcalf. Specific cooling power intensification límits in ammoniacarbon adsorption refrigeration systems. Applied Thermal Engineering 24 (2004) 661-678.

107. B.B. Saha, S. Koyama, J.B. Lee, K. Kuwahara, K.C.A. Alam, Y. Hamamoto, A. Akisawa, T. Kashiwagi. Performance evaluation of a low-temperature waste heat driven multi-bed adsorption chiller. International Journal of Multiphase Flow 29 (2003) 12491263.

108. H.T. Chua, K.C.. Ng, A. Malek, T. Kashiwagi, T. Akisawa, B.B. Saha. Modeling the performance of two-bed, sillica gel-water adsorption chillers. International Journal of Refrigeration 22 (1999) 194-204.

109. D.C. Wang, Z.Z. Xia, J.Y. Wu, R.Z. Wang, H. Zhai, W.D. Dou. Study of a novel silica gel-water adsorption chiller. Part I. Design and performance prediction. International Journal of Refrigeration 28 (2005) 1073-1083. 
110. O.C. Iloeje, A.N. Ndili, S.O. Enibe. Computer simulation of a $\mathrm{CaCl}_{2}$ solid-adsorption solar refrigerator. Energy 20 (1995) 1141-1151.

111. Y. Kato, M. Yamada, T. Kanie, Y. Yoshizawa. Calcium oxide/carbon dioxide reactivity in a packed bed reactor of a chemical heat pump for high-temperature gas reactors. Nuclear Engineering and Design 210 (2001) 1-8.

112. R.E. Critoph. Rapid cycling solar/biomass powered adsorption refrigeration system. Renewable Energy 16 (1999) 673-678.

113. Q.W. Pan, R.Z. Wang, L.W. Wang, D.D. Liu. Design and experimental study of a silica gel-water adsorption chiller with modular adsorbers. International Journal of Refrigeration 67 (2016) 336-344.

114. Wang LW, Wang RZ, Lu ZS, Chen CJ, Wu JY. Comparison of the adsorption performance of compound adsorbent in a refrigeration cycle with and without mass recovery. Chemical Engineering Science 61 (2006) 3761-3770.

115. Y.H. Zhang. Adsorption function. Shanghai, China: Publishing House of Scientific and Technological Literature in Shanghai; 1989.

116. R.P.H. Gasser. The chemical adsorption and catalysis of metal. Beijing, China: Publishing House of Beijing University; 1991 [in Chinese].

117. L.W. Wang, R.Z. Wang, J.Y. Wu, K. Wang, S.G. Wang. Adsorption ice makers for fishing boats driven by the exhaust heat from diesel engine: choice of adsorption pair. Energy Conversion \& Management 45 (2004) 2043-2057.

118. L.W. Wang, R.Z. Wang, J.Y. Wu, K. Wang. Compound adsorbent for adsorption ice maker on fishing boats. International Journal of Refrigeration 27 (2004) 401-408.

119. M. Groll. Reaction beds for dry sorption machines. In: Proceedings of the symposium of solid sorption refrigeration; 1992. p. 208-14.

120. C. Coste, G. Crozat, S. Mauran. Gaseous-solid reaction. EUA Patent 4,595,774; 1986.

121. S. Mauran, P. Prades, F.L. Haridon. Heat and mass transfer in consolidated reaction beds for thermochemical systems. Heat Recovery Systems and CHP 13 (1993) 315-319.

122. L.L: Vasiliev, D.A. Mishkinis, L.L. Vasiliev Jr. Multi-effect complex compound/ammonia sorption machines. In: Proceedings of the sorption heat pump conferences, vol. I; 1996. p. 3-8.

123. Z. Aidoun, M. Ternan. Salt impregnated carbon fibres as the reactive medium in a chemical heat pump: the $\mathrm{NH}_{3}-\mathrm{CoCl}_{2}$ system. Applied Thermal Engineering 22 (2002) $1163-1173$.

124. K. Wang, J.Y. Wu, R.Z. Wang, L.W. Wang. Effective thermal conductivity of expanded graphite- $\mathrm{CaCl}_{2}$ composite adsorbent for chemical adsorption chillers. Energy Conversion \& Management 47 (2006) 1902-1912.

125. R.T. Yang. Gas separation by adsorption methods. Beijing, China: Publishing House of Chemical Industry; 1991 [in Chinese].

126. Y. Zhong, R.E. Critoph, R.N. Thorpe, Z. Tamainot-Telto, Y.I. Aristov. Isothermal sorption characteristics of the $\mathrm{BaCl}_{2}-\mathrm{NH}_{3}$ pair in a vermiculite host matrix. Applied Thermal Engineering 27 (2007) 2455-2462.

127. Peng SP, Wang B, Luo ZJ. Structure of atoms and molecules, complex compound colloid chemistry. Chengdou, China: People's Publishing House of Sichuan; 1984 [in Chinese].

128. Y. Kato, F. Takahashi, A. Watanabe, Y. Yoshizawa. Thermal analysis of a magnesium oxide/water chemical heat pump for cogeneration. Applied Thermal Engineering 21 (2001) $1067-1081$.

129. Y. Kato, Y. Sasaki, Y. Yoshizawa. Magnesium oxide/water chemical heat pump to enhance energy utilization of a cogeneration system. Energy 30 (2005) 2144-2155.

130. T. Kiyoyama. Metal oxides and their catalysis. Beijing, China: Publishing House of Chemical Industry; 1991 [in Chinese].

131. J.H. Han, K.N. Lee. Gas permeability of expanded graphite-metallic salt composite. Applied Thermal Engineering 21 (2001) 453-463.

132. L. Pino, Y.I. Aristov, G. Cacciola, G. Restuccia. Composite materials based on zeolite 4A for adsorption heat pumps. Adsorption 3 (1996) 33-40. 
133. C.J. Chen, R.Z. Wang, Z.Z. Xia, J.K. Kiplagat, Z.S. Lu. Study on a compact silica gelwater adsorption chiller without vacuum valves: design and experimental study. Applied Energy 87 (2010) 2673-2681.

134. R.J.H. Grisel, S.F. Smeding, R. de Boer. Waste heat driven silica gel/water adsorption cooling in trigeneration. Applied Thermal Engineering 30 (2010) 1039-1046.

135. W. Dechang, Z. Jipeng, X. Yanzhi, H. Yanpei, W. Shuwei. Investigation of adsorption performance deterioration in silica gel-water adsorption refrigeration. Energy Conversion Management 58 (2012) 157-162.

136. M. Pons, F. Poyelle. Adsorptive machines with advantaged cycles for heat pumping or cooling applications. International Journal of Refrigeration 22 (1999) 27-37.

137. A. Akahira,K.C.A. Alam, Y. Hamamoto, A. Akisawa, T. Kashiwagi. Experimental investigation of mass recovery adsorption refrigeration cycle. International Journal of Refrigeration 28 (2005) 565-572.

138. D.C. Wang, J.Y. Wu, Z.Z. Xia, H. Zhai, R.Z. Wang, W.D. Dou. Study of a novel silica gel-water adsorption chiller. Part II. Experimental study. International Journal of Refrigeration 28 (2005) 1084-1091.

139. B.B. Saha, A. Akisawa, T. Kashiwagi. Solar/waste heat driven two-stage adsorption chiller: the prototype. Renewable Energy 23 (2001) 93-101.

140. T. Nuñez, W. Mittelbach, H.M. Henning. Development of an adsorption chiller and heat pump for domestic heating and air-conditioning applications. In: Proc. of Int. Conf. on Heat Powered Cycles, Cyprus, 2004.

141. K. Van den Heuvel. Hybrid Adsorption Compression Heat Pump Systems. Report P\&E 2402. Process \& Energy Department, 2010.

142. C.H. Li, R.Z. Wang, Y.J. Dai. Simulation and economic analysis of a solar-powered adsorption refrigerator using an evacuated tube for thermal insulation. Renewable Energy 28 (2003) 249-269.

143. L.H. Luo, R.Z. Wang, Y.J. Dai, J.Y. Wu, J.M. Shenand, B.B. Zhang. An efficient solar powered adsorption chiller and its application in low temperature grain storage. Solar Energy 81 (2007) 607-613.

144. Y. Hamamoto, K.C. Amanul Alam, A. Akisawa, T. Kashiwagi. Performance evaluation of a two-stage adsorption refrigeration cycle with different mass ratio. International Journal of Refrigeration 28 (2005) 344-352.

145. M.Z.I. Khan, K.C.A. Alam, B.B. Saha, Y. Hamamoto, A. Akisawa, T. Kashiwagi. Parametric study of a two-stage adsorption chiller using re-heat the effect of overall thermal conductance and adsorbent mass on System performance. Internatinal Journal of Thermal Science 45 (2005) 511-519.

146. A. Sakoda, M. Suzuki. Fundamental study on solar powered adsorption cooling system. Journal of Chemical Engineering of Japan 17 (1984) 52-57.

147. H. Henning, H. Glaser. Solar assisted adsorption system for a laboratory of the university Freiburg.

148. M.A. Tahat. Heat-pump/energy-store using silica gel and water as a working pair. Applied Energy 69 (2001) 19-27.

149. H.M. Henning, E. Wiemken. Solar assisted air conditioning of buildings overview on technologies and state of the art. In: Proceedings of ISES Solar World Congress. Gothenburg, Sweden, 2003.

150. T. Nuñez, W. Mittelbach, M.H. Henning. Development of a small capacity adsorption system for heating and cooling applications. In: Proceedings of the international sorption heat pump conference, Denver, USA, 2005.

151. R. De Boer, F.S. Smeding, H.J.R. Grisel. Development and testing of a sorbent filled heat exchanger for use in compact solid sorption cooling systems. In: Proceedings of the international sorption heat pump conference, Denver, USA, 2005.

152. Y. Hamamoto, A. Akisawa, T. Kashiwagi. Study of a hybrid desiccant cooling system combined with a two-stage adsorption chiller. In: Proceedings of the international sorption heat pump conference, Denver, USA, 2005. 
153. C.D. Wang, Y.J. Wu, Z.R. Wang, Z.Z. Xia. Research on a novel adsorption chiller driven by low grade heat source. In: Proceedings of the international sorption heat pump conference, Denver, USA, 2005.

154. Z.R. Wang, Q.X. Kong, Y.J. Wu, Y. Huangfu, W.D.Wu. Performance research of a micro cchp system with adsorption chiller. In: Proceedings of the international sorption heat pump conference, Denver, USA, 2005.

155. J. Wu, D. Wang, X. Zhai, R. Wang. Research on control method of the adsorption chiller driven by a variable heat source - solar energy. In: Proceedings of the international sorption heat pump conference, Denver, USA, 2005.

156. C. Choi, S. Jeong. Experimental study on the development of micro adsorption refrigerator. In: Proceedings of the international sorption heat pump conference, Denver, USA, 2005.

157. C. Hildbrand, P. Dind, M. Pons, F. Buchter. A new solar powered adsorption refrigerator with high performance. Solar Energy 77 (2004) 311-318.

158. Z.Z. Xia, R.Z. Wang, J.Y. Wu, D.C. Wang. New type of effective adsorption chiller adopt separate heat pipe. China patent 200410025398.0, 2004.

159. G. Restuccia, A. Freni, S. Vasta, Y. Aristov. Selective water sorbent for solid sorption chiller: experimental results and modelling. International Journal of Refrigeration 27 (2004) 284-293.

160. Y.L. Liu, R.Z. Wang, Z.Z. Xia. Experimental study on a continuous adsorption water chiller with novel design. International Journal of Refrigeration 28 (2005) 218-230.

161. N. Dous, F. Meunier. Experimental study of cascading adsorption cycles. Chemical Engineering Science 44 (1989) 225-235.

162. H. Peng, Y. Juan-Juan, C. Ze-Shao. Analysis for composite zeolite/foam aluminumwater mass recovery adsorption refrigeration system driven by engine exhaust heat. Energy Conversion \& Management 50 (2009) 255-261.

163. M.Z.I. Khan, B.B. Saha, K.C.A. Alam, A. Akisawa, T. Kashiwagi. Study on solar/waste heat driven multi-bed adsorption chiller with mass recovery. Renewable Energy 23 (2007) 365-381.

164. G.Z. Yang, Z.Z. Xia, R.Z. Wang. Research on a compact adsorption room air conditioner. Energy Conversion \& Management 47 (2006) 2167-2177.

165. K.C. Ng, X.L. Wang, Y.S. Lim, B.B. Saha, A. Chakarborty, S. Koyama S, A. Akisawa, T. Kashiwagi. Experimental study on performance improvement of a four-bed adsorption chiller by using heat and mass recovery. International Journal of Heat \& Mass Transfer 49 (2006) 3343-3348.

166. K.C.A. Alam, B.B. Saha, A. Akisawa, T. Kashiwagi. Influence of design and operating conditions on the system performances of a two-stage adsorption chiller. Chemical Engineering Communication 191 (2004) 981-997.

167. S.K. Farid, M.M. Billah, M.Z.I. Khan, M.M. Rahman, U.M. Sharif. A numerical analysis of cooling water temperature of two-stage adsorption chiller along with different mass ratios. International Commun Heat and Mass Transfer 38 (2011) 1086-1092.

168. B.B. Saha, C. Boelman, T. Kashiwagi. Computational analysis of an advanced adsorption-refrigeration cycle. Energy 20 (1995) 983-994.

169. B.B. Saha, S. Koyama, T. Kashiwagi, A. Akisawa, K.C. Ng, H.T. Chua. Waste heat driven dual-mode, multi-stage, multi-bed regenerative adsorption system. International Journal of Refrigeration 26 (2003) 749-757.

170. B.B. Saha, S. Koyama, K.C. Ng, Y. Hamamoto, A. Akisawa, T. Kashiwagi. Study on a dual-mode, multi-stage, multi-bed regenerative adsorption chiller. Renewable Energy 31 (2006) 2076-2090.

171. M.Z.I. Khan, K.C.A. Alam, B.B. Saha, A. Akisawa, T. Kashiwagi. Performance evaluation of multi-stage, multi-bed adsorption chiller employing re-heat scheme. Renewable Energy 33 (2008) 88-98.

172. A. Hauer. Evaluation of adsorbent materials for heat pump and thermal energy storage applications in open systems. In: Proceedings of the international sorption heat pump conference, Denver, USA, 2005. 
173. Y.I. Aristov, G. Restuccia, G. Cacciola, V.N. Parmon. A family of new working materials for solid sorption air conditioning systems. Applied Thermal Engineering 22 (2002) 191-204.

174. K. Oertel, U. Sprengel, S. Mande, P. Ghosh, V.V.N. Kishore. Development of an advanced solar-hybrid adsorption cooling system for decentralized storage of agricultural products in India. DLR-TERI joint project report. The Commission of the European Communities, DG-XII, 1996.

175. L.G. Gordeeva, A.D. Grekova, T.A. Krieger, Y.I. Aristov. Adsorption properties of composite materials ( $\mathrm{LiClpLiBr}) /$ silica. Microporous Mesoporous Materials 126 (2009) 262-267.

176. G. Maggio, L.G. Gordeeva, A. Freni, Y.I. Aristov, G. Santori, F. Polonara, G. Restuccia. Simulation of a solid sorption ice-maker based on the novel composite sorbent lithium chloride in silica gel pores. Applied Thermal Engineering 29 (2009) 1714-1720.

177. K.F. Fong, T.T. Chow, C.K. Lee, Z. Lin, L.S. Chan. Advancement of solar desiccant cooling system for Building use in subtropical Hong Kong. Energy and Buildings 42 (2010) 2386-2399.

178. I. Solmus, C. Yamal, B. Kaftanoglu, D. Baker, A. Caglar. Adsorption properties of a natural zeolite-water pair for use in adsorption cooling cycles. Applied Energy 87 (2010) 2062-2067.

179. D.C. Wang, Z.Z. Xia, J.Y. Wu. Design and performance prediction of a novel zeolitewater adsorption air conditioner. Energy Conversion \& Management 47 (2006) 590-610.

180. S. Vasta, A. Freni, A. Sapienza, F. Costa, G. Restuccia. Development and lab-test of a mobile adsorption air-conditioner. International Journal of Refrigeration 35 (2012) 701708.

181. S. Szarzynski, Y. Feng, M. Pons. Study of different internal vapour transports for adsorption cycles with heat regeneration. International Journal of Refrigeration 20 (1997) 390-401.

182. H.M. Lai. An enhanced adsorption cycle operated by periodic reversal forced convection. Applied Thermal Engineering 20 (2000) 595-617.

183. P. Grenier, J.J. Guilleminot, F. Meunier, M. Pons. Solar powered solid adsorption cold store. Journal of Solar Energy Engineering 110 (1988) 192-197.

184. M. Pons, J.J. Guilleminot. Experimental data on a solar powered ice maker using activated carbon and methanol adsorption pari. Journal of Solar Energy Engineering 109 (1987) $303-310$

185. D.I. Tchernev. Solar air conditioning and refrigeration systems utilizing zeolites. In: Proceedings of Meetings of Commissions E1-E2. Jerusalem (Israel): International Institute of Refrigeration; 1979. p. 209-15.

186. S. Ülkü. Solar adsorption heat pumps, solar energy utilization: fundamentals and applications. Yüncü H, Paykoc- E, Yener Y, editors. The Netherlands: Martinus Nijkoff Publishers, 1987.

187. S. Ülkü. Adsorption heat pumps. Heat Recovery Systems (1986) 277-284.

188. F. Poyelle, J.J. Guilleminot, F. Meunier. Experimental tests and predictive model of an adsorptive air conditioning unit. Industrial \& Engineering Chemistry Research 38 (1999) 298-309.

189. M. Pons, S. Szarzynski. Accounting for the real properties of the heat transfer fluid in heat-regenerative adsorption cycles for refrigeration. International Journal of Refrigeration 23 (2000) 284-291.

190. G. Restruccia, A. Freni, G. Maggio. A zeolite-coated bed for air conditioning adsorption systems: parametric study of heat and mass transfer by dynamic simulation. Applied Thermal Engineering 22 (2002) 619-630.

191. M. Tatlier, A. Erdem-S-enatalar. Polymeric heat exchangers to increase the COP values of adsorption heat pumps utilizing zeolite coatings. Applied Thermal Engineering 24 (2004) 69-78. 
192. F. Meunier. Second law analysis of a solid adsorption heat pump operating on reversible cascade cycles: application to the zeolite-water pair. Journal of Heat Recovery Systems 5 (1985) 133-141.

193. L. Bonaccorsi, A. Freni, E. Proverbio, G. Restuccia, F. Russo. Zeolite coated copper foams for heat pumping applications. Microporous Mesoporous Materials 91 (2006) 7-14.

194. L. Bonaccorsi, E. Proverbio. Synthesis of thick zeolite 4A coatings on stainless steel. Microporous Mesoporous Materials 74 (2004) 221-229.

195. A. Al-Ansari, H. Ettouney, H. El-Dessouky. Water-zeolite adsorption heat pump combined with single effect evaporation desalination process. Renewable Energy 24 (2001) 91-111.

196. M. Tatlier M, A. Erdem-Senatalar. Optimization of the cycle durations of adsorption heat pumps employing zeolite coatings synthesized on metal supports. Microporous Mesoporous Materials 34 (2000) 23-30.

197. M. Tatlier, A. Erdem-Senatalar. The effects of thermal and mass diffusivities on the performance of adsorption heat pumps employing zeolite synthesized on metal supports. Microporous Mesoporous Materials 28 (1999) 195-203.

198. M. Tatlier, A. Erdem-Senatalar. When do thin zeolite layers and a large void volume in the adsorber limit the performance of adsorption heat pumps? Microporous Mesoporous Materials 54 (2002) 89-96.

199. M. Ramos, R.L. Espinoza, H.J. Horn. Evaluation of a zeolite-water solar adsorption refrigerator. ISES Solar World Congress, Göteborg, Sweden, June 14-19, 2003.

200. M. Tatlier, A. Erdem-Senatalar. The performance analysis of a solar adsorption heat pump utilizing zeolite coatings on metal supports. Chemical Engineering Communications 180 (2000) 169-185.

201. M. Tatlier, A. Erdem-Senatalar. Effects of metal mass on the performance of adsorption heat pumps utilizing zeolite $4 \mathrm{~A}$ coatings synthesized on heat exchanger tubes. International Journal of Refrigeration 23 (2000) 260-268.

202. M. Rothmeyer, M.P. Laxhuber, G. Alefeld. Design and performance of zeolite water heat pumps. In: Proceedings of the 16th international congress of refrigeration in Paris, 1983.

203. M.D. Gross, B. Dawoud. Experimental investigation of and adsorptive heat storage. In: Proceedings of the international sorption heat pump conference, Denver, USA, 2005.

204. D.I. Tchernev, D.T. Emerson. High-efficiency regenerative zeolite heat pump. ASHRAE Transactions 94 (1998) 2024-2032.

205. F. Poyelle, J.J. Guilleminot, F. Meunier. Experimental tests and predictive model of an adsorptive air conditioning unit. Industrial \& Engineering Chemistry Research 38 (1999) 298-309.

206. L.Z. Zhang. Design and testing of an automobile waste heat adsorption cooling system. Applied Thermal Engineering 20 (2000) 103-114.

207. Z. Tamainot-Telto, R.E. Critoph. Advanced solid sorption air conditioning modules using monolithic carbon-ammonia pair. Applied Thermal Engineering 23 (2003) 659-674.

208. K.C. Leong, Y. Liu. Numerical study of a combined heat and mass recovery adsorption cooling cycle. International Journal Heat \& Mass Transfer 47 (2004) 4761-4770.

209. T.F. Qu, R.Z. Wang, W. Wang. Study on heat and mass recovery in adsorption refrigeration cycles. Applied Thermal Engineering 21 (2001) 439-452.

210. A. Aittomäki, M. Härkönen. Zeolite heat pump - adsorption of methanol in synthetic zeolites 13X, A4 and 5A. International Journal of Refrigeration 9 (1986) 240-244.

211. L. Wang, D. Zhu, Y. Tan. Heat transfer enhancement of the adsorber of an adsorption heat pump. Adsorption 5 (1999) 279-286.

212. F.N. Ridha, P.A. Webley. Entropic effects and isosteric heats of nitrogen and carbon dioxide adsorption on chabazite zeolites. Microporous Mesoporous Materials 132 (2010) 22-30

213. Y. Zhong, R.E. Critoph, R. Thorpe. Evaluation of the performance of solid sorption refrigeration systems using carbon dioxide as refrigerant. Applied Thermal Engineering 26 (2006) 1807-1811. 
214. B. Cerkvenik, A. Poredos, F. Ziegler. Influence of adsorption cycle limitations on the system performance. International Journal of Refrigeration 24 (2001) 475-485.

215. L. Wiechmann. Graphite foam with high thermal conductivity and diffusivity conducts heat in all directions. MRS Bull. December 2000.

216. R.Z. Wang, M. Li, X.X. Xu, J.Y. Wu. An energy efficient hybrid system of solar powered water heater and adsorption ice maker. Solar Energy 68 (2000) 189-195.

217. F. Lemmini, F. Meunier. Simulation of an adsorptive solar refrigerator operating in Morocco. Journal of Islamic Academy of Sciences 3 (1990) 273-279.

218. K. Sumathy. An energy efficient solar ice-maker. In: Proceedings of the 8th international symposium on renewable energy education (ISREE8), August 4-8, 2002.

219. M. Pons, J.J. Guilleminot. Design of an experimental solar-powered, solid-adsorption ice maker. Journal of Solar Energy Engineering 108 (1986) 332-337.

220. W. Dechang, W. Jingyi, S. Honggang, W. Ruzhu. Experimental study on the dynamic characteristics of adsorption heat pumps driven by intermittent heat source at heating mode. Applied Thermal Engineering 25 (2005) 927-940.

221. J.Y. Wu, R.Z. Wang, Y.X. Xu. Dynamic analysis of heat recovery process for a continuous heat recovery adsorption heat pump. Energy Conversion \& Management 43 (2002) 2201-2211.

222. M. Li, R.Z. Wang, X.Y. Xu, J.Y. Wu, A.O. Dieng. Experimental study on dynamic performance analysis of a flat-plate solar solid-adsorption refrigeration for ice maker. Renewable Energy 27 (2002) 211-221.

223. M. Li, C.J. Sun, R.Z. Wang, W.D. Ca. Development of no valve solar ice maker. Applied Thermal Engineering 24 (2004) 865-872.

224. S.G. Wang, R.Z. Wang. Recent developments of refrigeration technology in fishing vessels. Renewable Energy 30 (2005) 589-600.

225. R.Z. Wang, J.Y. Wu, Y.X. Xu, W. Wang. Performance researches and improvements on heat regenerative adsorption refrigerator and heat pump. Energy Conversion \& Management 42 (2001) 233-249.

226. R.Z. Wang. Performance improvement of adsorption cooling by heat and mass recovery operation. International Journal of Refrigeration 24 (2001) 602-611.

227. N.M. Khattab. A novel solar-powered adsorption refrigeration module. Applied Thermal Engineering 24 (2004) 2747-2760.

228. N.C. Srivastava, I.W. Eames. A review of adsorbents and adsorbates in solid-vapour adsorption heat pump systems. Applied Thermal Engineering 18 (1998) 707-714.

229. Z. Tamainot-Telto, R.E. Critoph. Adsorption refrigerator using monolithic carbonammonia pair. International Journal of Refrigeration 20 (1997) 146-155.

230. Z. Tamainot-Telto, S.J. Metcalf, R.E. Critoph, Y. Zhong, R. Thorpe. Carbon-ammonia pairs for adsorption refrigeration applications: icemaking, air conditioning and heat pumping. International Journal of Refrigeration 32 (2009) 1212-1229.

231. L.W. Wang, R.Z. Wang, Z.S. Lu, C.J. Chen, K. Wang, J.Y. Wu. The performance of two adsorption ice making test units using activated carbon and a carbon composite as adsorbents. Carbon 44 (2006) 2671-2680.

232. S.J. Metcalf, R.E. Critoph, Z. Tamainot-Telto. Optimal cycles election in carbonammonia adsorption cycles. International Journal of Refrigeration 35 (2012) 571-580.

233. R.E. Critoph, Z. Tamainot-Telto, G.N.L. Davies. Design of an adsorption generator utilising a novel carbon-aluminium laminate. In: Proceedings of the HPC international conference (1997) 349-58.

234. R.E. Critoph. Forced convection adsorption cycle with packed bed heat regeneration. International Journal of Refrigeration 22 (1999) 38-46.

235. A. Elgowainy, S. Shelton, J. Hogan. Experimental investigation of activated carbonammonia heat pump system. In: Proceedings of the international sorption heat pump conference, Denver, USA, (2005).

236. R.E. Critoph. Laboratory testing of an ammonia carbon solar refrigerator. ISES, Solar World Congress, Budapest, Hungary (1993). 
237. D. Miles, S. Shelton. Design and testing of a solid-sorption heat-pump system. Appl Therm Eng 1996;16:389-94.

238. Lu YZ, Wang RZ, Jianzhou S, Xu YX, Wu JY. Practical experiments on an adsorption air conditioner powered by exhausted heat from a diesel locomotive. Appl Therm Eng 2004;24(1051-1059).

239. Wang $\mathrm{LW}$, Wang RZ, $\mathrm{Lu} \mathrm{ZS}, \mathrm{Xu}$ YX, Wu JY. Split heat pipe type compound adsorption ice making unit for fishing boats. Int J Refrig 2006;29:456-68.

240. R.N. Thorpe, R.E. Critoph, Y. Zhong. Sorption of ammonia on calcium chloride/alumina calcium chloride/active carbon composite materials. In: Proceedings of the international sorption heat pump conference, Denver, USA, 2005.

241. Wang WL, Wang ZR, Lu SZ, Xu XY, Wu YJ. Split heat pipe type adsorption ice maker with compound adsorbent: a better choice for fishing boats. In: Proceedings of the international sorption heat pump conference, Denver, USA, 2005.

242. L. Schnabel, H.M. Henning. Experimental and simulation study on the kinetics of water vapour adsorption on different kinds of adsorptive material matrices. In: Proceedings of the international sorption heat pump conference, Denver, USA, 2005.

243. Z.S. Lu, R.Z. Wang, J.Y. Wu, C.J. Chen. Performance analysis of an adsorption refrigerator using activated carbon in a compound adsorbent. Carbon 44 (2006) 747-752.

244. T.X. Li, R.Z. Wang, L.W. Wang, Z.S. Lu, C.J. Chen. Performance study of a high efficient multifunction heat pipe type adsorption ice making system with novel mass and heat recovery processes. International Journal of Thermal Science 46 (2007) 1267-1274.

245. T.X. Li, R.Z. Wang, L.W. Wang, Z.S. Lu, Y.J. Wu. Influence of mass recovery on the performance of a heat pipe type ammonia sorption refrigeration System using $\mathrm{CaCl}_{2} /$ activated carbon as compound adsorbent. Applied Thermal Engineering 28 (2008) 1638-1646.

246. T.X. Li, R.Z. Wang, J.K. Kiplagat, H. Chen, L.W. Wang. A new target-oriented methodology of decreasing the regeneration temperature of solid-gas thermochemical sorption refrigeration system driven by low-grade thermal energy. International Journal of Heat and Mass transfer 54 (2011) 4719-4729.

247. H. Chen-Chia, C. Hsiu-Mei, C. Chien-Hung. Hydrogen adsorption on modified activated carbon. International Journal of Hydrogen Energy 35 (2010) 2777-2780.

248. K. Yoshitsugu, K. Yasuaki, K. Akihiko, S. Nobuaki, H. Tetstuya, H. Tatsumi. Hydrogen adsorption and desorption by carbon materials. Journal of Alloys and Compounds421 (2006) 204-208.

249. S.B. Riffat, M.D. Williams, S. Corr. Adsorption heat pump using HFC refrigerants. Int Journal of Energy Research 21 (1997) 481-494.

250. M. Pons, F. Meunier, G. Cacciola, R.E. Critoph, M. Groll, L. Puigjaner . Thermodynamic based comparison of sorption systems for cooling and heat pumping. International Journal of Refrigeration 22 (1999) 5-17.

251. J. Hu Eric. A study of thermal decomposition of methanol in solar powered adsorption refrigeration systems. Solar Energy 52 (1998):325-329.

252. I.I. El-Sharkawy, M. Hassan, B.B. Saha, KoyamaS., M.M. Nasr. Study on adsorption of methanol onto carbon based adsorbents. International Journal of Refrigeration 32 (2009) $1579-1586$.

253. L.W. Wang, J.Y. Wu, R.Z. Wang, Y.X. Xu, S.G. Wang, X.R. Li. Study of the performance of activated carbon-methanol adsorption systems concerning heat and mass transfer. Applied Thermal Engineering 23 (2003)1605-1617.

254. Y. Hamamoto, K.C.A. Alam, B.B. Saha, S. Koyama, A. Akisawa, T. Kashiwagi. Study on adsorption refrigeration cycle utilizing activated carbon fibers. Part 1. Adsorption characteristics. International Journal ofRefrigeration 29 (2006) 305-314.

255. H.T. Chua, K.C. Ng, A. Malek, T. Kashiwagi, A. Akisawa, B.B. Saha . Multi-bed regenerative adsorption chiller-improving the utilization of waste heat and reducing the chilled water outlet temperature fluctuation. International Journal ofRefrigeration 24 (2001) 124-136. 
256. B.B. Saha El-Sharkawy, S. Koyama, J. He, K.C. Ng, C. Yap. Experimental investigation on activated carbon-ethanol pair for solar powered adsorption cooling applications. International Journal of Refrigeration 31(2008)1407-1413.

257. B.B. Saha, I.I. El-Sharkawy, A. Chakraborty, S. Koyama. Study on an activated carbon fiber-ethanol adsorption chiller: part I e System description and modeling. International Journal of Refrigeration 30 (2007) 86-95.

258. C. Shen, A. Grande Carlos, P. Li, J. Yu, A.E. Rodrigues. Adsorption equilibria and kinetics of $\mathrm{CO}_{2}$ and $\mathrm{N}_{2}$ on activated carbon beads. Chemical EngineeringJournal 160 (2010) 398-407.

259. R.G. Oliveira, V. Silveira Jr, R.Z. Wang. Experimental study of mass recovery adsorption cycles for ice making at low generation temperature. Applied Thermal Engineering26 (2006) 303-311.

260. G. Restuccia, A. Freni, G. Cacciola. Adsorption beds of zeolite on aluminium sheets. In: Proceedings of International Sorption Heat Pump Conference, Munich, (1999).

261. L. Marletta, G. Maggio, A. Freni, M. Ingrasciotta, G. Restuccia. A non-uniform temperature non-uniform pressure dynamic model of heat and mass transfer in compact adsorbent beds. International Journal of Heat and Mass transfer 45 (2002) 3321-3330.

262. B. Dawoud, T. Miltkau. Dynamic modeling of the combined heat and mass transfer during the adsorption/desorption of water vapor into/from a zeolite layer of an adsorption heat pump. International Journal of Thermal Sciences41 (2002) 753-762.

263. M. Kanamori, M. Hiramatsu, K. Katsurayama, F. Watanabe, M. Hasatani. Production of cold heat energy by alcohol/activated carbon adsorption heat pump with a disk-module-type adsorber. Journal of Chemical Engineering of Japan 30 (1997) 434-439.

264. L.W. Wang, J. Wu, R.Z. Wang, Y. Wu. Influences of heat and mass transfer characteristics on the performance of adsorption ice maker driven by waste heat. In: Proceedings of International Sorption Heat Pump Conference, ISHPC 02, Shanghai, (2002) 650-654.

265. Y.B. Gui,, R.Z. Wang, W. Wang, J.Y Wu, Y.X Xu. Performance modelling and testing on a heat-regenerative adsorptive reversible heat pump. Applied Thermal Engineering 22 (2002) 309-320.

266. A Freni, M.M Tokarev, G Restuccia, A.G Okunev, Y.I Aristov . Thermal conductivity of selective water sorbents under the working conditions of a sorption chiller. Applied Thermal Engineering 22 (2002) 1631-1642.

267. G. Restuccia, S. Vasta, A. Freni, F. Russo, I.Y. Aristov. An advanced solid sorption chiller using SWS-1L: performance analysis and hydrothermal cycling stability of the sorbent bed. In: Proceedings of the international sorption heat pump conference, Denver, USA, (2005).

268. B. Dawoud. A hybrid solar-assisted adsorption cooling unit for vaccine storage. In: Proceedings of the international sorption heat pump conference, Denver, USA, (2005).

269. R.E. Critoph, L. Turner. Heat transfer in granular activated carbon beds in the presence of adsorbable gases. International Journal of Heat Mass Transfer 38 (1995), 1577-1585.

270. T.H. Eun, H.K. Songa, J. H. Hana, K.H. Lee. Enhancement of heat and mass transfer in silica-expanded graphite composite blocs for adsorption heat pumps; Part I. Characterisation of the composite blocs. International Journal of Refrigeration 23 (2000) 64-73.

271. T.H. Eun, H.K. Song, J.H. Han, K.H. Lee, J.N. Kim. Enhancement of heat and mass transfer in silica-expanded graphite composite blocks for adsorption heat pumps. Part II. Cooling system using the composite blocks. International Journal of Refrigeration 23 (2000) $74-81$.

272. R.G. Oliveira, R.Z. Wang, C. Wang. Evaluation of the cooling performance of a consolidated expanded graphite-calcium chloride reactive bed for chemisorption icemaker. International Journal of Refrigeration 30 (2007) 103-112.

273. J.J. Guilleminot, J.B. Chalfen, A. Choisier. Heat and mass transfer characteristics of composites for adsorption heat pumps. In: International Absorption Heat Pump Conference, AES 31, ASME, New York, (1993). 
274. M. Pons, D. Laurent, F. Meunier. Experimental temperature fronts for adsorptive heat pump applications. Applied Thermal Engineering 16 (1996) 395-404.

275. M.A. Lambert. Design of solar powered adsorption heat pump with ice storage. Applied Thermal Engineering 27 (2007) 1612-1628.

276. J.S. Metcalf. Simulation of the effect of generator heat transfer parameters on power density and efficiency in multiple-bed regenerative carbon-ammonia sorption heat pumps. In: Proceedings of the international sorption heat pump conference, Denver, USA, (2005).

277. T.Z. Telto. Compact sorption generator prototype. In: Proceedings of the international sorption heat pump conference, Denver, USA, (2005).

278. R.E. Critoph. Forced convection adsorption cycles. Applied Thermal Engineering 18 (1998) 799-807.

279. Y.B. Gui, R.Z. Wang, W. Wang, J.Y. Wu, Y.X. Xu. Performance modeling and testing on a heat-regenerative adsorptive reversible heat pump. Applied Thermal Engineering 22 (2002) 309-320.

280. Y.U. Wu, R.Z. Wang, Y.X. Xu. Influence of adsorption and desorption capacity on operating process for adsorption heat pump. Applied Thermal Engineering 22 (2002) 471476.

281. A.S. Ülkü, M. Mobedi. Adsorption in energy storage. proceedings NATO Adv Stud Inst Energy Storage Syst Ser E Appl Sci 167 (1989) 487-507.

282. B.B. Saha, S. Koyama, I.I. El-Sharkawy II, Kuwahara K, Kariya K, Ng KC. Experiments for measuring adsorption characteristics of an activated carbon fiber/ethanol pair using a plate-fin heat exchanger. HVAC\&R Research 12 (2006) 767-782.

283. Z. Tamainot-Telco, R.E. Critoph. Solar sorption refrigerator using a CPC collector. Renewable Energy 16 (1999) 735-738.

284. R.E. Critoph, Z. Tamainot-Telto. Thermophysical properties of monolític carbon. International Journal of Heat and Mass Transfer 43 (2000), 2053-2058.

285. S.G. Wang, R.Z. Wang, J.Y. Wu, Y.X. Xu. Experimental results and analysis for adsorption ice-making system with consolidated adsorbent. Adsorption 9 (2003) 349-358.

286. R.E.Critoph, Z. Tamainot-Telto, G.N.L. Davies. A prototype of a fast cycle adsorption refrigerator utilising a carbon-aluminium laminate. Procedings Instn Mech Engrs, Part A: Journal of Power and Energy 214 (2000) 439-448.

287. S.K. Gordeev. Novel carbide and carbide materials with high thermal conductivity and high porosity as candidates for Applications in heat pipes and heat pumps. In: Proceedings of V Minsk International Seminar, Heat Pipes, Heat Pumps and Refrigerators, Belarus 6374 (2003).

288. C. Rivera, I. Pilatowsky, E. Méndez, W. Rivera. Experimental study of a thermoschemical refrigerator using the barium chloride-ammonia reaction. International Journal of Hydrogen Energy 32 (2007) 3154-3158.

289. C. Duenas, I. Pilatowskya, R.J. Romero, A. Oskama, A.P. Finck. Dynamic study of the thermal behavior of solar thermochemical refrigerator: barium chloride-ammonia for ice production. Solar Energy Materials and Solar Cells 70 (2001) 401-413.

290. K.J. Kim, K.T. Feldman Jr, G. Lloydt, A. Razanit. Compressor-driven metal-hydride heat pumps. Applied Thermal Engineering 17 (1997) 551-560.

291. E. Willers, M. Groll. Evaluation of metal hydride machines for heat pumping and cooling applications. International Journal of Refrigeration 22 (1999) 47-58.

292. B. Sakintuna, F. Lamari-Darkrim, M. Hirscher. Metal hydride materials for solid hydrogen storage: a review. International Journal of Hydrogen Energy 32 (2008) 11211140.

293. E. Willers, M. Groll. Evaluation of metal hydride machines for heat pumping and cooling applications. International Journal of Refrigeration 22 (1999) 47-58.

294. J. Ni, H. Liu. Experimental research on refrigeration characteristics of a metal hydride heat pump in auto air-conditioning. International Journal of Hydrogen Energy 32 (2007) 2567-2572. 
295. A.S. Chernikov, L.A. Izhvanov, A.L. Solovey, V.P. Frolov, S. Yul. An installation for water cooling based on a metal hydride heat pump. Journal of alloys and compounds 13301332 (2002) 907-910.

296. R. Gopal, S. Murthy. Experiments on a metal hydride cooling system working with $\mathrm{ZrMnFe} / \mathrm{MmNi4} .5 \mathrm{Al} 10.5$ pair. International Journal of Refrigeration 22 (1999) 137-149.

297. Kato Y, Yamashita Norimichi, Kobayashi Kei, Yoshizawa Yoshio. Kinetic study of the hydrogen of magnesium oxide for a chemical heat pump. Applied Thermal Engineering 1996;16(I1):853-62.

298. J.V. Veselovskaya, M.M. Tokarev, Y. Aristov. Novel ammonia sorbents porous matrix modified by active salt for adsorptive heat transformation 1. Barium chloride in various matrices. Applied Thermal Engineering 30 (2010) 584-589.

299. J.V. Veselovskaya, R.E. Critoph, R.N. Thorpe, S. Metcalf, M.M. Tokarev, Y. Aristov. Novel ammonia sorbents porous matrix modified by actives alt for adsorptive heat transformation: 3 . Testing of $\mathrm{BaCl}_{2} /$ vermiculite composite in a lab-scale adsorption chiller. Applied Thermal Engineering 30 (2010) 1188-1192.

300. D. Grekova Alexandra, J.V. Veselovskaya, M.M. Tokarev, L.G. Gordeeva. Novel ammonia sorbents porous matrix modified by active salt for adsorptive heat transformation: 5. Designing the composite adsorbent for ice makers. Applied Thermal Engineering 37 (2012) 80-86.

301. J. Ehrenmann, S. K. Henninger, C. Janiak. Water Adsorption Characteristics of MIL101 for Heat-Transformation Applications of MOFs European Journal of Inorganic. Chemistry (2011) 471-474.

302. P. Küsgens, M. Rose, I. Senkovska, H. Fröde, A. Henschel, S. Siegle, S. Kaskel, Characterization of metal-organic frameworks by water adsorption. Microporous Mesoporous Materials, 120 (2009) 325-330.

303. G. Akiyama, R. Matsuda, S. Kitagawa, Highly Porous and Stable ... as Water Sorption Materials. Chemistry Letters 39 (2010) 360-361

304. S. Henninger, F. Schmidt, H.-M. Henning, Novel Sorption Materials for Solar Heating and Cooling. Adsorption 17 (2011), 833-843.

305. K. Wang, J.Y. Wu, Z.Z. Xia, S.L. Li, R.Z. Wang. Design and performance prediction of a novel double heat pipes type adsorption chiller for fishing boats. Renewable Energy 33 (2008) 780-790.

306. S.L. Li, Z.Z. Xia, J.Y. Wu, J. Li, R.Z. Wang, L.W. Wang. Experimental study of a novel $\mathrm{CaCl}_{2} /$ expanded graphite $/ \mathrm{NH}_{3}$ adsorption refrigerator. International Journal of Refrigeration 33 (2010) 61-69.

307. P. Neveu, J. Castaing. Solid-gas chemical heat pumps: field of application and performance of the internal heat recovery. Heat RecovERY Systems CHP 13 (1993) 233251.

308. M. Sorin, B. Spinner, D. Stitou. Thermodynamic techniques for the conceptual design of thermochemical refrigerators using two salt materials. Chemistry Engineering Science 57 (2002) 4243-4251.

309. R.G. Oliveira, R.Z. Wang, T.X. Li. Transient analysis of a chemisorption air conditioning system operating under different kinds of cycle. Industrial Engineering Chemistry Research 47 (2008) 1102-1110.

310. T.X. Li, R.Z. Wang, J.K. Kiplagat, L.W. Wang, R.G. Oliveira. A conceptual design and performance analysis of a triple-effect solidegas thermochemical sorption refrigeration system with internal heat recovery. Chemistry Engineering Science 64 (2009) 3376-3384.

311. T.X. Li, R.Z. Wang, R.G. Oliveira, J.K. Kiplagat, L.W. Wang. A combined double-way chemisorption refrigeration cycle based on adsorption and resorption processes. International Journal of Refrigeration 32 (2009) 47-57.

312. Li TX, Wang RZ, Kiplagat JK, Wang LW. Performance improvement of a combined double-way sorption refrigeration cycle using re-heating process. AIChE J 2010;56:477-84.

313. T.X. Li, R.Z. Wang, J.K. Kiplagat, L.W. Wang. Experimental study on a combined double-way chemisorption refrigeration system. International Journal of Refrigeration 34 (2011) 914-921. 
314. Li TX, Wang RZ, R.G. Oliveira, L.W. Wang. Performance analysis of an innovative multi-mode, multi-salt and multi-effect chemisorption refrigeration system. AIChE Journal 53 (2007) 3222-3230.

315. L. Xu, R.Z. Wang, T.X. Li, L.W. Wang. Experimental study on the performance of double-effect and double-way thermochemical sorption refrigeration cycle. Applied Thermal Engineering 31 (2011) 3658-3663.

316. J. Berthiaud J. Procédés à sorption solide/gaz pour le transport de chaleur et de froid à longue distance [Doctor dissertation]. Perpignan, France: PROMES (2007).

317. T.X. Li, R.Z. Wang, J.K. Kiplagat. A target-oriented solid-gas thermochemical sorption heat transformer for integrated energy storage and energy upgrade. AIChE Journal 59 (2013) 1334-1347.

318. D.M. Ruthven. Principles of adsorption and adsorption processes. New York: Wiley; (1984).

319. D.D. Do. Adsorption analysis: equilibria and kinetics. London: Imperial College Press; (1998).

320. V. Ponec, Z. Knor, S. Cerny. Adsorption on solids. London: Butterworths Group, (1974).

321. J. Oscik, I.L. Cooper. Adsorption. Chichester: Ellis Horwood Limited, (1982).

322. S. Kaskel. Special Issue: Chemieparks - Poröse Materialien, Teil 2. Chemistry Ingeniur TEchnik 82 (2010) 1019-1023.

323. M. Thommes, Physical Adsorption Characterization of Nanoporous Materials. Chemistry Ingeniur TEchnik 82 (2010) 1059-1073.

324. D. Tanaka, K. Nakagawa, M. Higuchi, S. Horike, Y. Kubota, T. C. Kobayashi, M. Takata, S. Kitagawa. g Process in Flexible Porous Coordination Polymer. Angewandte Chemie. 2008, 120, 3978.

325. S. K. Henninger, G. Munz, K.-F. Ratzsch, P. Schossig. Cycle stability of sorption materials and composites for the use in heat pumps and cooling machines. Renewable Energy 36 (2011) 3043-3049.

326. M.S. Fernandes, G.J. V.N. Brites, J.J. Costa, A.R. Gaspar, V.A.F. Costa. Review and future trends of solar adsorption refrigeration systems. Renewable and Sustainable Energy Reviews 39 (2014) 102-123.

327. K. E. N'Tsoukpoe, H. Liu, N. Le Pierrès, L.Luo. A review on long-term sorption energy storage. Renewable and Sustainable Energy Reviews 13 (2009) 2385-2396.

328. T. Schmidt, D. Mangold, H. Müller-Steinhagen. Central solar heating plants with seasonal storage in Germany. Solar Energy 76 (2004) 165-74

329. A. Solé, I. Martorell, L. F. Cabeza. State of the art on gas-solid thermochemical energy storage systems and reactors for building applications. Renewable and Sustainable Energy Reviews 47 (2015) 386-398

330. J. C. Hadorn. Thermal energy storage for solar and low energy buildings: state of the art by the IEA solar heating and cooling task, 32. 2005.

331. J. Heier, C. Bales, V. Martin. Combining thermal energy storage with buildings - a review. Renewable and Sustainable Energy Reviews 42 (2015) 1305-1325.

332. B. Michel, P. Neveu, N. Mazet. Comparison of closed and open thermochemical processes for long-term thermal energy storage applications. Energy 72 (2014) 702-716.

333. D. Aydin, S.P. Casey, S. Riffat. The latest advancements on thermochemical heat storage systems. Renewable and Sustainable Energy Reviews 41 (2015) 356-367.

334. K.E. N'Tsoukpoe, G. Restuccia, T. Schmidt, X. Py. The size of sorbents in low pressure sorption or thermochemical energy storage processes. Energy 77 (2014) 983-998.

335. A. H. Abedin, M. A. Rosen. Closed and open thermochemical energy storage: Energyand exergy- based comparisons. Energy 41 (2012) 83-92.

336. H. A. Zondag, A. Kalbasenka, M. van Essen, L. Bleijendaal, R. Schuitema, W. van Helden, L. Krosse. First studies in reactor concepts for thermochemical storage. 2009. Available from: ftp://ftp.ecn.nl/pub/www/library/report/ 2009/m09008.pdf (last accessed 21/05/15). 
337. A. Hauer. Thermal energy storage with zeolite for heating and cooling applications.In: Proceedings of 3rd workshop of annex 17 ECES IA/IEA. 2002

338. B. Mette, H. Kerskes, H. Drück. Concepts of long-term thermochemical energy storage for solar thermal applications - Selected examples. Energy Procedia 30 (2012) 321-330

339. C. Bales . Laboratory Tests of Chemical Reactions and Prototype Sorption Storage Units: A Report of IEA Solar Heating and Cooling programme - Task 32 Advanced storage concepts for solar and low energy buildings.

340. H.A. Zondag, V.M. v Essen, L.P.J. Bleijendaal, B.W.J. Kikkert, M. Bakker. Economical feasibility of sorption heat storage. In: Proceedings of the 5th International Renewable Energy Storage Conference IRES 2010, Berlin, Germany, 2010.

341. J. Cot-Gores, A. Castell, L. F. CAbeza. Thermochemical energy storage and conversión: A-state-of-the-art review of the experimental research practical conditions. Renewable and Sustainable Energy Reviews 16 (2012) 5207-5224.

342. W. Wongsuwan. Performance analysis of reactor for thermochemical energy storage (TCES). Final report. Department of Mechanical Engineering, Faculty of Engineering, Chiang Mai University, Thailand, 2004.

343. K. Iammak, W. Wongsuwan, T. Kiatsiriroj. Investigation of modular chemical energy storage performance. In: The joint international conference on "sustainable energy and environment (SEE)", 2004

344. H. Lahmidi, S. Mauran, V. Goetz. Definition, tests and simulation of a thermochemical storage process adapted to solar thermal system. Solar Energy 80 (2009):883-893.

345. W. v. Helden, M. Yamaha, C. Rathgeber, A. Hauer, F. Huaylla, N. Le Pierrès, B. Stutz, B. Mette, P. Dolado, A. Lazaro, J. Mazo, M. Dannemand, S. Furbo, A.Campos-Celador, Gonzalo Diarce, R. Cuypers, A. König-Haagen, S. Höhlein, Dieter Brüggemann, B. Fumey, R. Weber, R. Köll, W. Wagner, X. Daguenet-Frick, P. Gantenbein, F. Kuznik. Applications of Compact Thermal Energy Storage. IEA SHC Task 42/ECES Annex 29 - Working Group B. Energy Procedia in press. SHC 2015, International Conference on Solar Heating and Cooling for Buildings and Industry

346. L. Jiang, F.Q. Zhu, L.W. Wang, C.Z. Liu, R.Z. Wang. Experimental investigation on a MnCl2-CaCl2-NH3 thermal energy storage system. Renewable Energy 91 (2016) 130-136.

347. www.merits.eu (last access 21/04/16)

348. T. Yan, R.Z. Wang, T.X. Li, L.W. Wang, I.T. Fred. A review of promising candidate reactions for chemical heat storage. Renewable and Sustainable Energy Reviews 43 (2015) 13-31.

349. T.X. Li, R.Z. Wang, J.K. Kiplagat, Y.T. Kang. Performance analysis of an integrated energy storage and energy upgrade thermochemical solid-gas sorption system for seasonal storage of solar thermal energy. Energy 50 (2013);:454-67

350. R.V.D. Weber. Long-term heat storage with NaOH. Vacuum 2008;82(7):708-16.

351. C. Bales, S. Nordlander TCA evaluation - lab measurements, modelling and system simulations. Borlänge, Sweden: Högskolan Dalarna, 2005

352. Quinnell JA, Davidson JH, Burch J. Liquid Calcium Chloride Solar Storage: Concept and Analysis. J SOL ENERG-T ASME 2011;133:-.

353. Tanaka T, Sakuta K, Kamimoto M, Tani T, Sawata S, Horigome T. Solar thermal energy storage using heat of dilution: analysis of heat generation in multi-stage mixing column. Energy Convers 1978;18:57-65.

354. Wyman C, Castle J, Kreith F. A review of collector and energy storage technology for intermediate temperature applications. Sol Energy 1980;24: 517-40.

355. A. J. Carrillo, J. Moya, A. Bayón, P. Jana, V. A. de la Peña O’Shea, M. Romero, J. González-Aguilar, D. P. Serrano, P. Pizarro, J. M. Coronado. Thermochemical energy storage at high temperatura via redox cyles of Mn and Co oxides: Pure oxides versus ones. Solar Energy Materials \& Solar Cells 123 (2014) 47-57.

356. V. M. Van Essen, J. C. Gores, L. P. J. Bleijendaal, H. A. Zondag, R. Schuitema, M. Bakker, W. G. J. van Helden. Characterization of salt hydrates for compact seasonal 
thermochemical storage. In: Third International conference of energy sustainability 2009.

San Francisco (USA); 19-23 July 2009

357. A. Frazzica, A. Sapienza, A. Freni. Adsorption heat storage: state of the art and future perspectives. $16^{\text {th }}$ CIRIAF Nationa Congress 2016, April 7-9, 2016, Assisi, Italy.

358. A. Solé, L. Miró, Camila Barreneche, I. Martorell, L. F. Cabeza. Corrosion of metals and salt hydrates used for thermochemical energy storage. Renewable Energy 75 (2015) 519-523. http://dx.doi.org/10.1016/j.renene.2014.09.059

359. A. Solé, C. Barreneche, I. Martorell, L. F. Cabeza. Corrosion evaluation and prevention of reactor materials to contain thermochemical materials for thermal energy storage. Applied Thermal Engineering 94 (2016) 355-363.

http://dx.doi.org/10.1016/j.applthermaleng.2015.10.156

360. F. Meunier. Solid sorption: an alternative to CFCs. Heat Recovery Systems and CHP 13 (1993) 289-295.

361. K. E. N'Tsoukpoe, G. Restuccia, T. Scmidt, X. Py. The size of sorbents in low pressure sorption or thermochemical energy storage processes. Energy 77 (2014) 983-998.

362. B.Q. Han, H.Y. Yuan, D.Q. Yang, G.X. Liu. Utilization of natural zeolites for solar energy storag, (1994).

363. M. Mobedi, G.G. Ilis, S. Ülkü. Comparison of uniform and non-uniform pressure approaches used to analyze an adsorption process in a closed type adsorbent bed. Transp Porous Med 98 (2013) 81-101.

364. K.E. N'Tsoukpoe, T. Schmidt, H. U. Rammelberg, B. A. Watts, W. K. L. Ruck. A systematic multi-step screening of numerous salt hydrates for low temperature thermochemical energy storage. Applied Energy 124 (2014) 1-16.

365. Van Essen VM, Cot Gores J, Bleijendaal LPJ, Zondag HA, Schuitema R, Bakker M, et al. Characterization of salt hydrates for compact seasonal thermochemical storage. In: Proceedings of Proceedings of the ASME 3rd International Conference on Energy Sustainability 2009 San Francisco, USA, (2009).

366. F. Bertsch, B. Mette, S. Asenbeck, H. Kerskes, H. Müller-Steinhagen. Low temperature chemical heat storage - an investigation of hydration reactions; 2009. Available from: https://talon.stockton.edu/eyos/energy studies/content/docs/effstock09/Session_6_2 Therm ochemical storage/49.pdf (last access 21/04/16)

367. K. Posern, C. Kaps. Calorimetric studies of thermochemical heat storage materials based on mixtures of $\mathrm{MgSO}_{4}$ and $\mathrm{MgCl}_{2}$. Thermochimica Acta 502 (2010) 73-76.

368. S. Mauran, P. Parades, F. L'haridon, Heat and mass transfer in consolidated reacting beds for thermochemical systems, Heat Recovery system CHP 13 (1993) 315-319.

369. L.G. Gordeeva and Yu. I. Aristov. Composites 'salt inside porous matrix' for adsorption heat transformation: a current state-of-the-art and new trends. International Journal of LowCarbon Technologies 7 (2012) 288-302.

370. S. Hongois, P. Stevens, A.S. Coince, F. Kuznik, J.J. Roux. Thermochemical storage using composite materials. In: Proceedings of the Eurosun 2008, 1st international conference on solar heating, cooling and buildings, (2008).

371. I. Fujiwara, M. Sato, M. Hiramatsu, Y. Furusawa, H. Satake, M. Ebe. Equilibrium of NH4SCN-NH3 system for thermal energy storage. Energy Convers Manage 27 (1987) $159-162$.

372. D. Mugnier, V. Goetz. Energy storage comparison of sorption systems for cooling and refrigeration. Solar Energy 71 (2001) 47-55.

373. G. Li, Y. Hwang, R. Radermacher, H-H. Chun. Review of cold storage materials for subzero application. Energy 51 (2013) 1-17

374. K. Korhammer, M-M Druske, A. Fopah-Lele, H. U. Rammelberg, N. Wegscheider, O. Opel, T. Osterland, W. Ruck. Sorption and thermal characterization of composite materials based on chlorides for thermal energy storage. Applied Energy 162 (2016) 1462-1472.

375. P. Tatsidjodoung, N. Le Pierrès, L. Luo. A review of potential materials for thermal energy storage in building applications. Renewable and Sustainable Energy Reviews 18 (2013) 327-349. 